\title{
Dynamic aspect of the chiral phase transition in the mode coupling theory
}

\author{
K. Ohnishi ${ }^{\mathrm{a}, 1}$, K. Fukushima ${ }^{\mathrm{b}, \mathrm{c}}$ and K. Ohta $^{\mathrm{a}}$ \\ a Institute of Physics, University of Tokyo, \\ 3-8-1 Komaba, Meguro-ku, Tokyo 153-8902, Japan \\ ${ }^{\mathrm{b}}$ Center for Theoretical Physics, Massachusetts Institute of Technology, \\ 77 Massachusetts Avenue, Cambridge, MA 02139, U.S.A. \\ ${ }^{\mathrm{c}}$ Department of Physics, University of Tokyo, \\ 7-3-1 Hongo, Bunkyo-ku, Tokyo 113-0033, Japan
}

\begin{abstract}
We analyze the dynamic aspect of the chiral phase transition. We apply the mode coupling theory to the linear sigma model and derive the kinetic equation for the chiral phase transition. We challenge Hohenberg and Halperin's classification scheme of dynamic critical phenomena in which the dynamic universality class of the chiral phase transition has been identified with that of the antiferromagnet. We point out a crucial difference between the chiral dynamics and the antiferromagnet system. We also calculate the dynamic critical exponent for the chiral phase transition. Our result is $z=1-\eta / 2 \cong 0.98$ which is contrasted with $z=d / 2=1.5$ of the antiferromagnet.
\end{abstract}

Key words: Chiral phase transition, Dynamic critical phenomena, Slow mode, Mode coupling theory, Critical exponent

PACS: 05.20.Dd, 11.10.Wx, 11.30.Rd

Email addresses: konishi@nt1.c.u-tokyo.ac.jp (K. Ohnishi), kenji@lns.mit.edu (K. Fukushima), ohta@nt1.c.u-tokyo.ac.jp (K. Ohta).

1 Present address: Yukawa Institute for Theoretical Physics, Kyoto University, Kyoto 606-8502, Japan

Preprint submitted to Elsevier Science 21 January 2018 


\section{Introduction}

Among the central issues in the study of QCD (Quantum ChromoDynamics), there exists the problem of the spontaneous chiral symmetry breaking and its restoration, i.e., the chiral phase transition. It is believed that the chiral phase transition occurs under an extreme condition like high temperature or high density. The critical dynamics of the second order chiral phase transition for the two flavor massless QCD is not only of theoretical interest but also of importance in understanding the relativistic heavy ion collision experiments. The critical phenomena significantly affect how hot matter cools down. There are a lot of works aimed to look into the static (equilibrium) nature of the chiral phase transition, including the lattice QCD simulation $[1,2,3,4,5]$. Our objective in the present paper is to investigate the dynamic (non-equilibrium or real-time) property of the second order chiral phase transition $[6,7,8,9,10,11,12,13]$. Knowledge of the dynamic critical behavior would be indispensable to understand the relativistic heavy ion collision experiments because the system evolves essentially through non-equilibrium states. Anomalous dynamic critical phenomena such as the critical slowing down and the softening of propagating modes have significant relevance to the matter going toward or coming from a quark-gluon plasma. Throughout this paper, we will restrict ourselves to the chiral phase transition at zero baryon number density.

In general, whenever we consider a critical point of second order, it is useful to classify the critical point according to the universality class. The critical points in the same universality class are to show exactly the same critical behavior unless there appears another fixed point or the interaction is infinitely long-ranged. For the static case, as is well known, the universality class is determined solely by the global symmetry of the system. The systems having the same symmetry belong to the same static universality class. On the other hand, the dynamic universality class is more complicated to classify. A classification method, which we will briefly review in the next section, has been proposed and established by Hohenberg and Halperin. Actually, all the observed critical points, such as the liquid-gas transition, transitions in a ferromagnet, in an antiferromagnet and in a superfluid, have been classified systematically and comprehensively in the review article by Hohenberg and Halperin [14].

Consideration on the universality class of the chiral phase transition has already been given. The static universality class of the two-flavor chiral phase transition is identified with that of the ferromagnet and antiferromagnet because they all have the rotational $\mathrm{O}(4)$ symmetry [1]. The dynamic universality class of the chiral phase transition has also been discussed by Rajagopal and Wilczek [7]. They argue that the chiral phase transition belongs to the same dynamic universality class as that of the antiferromagnet. Certainly it is concluded that the dynamic universality classes of the two systems would be 
identical according to the classification method of Hohenberg and Halperin. Since the nature of the antiferromagnet has been analyzed and understood enough, the identification of the dynamic universality class means that the nature of the chiral phase transition is understood as well. There does exist, however, a crucial difference between the two systems in the appearance of physical modes, as pointed out in Ref. [10]. The difference is so crucial that the dynamic universality classes of the two systems cannot be regarded as identical any longer and Hohenberg and Halperin's classification method itself must be reexamined carefully. In the next section, we will take a close look at the two systems to reveal the difference.

Once the two systems are realized to be distinctive, it is necessary to reanalyze the dynamic aspect of the chiral phase transition without resorting to Hohenberg and Halperin's classification method. We will adopt the mode coupling theory $[15,16,17,18]$ in order to disclose the physical modes and write down the kinetic equations in a chiral effective model.

The plan of this paper is as follows. In Sec. 2, we will give a general review on the dynamic critical phenomena and point out the difference between the antiferromagnet and the chiral phase transition. In Sec. 3, we will review the mode coupling theory briefly. In Sec. 4, we will apply the mode coupling theory to the $\mathrm{O}(2)$ linear sigma model in order to see what kinds of slow modes appear near the phase transition. In Sec. 5 we will derive the kinetic equation in the $\mathrm{O}(4)$ linear sigma model and we will calculate the dynamic critical exponents in Sec. 6. The exponent will be found to be $z=1-\eta / 2 \cong 0.98$, which is contrasted with $z=d / 2$ of the antiferromagnet. Finally, we will give the summary in Sec. 7.

\section{Dynamic universality class of the chiral phase transition}

In this section, we will discuss the dynamic universality class of the chiral phase transition with emphasis on the difference from the antiferromagnet. For that purpose, it is inevitably necessary to explain the notion of the slow mode. In Subsec. 2.1, we will give a general review on the dynamic critical phenomena, in which we will explain the slow variable, the slow mode and the classification method of the dynamic universality class by Hohenberg and Halperin. In Subsec. 2.2, we will discuss the chiral phase transition. 


\subsection{Dynamic critical phenomena}

One of the prominent ingredients of the dynamic critical phenomena would be the critical slowing down, that is, the long (and infinite) relaxation time near (and at) a critical point. The critical slowing down is attributed to the slow motion of the long wavelength fluctuations of the slow variables. The slow variables near a critical point consist of the order parameter and the conserved quantities involved in the system. The slowness of the order parameter fluctuations can be understood by considering a flat thermodynamic potential with respect to the order parameter near the critical point, which indicates a weak restoring force on the order parameter fluctuations. Alternatively we can imagine the correlated domains of the order parameter to understand the critical slowing down. Near the critical point, the size of the domains, i.e., the correlation length $\xi$, becomes so large that the motion of the domains gets very dull and slow. Since $\xi$ diverges just at the critical point, the slowness of the motion becomes infinite, leading to the divergent relaxation time.

The motion of the long wavelength fluctuation of a conserved quantity is kinematically slow even away from the critical point. In fact, the fluctuation of a conserved quantity with zero wavenumber, $\vec{q}=0$, has an infinite relaxation time because of conservation of the quantity. [Note that $\partial_{\mu} j^{\mu}=0$ means $j^{0}$ is time independent when $\vec{q}=0$.] The fluctuation with a small but finite wavenumber has a long but finite relaxation time. Near the critical point, it is known that the coupling between the order parameter and the conserved quantities becomes very strong. Through the coupling, the fluctuations of the conserved quantity even with a finite wavenumber come to have an infinite relaxation time at the critical point.

We have seen the slowness of the long wavelength fluctuation of the slow variables. The relaxation time for those fluctuations is so long and amounts to the macroscopic scale belonging to the kinetic regime. On the other hand, for the degrees of freedom other than the long wavelength fluctuation of the slow variables, namely the short wavelength fluctuations of the slow variables and the fluctuations of the coordinates other than the slow variables, the motions remain rapid near the critical point. Consider a non-equilibrium system near the critical point after a macroscopic time has elapsed. The non-equilibrium fluctuations of the rapid degrees of freedom will have already relaxed to constitute the heat bath, while the long wavelength fluctuations of the slow variables will still remain as non-equilibrium fluctuations. Thus, by tracing the time evolution of the long wavelength fluctuations of the slow variables, we can make the sufficient and even complete description of non-equilibrium processes in the kinetic regime. The mode coupling theory developed by Kawasaki $[15,16,17,18]$ is one of the schemes which provides us with the equation of motion for the critical fluctuations, that is, the kinetic equation near the critical point. 
The long wavelength fluctuations of the slow variables or their linear combinations constitute what is called the slow modes. The slow modes appear as narrow peaks in the spectral functions $N(\omega, \vec{q})$ associated with the slow variables. There are two kinds of slow modes; a diffusive mode and a propagating mode. The diffusive mode is a mode that is purely dissipative, while the propagating mode involves an oscillation besides a dissipation. Examples for the propagating mode are the sound wave, the spin wave and the "particle mode." For definiteness, consider the critical fluctuation with a fixed wavenumber $\vec{q} \ll 1[\vec{q}$ not necessarily vanishing] in the following explanation. The diffusive mode corresponds to the pole of the spectral function in the complex plane of the frequency $\omega$ having only an imaginary part $\Gamma$. In the spectrum as a function of $\omega$, the diffusive mode is seen as a narrow peak with the width $\Gamma$, the peak position of which is located at $\omega=0$ (so called the central peak). The width $\Gamma$ represents the decay rate of the fluctuation, that is, the inverse relaxation time. As the critical point is approached, $\Gamma$ goes to zero, implementing the critical slowing down.

As for the propagating mode, the spectral function has the form that the pole with respect to complex $\omega$ has the real part $\Omega$ as well as the imaginary part $\Gamma$. The peak for the propagating mode thus stands at $\omega=\Omega$ with the width $\Gamma$ in the spectrum. The $\Omega$ represents the frequency of the collective oscillation. For the sound wave, for example, $\Omega=v_{\mathrm{s}} q$ where $v_{\mathrm{s}}$ is the sound velocity. We note that because of the symmetry of the spectral function requested by general arguments, a propagating mode with the frequency $\Omega$ is always accompanied by a propagating mode with the frequency $-\Omega$, which propagates in the opposite direction in space. The emergence of a pair of propagating modes means that two slow variables must be involved. For the "particle mode," these two modes standing at $\omega= \pm \Omega$ in the spectrum of course correspond to the particle and anti-particle modes. As we approach the critical point, the width $\Gamma$ gets small as a consequence of the critical slowing down. At the same time, the frequency $\Omega$ also goes to zero. This phenomenon is called the softening of a propagating mode. For the sound wave, the softening implies the decrease of the sound velocity. Noting that the sound velocity measures the stiffness of the system explains the meaning of 'softening.'

It may be helpful to express the above statements in terms of the pole position in the complex $\omega$ plane. The diffusive mode is a mode that is located on the imaginary axis. Near the critical point, the pole moves on the imaginary axis toward the origin. The propagating mode is given by a pole located at an arbitrary point in the plane except on the imaginary axis, which shifts toward the origin as the critical point is approached.

Thus, both the width $\Gamma$ of the slow (either diffusive or propagating) mode and the frequency $\Omega$ of the propagating mode become small near the critical point, representing the critical slowing down and the softening, respectively. 
Concerning how $\Gamma$ and $\Omega$ go to zero, Hohenberg and Halperin have proposed the dynamic scaling hypothesis [19]: Let $\Gamma_{q}$ be a typical inverse time scale for each slow mode. We can take the width for $\Gamma_{q}$ for the diffusive mode. For the propagating mode, the frequency or width plays the role of $\Gamma_{q}$. The dynamic scaling hypothesis states that $\Gamma_{q}$ scales as a power law of the correlation length $\xi$,

$$
\Gamma_{q}(\xi)=\xi^{-z} f(q \xi)
$$

where $f$ is some function. The index $z$ is called the dynamic critical exponent and characterizes how the motion of the critical fluctuation gets slow or how the propagating mode is softened. The exponent $z$ may differ from mode to mode at one critical point. The original dynamic scaling hypothesis insists that the exponents should be identical for all the modes in the system. This seems to be a reasonable assumption because the couplings among the modes are so strong that all the modes are in the same motion. However it is known that, in many systems a single value of $z$ is not always shared among all the slow modes.

As is already described, the non-equilibrium processes near a critical point are dominated by the long wavelength fluctuations of the slow variables. It is reasonable to expect that the idea of universality, which means that the dynamics is irrelevant to the microscopic interactions in the system, is still meaningful for the dynamic case just as for the static case. As is well known, the static universality class is determined solely by the symmetry of the system. The dynamic universality class, on the other, is dependent on what kinds of slow variables are contained, besides on the symmetry. Hohenberg and Halperin have defined their dynamic universality class [14]. The rules for classification are; (i) whether or not the order parameter is conserved, (ii) what kinds of conserved quantities are possessed by the system. According to the rules, the ferromagnet and the antiferromagnet, for instance, belong to distinctive dynamic universality classes because the slow variables in the two systems are quite different, although their static universality classes are the same.

\subsection{Dynamic universality class of the chiral phase transition}

Now let us consider the chiral phase transition which is of our major interest. The static universality class of the two flavor chiral phase transition is, as is well known, the same as that of the $\mathrm{O}(4)$ Heisenberg ferro- and antiferromagnet $[1,7]$. In Ref. [7], Rajagopal and Wilczek have discussed the dynamic universality class of the chiral phase transition. They argued that the chiral phase transition belongs to the same dynamic universality class as the Heisenberg antiferromagnet, by comparing the slow variables of the both systems. The slow variables of the Heisenberg antiferromagnet are composed of the staggered magnetization $N$ which is the order parameter and not conserved, 
the magnetization $M$ which is the generator of rotation, and the energy $E$. In the chiral phase transition, on the other hand, we have the four component meson field $\phi_{\alpha}$ which plays the role of the non-conserved order parameter, the six generators of the chiral rotation $Q_{\alpha}$, the energy $E$ and the momentum $P^{i}$. We note that the momentum is lacking in the Heisenberg antiferromagnet in which the spin is sticked on the lattice sites. The difference, however, is not so crucial in the following discussions. We have only to consider the itinerant antiferromagnet rather than the Heisenberg antiferromagnet for complete correspondence. Thus, according to Hohenberg and Halperin, the two systems belong to the common dynamic universality class and we could expect the same dynamic critical behavior in both systems. Since the antiferromagnet had already been well studied [20], the analysis went on in the same way. Rajagopal and Wilczek employed the kinetic equation provided by the time dependent Ginzburg Landau theory. By applying the renormalization group method to the kinetic equation, the critical exponent $z=d / 2=3 / 2$ was obtained, where $d$ is the spatial dimensionality.

It is at this point where we should find the crucial difference between the antiferromagnet and the chiral phase transition by thinking of the slow modes rather than the slow variables in each system. Consider the disordered phase of the two systems. For the antiferromagnet, the slow mode associated with the order parameter is known to be diffusive. On the other, the slow mode for the chiral order parameter is apparently the meson mode or a "particle mode" $[6,8,10]$, which is a propagating mode. This difference should be taken seriously.

As is already mentioned, in general, we need two slow variables in order to describe a propagating mode. This is a reflection of the fact that a propagating mode always accompanies its counter partner in the spectral function. The counter partner for a "particle mode" is just an anti-particle mode. What are the two slow variables for the description of the meson mode? The answer is that the two slow variables for the meson mode are the meson field $\phi_{\alpha}$, which is the order parameter, and the canonical momentum $\pi_{\alpha}$ conjugate to $\phi_{\alpha}$. This statement is just what we would like to propose and emphasize in this paper. Note that the canonical momentum is neither an order parameter nor a conserved quantity. Since the slow modes in the antiferromagnet and in the chiral phase transition are different, and accordingly, since the slow variables in the two systems are different, it is not possible any longer to identify the dynamic universality class of the chiral phase transition with that of the antiferromagnet. In fact, as we will calculate later, the dynamic critical exponent for the chiral phase transition will be found to be $z=1-\eta / 2 \cong$ 0.98 within our framework, which is apparently not the same as $z=d / 2=$ 1.5 of the antiferromagnet. The difference of the dynamic critical exponents explicitly shows the different dynamic behavior in the two systems. 
We have found that the dynamic universality class of the chiral phase transition is not identical with that of the antiferromagnet. This means that the classification method of the dynamic universality class due to Hohenberg and Halperin is not applicable at least to the chiral phase transition. What is the problem? The classification method of Hohenberg and Halperin is essentially to compare the slow variables in the system. The usual prescription to determine the slow variable, which has been accepted without any doubt, is to gather the order parameter and the conserved quantities of the system. As we have noted, however, the canonical momentum $\pi_{\alpha}$ which is necessary for a proper description of the meson mode is neither the order parameter nor a conserved quantity. The chiral phase transiton presents us with the situation in which the order parameter and the conserved quantities do not exhaust all the slow variables necessary for a description of the slow modes. The prescription of just gleaning the order parameter and the conserved quantities for determination of the slow variables has thus broken down. Accordingly the classification method of Hohenberg and Halperin turns out insufficient because it involves just comparing the order parameter and the conserved quantities. If we consider all the slow modes or the slow variables necessary for their description, it can happen that the dynamic universality class given by Hohenberg and Halperin's method splits into finer classes.

Now what we have to do is to reanalyze the chiral phase transition, the dynamic universality class of which is not the same as that of the antiferromagnet nor that of any other critical point that has ever been considered. For that purpose, we will employ the mode coupling theory. In the analysis, we will find that a propagating mode for the meson mode appears appropriately from the meson field and the canonical momentum conjugate to it. In the next section, we will briefly review the mode coupling theory.

\section{$3 \quad$ Mode coupling theory}

The mode coupling theory is a phenomenological theory which provides us with the kinetic equation for the critical fluctuations. It was developed and established by Kawasaki $[15,16,17,18]$, and played an important role in the extensive studies of the dynamic critical phenomena in 1960-70's. Although the theory does not go beyond phenomenology, it gives us the useful quantitative predictions.

In Subsec. 3.1, we will explain the general form of the kinetic equation in the mode coupling theory. In Subsec. 3.2, as a demonstration, we will give the kinetic equation for the Heisenberg antiferromagnet in the disordered phase. 


\subsection{Kinetic equation in the mode coupling theory}

Our kinetic equation is based on the Mori equation [21]. In general, it is necessary to separate the macroscopic degrees of freedom (slow motions) from the microscopic ones (rapid motions) in order to obtain a kinetic equation. This can be implemented by the projection operator method [22]. The Mori equation is derived by applying the projection operator method to the microscopic equation of motion, i.e., the Heisenberg equation. The Mori equation deals with only the slow motions directly. In a sense, the Mori equation is just the Heisenberg equation after the rapid fluctuations are integrated out. Thus we can state that the kinetic equation in the mode coupling theory originates from the microscopic Heisenberg equation.

The explicit form of the kinetic equation in the mode coupling theory is given by

$$
\begin{aligned}
\frac{\mathrm{d}}{\mathrm{d} t} a_{\vec{q}}^{j}(t)= & \sum_{l}\left(\mathrm{i} \omega_{\vec{q}}^{j l}-\frac{k_{\mathrm{B}}}{\chi_{\vec{q}}^{l}} L_{j l}^{0}\right) a_{\vec{q}}^{l}(t) \\
& +\frac{\mathrm{i}}{2} \sum_{\vec{k}} \sum_{l m} \Omega_{j ; l m}\left(\chi_{\vec{k}}^{l} \chi_{\vec{q}-\vec{k}}^{m}\right)^{-\frac{1}{2}}\left(a_{\vec{k}}^{l} a_{\vec{q}-\vec{k}}^{m}-\left\langle a_{\vec{k}}^{l} a_{\vec{q}-\vec{k}}^{m}\right\rangle_{\mathrm{eq}}\right)+f_{\vec{q}}^{j},
\end{aligned}
$$

where

$$
\begin{aligned}
& \omega_{\vec{q}}^{j l}=-k_{\mathrm{B}} T\left(\chi_{\vec{q}}^{l}\right)^{-1}\left\langle\left[a_{\vec{q}}^{j}, a_{\vec{q}}^{l \dagger}\right]\right\rangle_{\mathrm{eq}}, \\
& \Omega_{j ; l m}=-k_{\mathrm{B}} T\left(\chi_{\vec{k}}^{l} \chi_{\vec{q}-\vec{k}}^{m}\right)^{-\frac{1}{2}}\left\{\left\langle\left[a_{\vec{q}}^{j}, a_{\vec{k}}^{l \dagger} a_{\vec{q}-k}^{m \dagger}\right]\right\rangle_{\mathrm{eq}}\right. \\
&\left.\quad-\sum_{p}\left\langle\left[a_{\vec{q}}^{j}, a_{\vec{q}}^{p \dagger}\right]\right\rangle_{\mathrm{eq}} \chi_{\vec{q}}^{p-1}\left(a_{\vec{q}}^{p}, a_{\vec{k}}^{l \dagger} a_{\vec{q}-\vec{k}}^{m \dagger}\right)\right\} .
\end{aligned}
$$

The $a_{\vec{q}}^{j}$ is the microscopic expression for the Fourier transformed fluctuation of the $j$ th slow variable. The $\chi_{\vec{q}}^{j}$ is the static susceptibility given by

$$
\chi_{\vec{q}}^{j} \delta_{j l}=\left(a_{\vec{q}}^{j}, a_{\vec{q}}^{l}\right) \cong\left\langle a_{\vec{q}}^{j} a_{\vec{q}}^{l}\right\rangle_{\mathrm{eq}},
$$

where the inner product $(F, G)$ is defined by

$$
(F, G) \equiv \frac{1}{\beta} \int_{0}^{\beta} \mathrm{d} \lambda\left\langle e^{\lambda H} F e^{-\lambda H} G\right\rangle_{\text {eq }}
$$

with the inverse temperature $\beta=1 / k_{\mathrm{B}} T$ and the Hamiltonian $H$. In the above expressions, $\langle\cdots\rangle_{\text {eq }}$ denotes the statistical average in the equilibrium ensemble. In the following, we will omit the index 'eq' except for emphasizing it. 
There are three terms in the right-hand side of Eq. (3.1), which are the linear term, the nonlinear term and the noise term $f_{\vec{q}}^{j}$. The equation without the nonlinear term describes the free motion of the slow modes. The nonlinear term corresponds to the interactions among them.

The linear term consists of the frequency term and the dissipative term, which give the peak position and its width in the spectral function of the slow variables, respectively. The frequency matrix $\omega_{j l}$ is given by the statistical average of the commutator of the slow variables in the equilibrium ensemble, as is shown in Eq. (3.2). For the classical system, the commutator is replaced by the Poisson brackets. By diagonalizing the frequency matrix, we obtain the normal coordinates out of the slow variables for the individual slow modes. The nonzero eigenvalue means the appearance of the propagating mode for the associated slow variables, while the zero eigenvalue corresponds to the diffusive mode.

The $L^{0}$ in the dissipative term gives the Onsager (transport) coefficient. Within the present framework, $L^{0}$ is given by the correlation among only the short wavelength fluctuations, and thus does not show any anomalous behavior in itself. In general, however, the Onsager coefficient can be divergent at a critical point because of the effect of the long wavelength fluctuation. In the mode coupling theory, actually, the renormalization coming from the nonlinear term can give rise to the divergence in the Onsager coefficient. In order to emphasize that $L^{0}$ is a bare or unrenormalized quantity, the suffix 0 is put on $L$.

The nonlinear term, which represents the interactions among the slow modes, is called the mode coupling term. It is known that the couplings among the fluctuations of the slow variables become very strong near a critical point [23]. Thus the mode coupling term is crucial for the critical dynamics. One of the effects of the mode coupling term is the possible divergence of the Onsager coefficient mentioned above. If the mode coupling term is dropped, the theory reduces to the van Hove theory [24].

We note that the coefficients in the kinetic equation are all given by the statistical average in the equilibrium ensemble and determined by the static properties of the system. The dynamic nature is contained in the kinetic equation itself. Thus in the mode coupling theory, we input the static information into the kinetic equation in order to obtain the dynamic information. In particular, the criticality is furnished by the singularity of the static susceptibilities. The anomalous behavior at a critical point such as the critical slowing down and the softening is induced by the divergence of the static susceptibilities. One consequence is that the dynamic critical exponents are expressed in term of the static critical exponents in this description, as we will see later. 


\subsection{Kinetic equation for the Heisenberg antiferromagnet}

For illustration of the general formalism in the previous section, we here give the kinetic equation for the Heisenberg antiferromagnet in the disordered phase $[15,16,17]$, which would be useful for the comparison with the chiral phase transition later.

The Hamiltonian of the Heisenberg antiferromagnet is given by

$$
H=-\sum_{i \neq j} J_{i j} \vec{s}_{i} \cdot \vec{s}_{j}
$$

where $\vec{s}_{i}$ is the spin operator vector put on the $i$ th lattice site which satisfies

$$
\left[s_{i}^{\alpha}, s_{j}^{\beta}\right]=\mathrm{i} \epsilon^{\alpha \beta \gamma} \delta_{i j} s_{i}^{\gamma} \quad(\alpha, \beta, \gamma=x, y, z) .
$$

The $J_{i j}$ represents the exchange interaction and has a negative sign for the antiferromagnet.

What we should do in the first place is to determine the slow variables. The slow variables are made up of the staggered magnetization (order parameter), the magnetization and the energy (conserved quantities).

The Fourier transforms of the staggered magnetization $\sigma_{\vec{k}}^{\alpha}$, the magnetization $\mu_{\vec{k}}^{\alpha}$ and the energy density $e_{\vec{k}}$ are given by

$$
\begin{aligned}
\sigma_{\vec{k}}^{\alpha} & =\sum_{i} \eta^{i} e^{-\mathrm{i} \vec{k} \cdot \vec{r}_{i}}\left(s_{i}^{\alpha}-\left\langle s_{i}^{\alpha}\right\rangle\right), \\
\mu_{\vec{k}}^{\alpha} & =\sum_{i} e^{-\mathrm{i} \vec{k} \cdot \vec{r}_{i}}\left(s_{i}^{\alpha}-\left\langle s_{i}^{\alpha}\right\rangle\right), \\
e_{\vec{k}} & =\sum_{i} e^{-\mathrm{i} \vec{k} \cdot \vec{r}_{i}}(-) \sum_{j} J_{i j}\left(\vec{s}_{i} \cdot \vec{s}_{j}-\left\langle\vec{s}_{i} \cdot \vec{s}_{j}\right\rangle\right) .
\end{aligned}
$$

where $\eta^{i}$ has an opposite sign for the nearest neighbors.

The frequency matrix $\omega_{\vec{q}}$ can be calculated by the commutation relation between the spin variables. We find that the matrix elements are all zero. This indicates that all the slow modes are diffusive. Especially note that the slow mode for the staggered magnetization is diffusive as it should be.

The dissipation terms can be found from the macroscopic hydrodynamics. Since the magnetization and the energy are conserved quantities, we have

$$
\begin{aligned}
k_{\mathrm{B}} L_{\mu \vec{k}, \mu \vec{k}}^{0} / \chi_{\mu \vec{k}} & =k^{2} L_{\mu}^{0} / \chi_{\mu \vec{k}}, \\
k_{\mathrm{B}} L_{e \vec{k}, e \vec{k}}^{0} / \chi_{e \vec{k}} & =k^{2} \lambda^{0} / C_{\vec{k}},
\end{aligned}
$$


which define the 'bare' Onsager coefficients. In particular, $\lambda^{0}$ is the 'bare' thermal conductivity. The $C_{\vec{k}}$ is the $\vec{k}$-dependent heat capacity per spin. For the staggered magnetization which is not a conserved quantity, we have

$$
k_{\mathrm{B}} L_{\sigma \vec{k}, \sigma \vec{k}}^{0} / \chi_{\sigma \vec{k}}=L_{\sigma}^{0} / \chi_{\sigma \vec{k}}
$$

The susceptibilities $\chi$ 's are defined by

$$
\begin{aligned}
& \chi_{\sigma \vec{k}}=\frac{1}{N}\left(\sigma_{\vec{k}}^{\alpha}, \sigma_{-\vec{k}}^{\alpha}\right), \\
& \chi_{\mu \vec{k}}=\frac{1}{N}\left(\mu_{\vec{k}}^{\alpha}, \mu_{-\vec{k}}^{\alpha}\right), \\
& \chi_{e \vec{k}}=\frac{1}{N}\left(e_{\vec{k}}, e_{-\vec{k}}\right),
\end{aligned}
$$

where $N$ is the total number of the spin site in the system. The coefficients of the nonlinear term, $\Omega_{j ; l m}$, can be computed with the spin commutation relation.

Finally we can obtain the kinetic equation,

$$
\begin{aligned}
& \frac{\mathrm{d}}{\mathrm{d} t} \vec{\sigma}_{\vec{q}}=-\frac{L_{\sigma}^{0}}{\chi_{\sigma \vec{q}}} \vec{\sigma}_{\vec{q}}+\frac{k_{\mathrm{B}} T}{N} \sum_{\vec{k}}\left(\frac{1}{\chi_{\sigma \vec{k}}}-\frac{1}{\chi_{\mu \vec{q}-\vec{k}}}\right) \vec{\sigma}_{\vec{k}} \times \vec{\mu}_{\vec{q}-\vec{k}}+f_{\vec{q}}^{\sigma}, \\
& \frac{\mathrm{d}}{\mathrm{d} t} \vec{\mu}_{\vec{q}}=-q^{2} \frac{L_{\mu}^{0}}{\chi_{\mu \vec{q}}} \vec{\mu}_{\vec{q}}+\frac{k_{\mathrm{B}} T}{2 N} \sum_{\vec{k}}\left(\frac{1}{\chi_{\mu \vec{k}}}-\frac{1}{\chi_{\mu \vec{q}-\vec{k}}}\right) \vec{\mu}_{\vec{k}} \times \vec{\mu}_{\vec{q}-\vec{k}} \\
& +\frac{k_{\mathrm{B}} T}{2 N} \sum_{\vec{k}}\left(\frac{1}{\chi_{\sigma \vec{k}}}-\frac{1}{\chi_{\sigma \vec{q}-\vec{k}}}\right) \vec{\sigma}_{\vec{k}} \times \vec{\sigma}_{\vec{q}-\vec{k}}+f_{\vec{q}}^{\mu}, \\
& \frac{\mathrm{d}}{\mathrm{d} t} e_{\vec{q}}=-q^{2} \frac{\lambda^{0}}{C_{\vec{q}}} e_{\vec{q}}+f_{\vec{q}}^{e} .
\end{aligned}
$$

Note that the energy mode decouples from the magnetization and staggered magnetization modes.

\section{Slow modes near the chiral phase transition in the $\mathrm{O}(2)$ sigma model}

In this section, we apply the mode coupling theory to the linear sigma model for investigation of the chiral phase transition. We examine what kind of slow modes, diffusive or propagating modes, appears near the critical point. For that purpose, it is sufficient to consider the frequency matrix $\omega$ in the kinetic equation. If the matrix element gives a finite value, it means that there appears a propagating mode in the associated slow variable. The zero matrix element indicates a diffusive mode. For simplicity, we consider the $\mathrm{O}(2)$ linear sigma 
model. The Lagrangian is

$$
\mathcal{L}=\frac{1}{2}\left[\left(\partial_{\mu} \phi_{1}\right)^{2}+\left(\partial_{\mu} \phi_{2}\right)^{2}\right]-\frac{1}{2} \mu^{2}\left({\phi_{1}}^{2}+{\phi_{2}}^{2}\right)-\frac{\lambda}{4}\left(\phi_{1}{ }^{2}+{\phi_{2}}^{2}\right)^{2},
$$

where $\phi_{a}$ with $a=1,2$ is the meson field, and $\mu$ and $\lambda$ are the bare mass and coupling constant respectively. For definiteness, we assume that the $\phi_{1}$ takes a finite vacuum expectation value $\left\langle\phi_{1}\right\rangle=\phi$ in the ordered phase. Thus the fluctuations of $\phi_{1}$ and $\phi_{2}$ correspond to the $\sigma$ and $\pi$ meson modes, respectively.

As we argued in Subsec. 2.2, the slow variables for the chiral phase transition are composed of the following; the meson field $\phi(\vec{x}, t)$ (order parameter), the canonical momentum $\pi(\vec{x}, t)$, the chiral charge density $Q(\vec{x}, t)$, the energy density $E(\vec{x}, t)$ and the momentum density $P^{i}(\vec{x}, t)$. The last three of these slow variables are the conserved quantities. Note that there is only one chiral charge, which is contrasted with the six charges in the $\mathrm{O}(4)$ case. The microscopic expressions for $Q(\vec{x}, t), E(\vec{x}, t), P^{i}(\vec{x}, t)$ are given by

$$
\begin{aligned}
Q(\vec{x}, t) & =\left(\pi_{1} \phi_{2}-\pi_{2} \phi_{1}\right)(\vec{x}, t), \\
E(\vec{x}, t) & =\left[\frac{1}{2} \pi_{a}{ }^{2}+\frac{1}{2}\left(\vec{\nabla} \phi_{a}\right)^{2}+\frac{1}{2} \mu^{2} \phi_{a}{ }^{2}+\frac{\lambda}{4}\left(\phi_{a}{ }^{2}\right)^{2}\right](\vec{x}, t), \\
P^{i}(\vec{x}, t) & =-\left(\pi_{a} \nabla^{i} \phi_{a}\right)(\vec{x}, t) .
\end{aligned}
$$

Here, as usual, we expand $\phi_{a}(\vec{x}, t)$ and $\pi_{a}(\vec{x}, t)$ into the normal modes in terms of the creation and annihilation operators.

$$
\begin{aligned}
& \phi_{a}(\vec{x}, t)=\frac{1}{\sqrt{V}} \sum_{\vec{k}} \frac{1}{\sqrt{2 \omega_{a \vec{k}}}}\left(a_{a \vec{k}}(t)+a_{a-\vec{k}}^{\dagger}(t)\right) e^{\mathrm{i} \vec{k} \cdot \vec{x}}, \\
& \pi_{a}(\vec{x}, t)=\frac{1}{\sqrt{V}} \sum_{\vec{k}}(-\mathrm{i}) \sqrt{\frac{\omega_{a \vec{k}}}{2}}\left(a_{a \vec{k}}(t)-a_{a-\vec{k}}^{\dagger}(t)\right) e^{\mathrm{i} \vec{k} \cdot \vec{x}},
\end{aligned}
$$

where $V$ is the volume of the system. The dispersion relation $\omega_{a \vec{k}}$ is generally complicated as a consequence of the interaction. For the free system $(\lambda=0)$, the Klein-Gordon equation gives $\omega_{\vec{k}}=\sqrt{\vec{k}^{2}+\mu^{2}}$. We will determine $\omega_{a \vec{k}}$ at the final stage of consideration by imposing a consistency condition.

The usual quantization conditions

$$
\begin{aligned}
& {\left[\phi_{a}(\vec{x}, t)-\left\langle\phi_{a}\right\rangle, \pi_{b}(\vec{y}, t)\right]=\mathrm{i} \delta_{a b} \delta(\vec{x}-\vec{y}),} \\
& {\left[\phi_{a}(\vec{x}, t)-\left\langle\phi_{a}\right\rangle, \phi_{b}(\vec{y}, t)-\left\langle\phi_{b}\right\rangle\right]=\left[\pi_{a}(\vec{x}, t), \pi_{b}(\vec{y}, t)\right]=0,}
\end{aligned}
$$


or

$$
\begin{aligned}
& {\left[a_{a \vec{k}}(t), a_{b \vec{k}^{\prime}}^{\dagger}(t)\right]=\delta_{a b} \delta_{\vec{k} \vec{k}^{\prime}},} \\
& {\left[a_{a \vec{k}}(t), a_{b \vec{k}^{\prime}}(t)\right]=\left[a_{a \vec{k}}^{\dagger}(t), a_{b \vec{k}^{\prime}}^{\dagger}(t)\right]=0,}
\end{aligned}
$$

provide us with the algebra to calculate the frequency matrix.

The Fourier components of the fluctuation of the slow variables are given by

$$
\begin{aligned}
\phi_{a \vec{q}}(t) & =\frac{1}{\sqrt{V}} \int \mathrm{d}^{3} x e^{-\mathrm{i} \vec{q} \cdot \vec{x}}\left(\phi_{a}(\vec{x}, t)-\left\langle\phi_{a}\right\rangle_{\mathrm{eq}}\right), \\
\pi_{a \vec{q}}(t) & =\frac{1}{\sqrt{V}} \int \mathrm{d}^{3} x e^{-\mathrm{i} \vec{q} \cdot \vec{x}} \pi_{a}(\vec{x}, t), \\
Q_{\vec{q}}(t) & =\epsilon_{a b} \frac{1}{\sqrt{V}} \int \mathrm{d}^{3} x e^{-\mathrm{i} \vec{q} \cdot \vec{x}} \pi_{a} \phi_{b}(\vec{x}, t), \\
E_{\vec{q}}(t)= & \frac{1}{\sqrt{V}} \int \mathrm{d}^{3} x e^{-\mathrm{i} \vec{q} \cdot \vec{x}}\left[\left(\frac{1}{2} \pi_{a}{ }^{2}+\frac{1}{2}\left(\vec{\nabla} \phi_{a}\right)^{2}+\frac{1}{2} \mu^{2} \phi_{a}{ }^{2}\right.\right. \\
& \left.\left.+\frac{\lambda}{4}\left(\phi_{a}^{2}\right)^{2}\right)(\vec{x}, t)-\langle\text { the same expression }\rangle_{\mathrm{eq}}\right], \\
P_{\vec{q}}^{i}(t)= & \frac{1}{\sqrt{V}} \int \mathrm{d}^{3} x e^{-\mathrm{i} \vec{q} \cdot \vec{x}}\left(-\pi_{a} \nabla^{i} \phi_{a}\right)(\vec{x}, t),
\end{aligned}
$$

where $\epsilon_{a b}$ is the total antisymmetric tensor with $\epsilon_{12}=1$. Equivalently we have

$$
\begin{aligned}
& \phi_{a \vec{q}}(t)=\frac{1}{\sqrt{2 \omega_{a \vec{k}}}}\left(\left(a_{a \vec{q}}+a_{a-\vec{q}}^{\dagger}\right)-\left\langle a_{a \vec{q}}+a_{a-\vec{q}}^{\dagger}\right\rangle_{\mathrm{eq}}\right), \\
& \pi_{a \vec{q}}(t)=(-\mathrm{i}) \sqrt{\frac{\omega_{a \vec{q}}}{2}}\left(a_{a \vec{q}}(t)-a_{a-\vec{q}}^{\dagger}(t)\right), \\
& Q_{\vec{q}}(t)=\epsilon_{a b} \frac{1}{\sqrt{V}} \sum_{\vec{k}} \frac{-\mathrm{i}}{2} \sqrt{\frac{\omega_{a \vec{k}}}{\omega_{b \vec{q}-\vec{k}}}}\left(a_{a \vec{k}}-a_{a-\vec{k}}^{\dagger}\right)\left(a_{b \vec{q}-\vec{k}}+a_{b \vec{k}-\vec{q}}^{\dagger}\right), \\
& E_{\vec{q}}(t)=\frac{1}{2} \frac{1}{\sqrt{V}} \sum_{\vec{k}} \frac{-1}{2} \sqrt{\omega_{a \vec{k}} \omega_{a \vec{q}-\vec{k}}}\left(a_{a \vec{k}}-a_{a-\vec{k}}^{\dagger}\right)\left(a_{a \vec{q}-\vec{k}}-a_{a \vec{k}-\vec{q}}^{\dagger}\right) \\
& +\frac{1}{2} \frac{1}{\sqrt{V}} \sum_{\vec{k}}\left[-\vec{k} \cdot(\vec{q}-\vec{k})+\mu^{2}\right] \frac{1}{2} \frac{1}{\sqrt{\omega_{a \vec{k}} \omega_{a \vec{q}-\vec{k}}}}\left(a_{a \vec{k}}+a_{a-\vec{k}}^{\dagger}\right)\left(a_{a \vec{q}-\vec{k}}+a_{a \vec{k}-\vec{q}}^{\dagger}\right) \\
& +\frac{\lambda}{4} \frac{1}{V^{\frac{3}{2}}} \sum_{\overrightarrow{k_{1}} \cdots \overrightarrow{k_{4}}} \frac{1}{4} \delta_{\vec{q}, \overrightarrow{k_{1}}+\cdots+\overrightarrow{k_{4}}} \frac{1}{\sqrt{\omega_{a \overrightarrow{k_{1}}} \omega_{a \overrightarrow{k_{2}}} \omega_{b \overrightarrow{k_{3}}} \omega_{b \overrightarrow{k_{4}}}}} \\
& \times\left(a_{a \overrightarrow{k_{1}}}+a_{a-\overrightarrow{k_{1}}}^{\dagger}\right)\left(a_{a \overrightarrow{k_{2}}}+a_{a-\overrightarrow{k_{2}}}^{\dagger}\right)\left(a_{b \overrightarrow{k_{3}}}+a_{b-\overrightarrow{k_{3}}}^{\dagger}\right)\left(a_{b \overrightarrow{k_{4}}}+a_{b-\overrightarrow{k_{4}}}^{\dagger}\right) \\
& -\langle\text { the same expression }\rangle_{\text {eq }} \delta_{\vec{q}, 0} \text {, } \\
& P_{\vec{q}}^{i}(t)=-\frac{1}{\sqrt{V}} \sum_{\vec{k}} \frac{1}{2}(q-k)^{i} \sqrt{\frac{\omega_{a \vec{k}}}{\omega_{b \vec{q}-\vec{k}}}}\left(a_{a \vec{k}}-a_{a-\vec{k}}^{\dagger}\right)\left(a_{a \vec{q}-\vec{k}}+a_{a \vec{k}-\vec{q}}^{\dagger}\right) \text {. }
\end{aligned}
$$

The frequency matrix element is given by the commutator of each slow variable 
in the mode coupling theory. By noting that the commutator between $\phi_{a}$ and $\pi_{a}$ is not zero, we can see a propagating mode appearing from these two slow variables. This propagating mode should and can be identified with the meson mode, which will be discussed later. Note that if we do not include the canonical momentum into the group of the slow variables just following the usual prescription for determination of the slow variable, this propagating mode would not appear and the order parameter fluctuation would undesirably show diffusive behavior. The canonical momentum plays an essential role in the chiral phase transition.

The inclusion of $\pi_{a}$ is found to be reasonable if we consider the microscopic equation of motion, that is, the Heisenberg equation. The Heisenberg equation for the meson field consists of the two equations for the variables of $\phi$ and $\pi$ which are conjugate to each other;

$$
\begin{aligned}
\mathrm{i} \frac{\mathrm{d}}{\mathrm{d} t} \phi(\vec{x}, t) & =[\phi(\vec{x}, t), H], \\
\mathrm{i} \frac{\mathrm{d}}{\mathrm{d} t} \pi(\vec{x}, t) & =[\pi(\vec{x}, t), H],
\end{aligned}
$$

where $H$ is the Hamiltonian of the system. From these two equations, we obtain the Klein-Gordon equation for the meson dynamics. ${ }^{2}$ Since our kinetic equation in the mode coupling theory is based on the Mori equation which is derived from the Heisenberg equation, it is natural that we need $\pi_{a}$ as the degree of freedom for a description of the meson dynamics.

Turning to the case of the antiferromagnet, we realize that the Heisenberg equation for the spin variables is

$$
\mathrm{i} \frac{\mathrm{d} s_{i}^{\alpha}}{\mathrm{d} t}=\left[s_{i}^{\alpha}, H\right] \quad(\alpha=x, y, z)
$$

with $H$ being given by Eq. (3.6). Thus the staggered magnetization $\vec{\sigma}=$ $\left(\sigma^{x}, \sigma^{y}, \sigma^{z}\right)$ corresponding to the spin variable $\vec{s}=\left(s^{x}, s^{y}, s^{z}\right)$ should be the degree of freedom for the dynamics of the antiferromagnet. In fact, from the variable of the staggered magnetization arises a diffusive mode as it should be, as we have already seen in Sec. 3 .

The canonical momentum as a slow variable is itself nothing new in the chiral phase transition. We know at least two examples of critical points in which the canonical momentum enters into the member of the slow variable.

2 We note that the Klein-Gordon equation is of second order with respect to the time derivative while the Heisenberg equation is of first order. In order to reproduce the Klein-Gordon equation from the Heisenberg equation, it is inevitable to set up the simultaneous equations for two variables. 
One is the $\lambda$ transition in the superfluid. The order parameter of the transition is given by the wavefunction of the Bose condensate $\Phi$. Because the wavefunction is a complex variable, the order parameter consists of the two components, i.e., $\Phi$ and $\Phi^{\dagger}$. The wavefunction $\Phi$ obeys the non-relativistic Schödinger equation, and as is well known, the canonical momentum conjugate to $\Phi$ is given by $\Phi^{\dagger}$. From the variables of $\Phi$ and $\Phi^{\dagger}$ (and the entropy density), there appears the second sound mode which is a propagating mode (and one diffusive mode) in the superfluid transition.

The other example is associated with the spin wave mode in the ferromagnet. In the ordered phase, supposing that the spontaneous magnetization points in the $z$ direction, the transverse fluctuations of the magnetization, $M_{x}$ and $M_{y}$, make up the spin wave. We can regard the two variables of $M_{ \pm}=M_{x} \pm \mathrm{i} M_{y}$ as conjugate to each other.

Note that in the above two examples, the conjugate variable can be included into the member of the slow variable just by collecting the order parameter and the conserved quantity. In the chiral phase transition, however, the canonical momentum cannot be the slow variable by the prescription. This is nothing but the new feature of the chiral phase transition that we have not encountered in the systems so far considered in Ref. [14].

Alternatively to $\phi_{a}$ and $\pi_{a}$, we can take

$$
\frac{1}{\sqrt{2 \omega_{a \vec{q}}}}\left(a_{a \vec{q}}-\left\langle a_{a \vec{q}}\right\rangle\right) \quad \text { and } \quad \frac{1}{\sqrt{2 \omega_{a \vec{q}}}}\left(a_{a-\vec{q}}^{\dagger}-\left\langle a_{a-\vec{q}}^{\dagger}\right\rangle\right)
$$

for the two variables. These are obtained by dividing the meson field $\phi_{a \vec{q}}$, and are just a liner transformation of $\phi_{a}$ and $\pi_{a}$. In the following discussions, we will employ $a_{a}$ and $a_{a}^{\dagger}$ instead of $\phi_{a}$ and $\pi_{a}$ for the two degrees of freedom. This is because $a_{a}$ and $a_{a}^{\dagger}$ give rise to the diagonal frequency matrix as we will see later and are more convenient.

Thus for the two degrees of freedom to describe the meson mode, we will adopt

$$
\begin{aligned}
\phi_{a \vec{q}}(t) & \equiv \frac{1}{\sqrt{2 \omega_{a \vec{q}}}}\left(a_{a \vec{q}}-\left\langle a_{a \vec{q}}\right\rangle\right), \\
\phi_{a-\vec{q}}^{\dagger}(t) & \equiv \frac{1}{\sqrt{2 \omega_{a \vec{q}}}}\left(a_{a-\vec{q}}^{\dagger}-\left\langle a_{a-\vec{q}}^{\dagger}\right\rangle\right) .
\end{aligned}
$$

Now we calculate the frequency matrix $\omega_{j l}$ given by

$$
\omega_{j l}=-k_{\mathrm{B}} T\left(\chi_{l}\right)^{-1}\left\langle\left[A_{j}, A_{l}^{\dagger}\right]\right\rangle
$$

for the slow variables of

$$
A_{j}=\left\{\phi_{1 \vec{q}}, \phi_{1-\vec{q}}^{\dagger}, \phi_{2 \vec{q}}, \phi_{2-\vec{q}}^{\dagger}, Q_{\vec{q}}, E_{\vec{q}}, P_{\vec{q}}^{i}\right\},
$$


where $j$ on $A_{j}$ specifies each slow variable. The static susceptibilities for $\phi_{a \vec{q}}$ and $\phi_{a-\vec{q}}^{\dagger}$ are given by

$$
\begin{aligned}
\chi_{a \vec{q}} & \equiv\left(\phi_{a \vec{q}},\left(\phi_{a \vec{q}}\right)^{\dagger}\right), \\
\chi_{a^{\dagger} \vec{q}} & \equiv\left(\phi_{a-\vec{q}}^{\dagger},\left(\phi_{a-\vec{q}}^{\dagger}\right)^{\dagger}\right)=\left(\phi_{a-\vec{q}},\left(\phi_{a-\vec{q}}\right)^{\dagger}\right)=\chi_{a \vec{q}},
\end{aligned}
$$

where the last equality holds because the susceptibility is a function of $|\vec{q}|^{2}$. The other susceptibilities are defined similarly by

$$
\begin{aligned}
\chi_{Q \vec{q}} & \equiv\left(Q_{\vec{q}}, Q_{\vec{q}}^{\dagger}\right), \\
\chi_{e \vec{q}} & \equiv\left(E_{\vec{q}}, E_{\vec{q}}^{\dagger}\right), \\
\chi_{p \vec{q}} & \equiv\left(P_{\vec{q}}^{i}, P_{\vec{q}}^{i \dagger}\right),
\end{aligned}
$$

where $\chi_{p \vec{q}}$ does not depend on $i$.

Firstly, let us consider the disordered phase, in which $\phi_{1}$ and $\phi_{2}$ are degenerated. The frequency matrix is calculated to be

$$
\omega_{j l}=\left(\begin{array}{ccccccc}
-\frac{k_{\mathrm{B}} T}{2 \omega_{1 \vec{q}} \chi_{1 \vec{q}}} & 0 & 0 & 0 & 0 & 0 & 0 \\
0 & \frac{k_{\mathrm{B}} T}{2 \omega_{1 \vec{q}} \chi_{1 \vec{q}}} & 0 & 0 & 0 & 0 & 0 \\
0 & 0 & -\frac{k_{\mathrm{B}} T}{2 \omega_{2 \vec{q}} \chi_{2 \vec{q}}} & 0 & 0 & 0 & 0 \\
0 & 0 & 0 & \frac{k_{\mathrm{B}} T}{2 \omega_{2 \vec{q}} \chi_{2 \vec{q}}} & 0 & 0 & 0 \\
0 & 0 & 0 & 0 & 0 & 0 & 0 \\
0 & 0 & 0 & 0 & 0 & 0 & -\frac{D}{\chi_{p \vec{q}}} q^{i} \\
0 & 0 & 0 & 0 & 0-\frac{D}{\chi_{e \vec{q}}} q^{i} & 0
\end{array}\right),
$$

where

$$
D=k_{\mathrm{B}} T \frac{1}{V} \sum_{\vec{k}} 2\left(\omega_{a \vec{k}}^{2}+\frac{1}{3} \vec{k}^{2}\right) \chi_{a \vec{k}},
$$

and the matrix elements are placed in the order of Eq. (4.28). We note that there appear propagating modes for $\phi_{1}$ and $\phi_{2}$, which should be identified with the $\sigma$ and $\pi$ meson modes. The kinetic equation without the dissipative and nonlinear terms is

$$
\frac{\mathrm{d}}{\mathrm{d} t} \phi_{a \vec{q}}=-\mathrm{i} \frac{k_{\mathrm{B}} T}{2 \omega_{a \vec{q}} \chi_{a \vec{q}}} \phi_{a \vec{q}}, \quad \frac{\mathrm{d}}{\mathrm{d} t} \phi_{a-\vec{q}}^{\dagger}=\mathrm{i} \frac{k_{\mathrm{B}} T}{2 \omega_{a \vec{q}} \chi_{a \vec{q}}} \phi_{a-\vec{q}}^{\dagger} .
$$

Here we impose a consistency condition that the frequency coincide with the dispersion $\omega_{a \vec{q}}$, that is,

$$
\frac{k_{\mathrm{B}} T}{2 \omega_{a \vec{q}} \chi_{a \vec{q}}}=\omega_{a \vec{q}}
$$


This is justified because in the mode coupling theory, the kinetic equation without the dissipation and mode coupling term should reproduce the Heisenberg equation [16]. In fact, the equations given by Eq. (4.36) with Eq. (4.37) substituted are combined into

$$
\begin{aligned}
\frac{\mathrm{d}}{\mathrm{d} t}\left(\phi_{a \vec{q}}+\phi_{a-\vec{q}}^{\dagger}\right) & =-\mathrm{i} \omega_{a \vec{q}}\left(\phi_{a \vec{q}}-\phi_{a-\vec{q}}^{\dagger}\right), \\
\frac{\mathrm{d}}{\mathrm{d} t}(-\mathrm{i}) \omega_{a \vec{q}}\left(\phi_{a \vec{q}}-\phi_{a-\vec{q}}^{\dagger}\right) & =-\omega_{a \vec{q}}^{2}\left(\phi_{a \vec{q}}+\phi_{a-\vec{q}}^{\dagger}\right),
\end{aligned}
$$

which give the Heisenberg equation or the Klein-Gordon equation with the dispersion relation $\omega_{a \vec{q}}$. The condition (4.37) determines the dispersion relation as

$$
\omega_{a \vec{q}}=\sqrt{\frac{k_{\mathrm{B}} T}{2 \chi_{a \vec{q}}}} .
$$

In order to find $\chi_{a \vec{q}}$, consider the susceptibility for the field $\phi(\vec{x})$ itself. In the Ornstein-Zernike approximation, the susceptibility is written as

$$
\left(\phi(\vec{x}), \phi(\vec{y})^{\dagger}\right)=\frac{1}{V} \sum_{\vec{k}} \frac{k_{\mathrm{B}} T Z}{\vec{k}^{2}+m^{2}} e^{\mathrm{i} \vec{k} \cdot(\vec{x}-\vec{y})},
$$

where $m$ is the static screening mass. On the other hand,

$$
\begin{aligned}
\left(\phi(\vec{x}), \phi(\vec{y})^{\dagger}\right) & =\left(\frac{1}{\sqrt{V}} \sum_{\vec{k}}\left(\phi_{\vec{k}}+\phi_{-\vec{k}}^{\dagger}\right) e^{\mathrm{i} \vec{k} \cdot \vec{x}}, \frac{1}{\sqrt{V}} \sum_{\vec{k}^{\prime}}\left(\phi_{\vec{k}^{\prime}}^{\dagger}+\phi_{-\vec{k}^{\prime}}\right) e^{-\mathrm{i} \vec{k}^{\prime} \cdot \vec{y}}\right) \\
& =\frac{1}{V} \sum_{\vec{k}} 2 \chi_{\vec{k}} e^{i \vec{k} \cdot(\vec{x}-\vec{y})} .
\end{aligned}
$$

By comparing the two expressions, we find

$$
2 \chi_{\vec{k}}=\frac{k_{\mathrm{B}} T Z}{\vec{k}^{2}+m^{2}} .
$$

Thus we obtain the dispersion relation

$$
\omega_{a \vec{q}}=Z^{-\frac{1}{2}} \sqrt{\vec{q}^{2}+m^{2}} .
$$

We should note that the static screening mass $m$ plays the role of the pole mass in this framework. As we approach the critical point, $m$ goes to zero, which describes the softening of the meson modes. The way of the softening, or the way how $m$ gets small, can be determined by the static scaling law, i.e., $m \sim \xi^{-1}$. The renormalization constant $Z$ yields the anomalous dimension as $Z \sim \xi^{\eta}$, which leads to the dynamic critical exponent $1-\eta / 2$ as is already advertised. Although the present argument does not take account of the nonlinear or mode coupling terms which are important near the critical point, the result does not alter even when they are properly taken care of. The full analysis will be given in Sec. 6 . 
We can see that the slow mode for the chiral charge is diffusive. Moreover, the energy and the longitudinal component of the momentum couple to give rise to a propagating mode, while the transverse components give diffusive modes. The kinetic equations for the energy and the momentum are written as

$$
\begin{aligned}
\frac{\mathrm{d}}{\mathrm{d} t} E_{\vec{q}} & =-\mathrm{i} \frac{D}{\chi_{p \vec{q}}} q^{i} P_{\vec{q}}^{\mathrm{L} i}, \\
\frac{\mathrm{d}}{\mathrm{d} t} P_{\vec{q}}^{\mathrm{L} i} & =-\mathrm{i} \frac{D}{\chi_{e \vec{q}}} q^{i} E_{\vec{q}}, \\
\frac{\mathrm{d}}{\mathrm{d} t} P_{\vec{q}}^{\mathrm{T} i} & =0,
\end{aligned}
$$

where we defined $P_{\vec{q}}^{\mathrm{L} i}$ and $P_{\vec{q}}^{\mathrm{T} i}$ by

$$
P_{\vec{q}}^{i}=\left(\delta^{i j}-\frac{q^{i} q^{j}}{\vec{q}^{2}}\right) P_{\vec{q}}^{j}+\frac{q^{i} q^{j}}{\vec{q}^{2}} P_{\vec{q}}^{j} \equiv P_{\vec{q}}^{\mathrm{T} i}+P_{\vec{q}}^{\mathrm{L} i}
$$

We note that here is the difference from the Heisenberg antiferromagnet in which the momentum is absent. In the Heisenberg antiferromagnet, the slow mode for the energy density is diffusive, while the chiral system gives the propagating mode for the energy because of the presence of the momentum density. What is this propagating mode? It would be essentially a sound wave, but not exactly the same as the usual first sound of the normal fluid. For comparison, consider the slow modes in the normal fluid $[17,25,26]$, in which the slow variables are the particle number density $N_{\vec{q}}$, the energy density $E_{\vec{q}}$, and the velocity $u_{\vec{q}}^{i}$. The $N_{\vec{q}}$ and $E_{\vec{q}}$ may be transformed to the entropy density $S_{\vec{q}}$ and the pressure $p_{\vec{q}}$ through

$$
\begin{aligned}
& S_{\vec{q}}=\frac{1}{T}\left(E_{\vec{q}}-m h N_{\vec{q}}\right), \\
& p_{\vec{q}}=\frac{m}{\rho \chi_{s}(\vec{q})} N_{\vec{q}}-\frac{1}{\alpha_{s}(\vec{q})} S_{\vec{q}},
\end{aligned}
$$

where $\rho$ and $h$ are the equilibrium values of the density and the enthalpy per unit mass, $m$ is the mass of a molecule, and $\chi_{s}(\vec{q})$ and $\alpha_{s}(\vec{q})$ are the $\vec{q}$-dependent adiabatic compressibility and adiabatic thermal expansion coefficients. The kinetic equation shows that the modes for $S_{\vec{q}}$ and $u_{\vec{q}}^{\mathrm{T}}$ are diffusive while $p_{\vec{q}}$ and $u_{\vec{q}}^{\mathrm{L}}$ combine to give the first sound mode. See Table 1 . Thus our propagating mode is to be compared with the first sound in the normal fluid. If the first sound is called the pressure wave, our propagating mode may be called the energy wave mode. If it is allowed to identify the chiral charge in the chiral system with the number density in the normal fluid, we can say that the basis that diagonalizes the equation in each system is different. The basis of the one system can be obtained by the linear transformation from that of the other system.

Now we move to the ordered phase. The frequency matrix in the ordered phase 
is found to be

$$
\omega_{j l}=\left(\begin{array}{ccccccc}
-\frac{k_{\mathrm{B}} T}{2 \omega_{1 \vec{q}} \chi_{1 \vec{q}}} & 0 & 0 & 0 & 0 & \frac{-B}{\chi_{e \vec{q}}} & 0 \\
0 & \frac{k_{\mathrm{B}} T}{2 \omega_{1 \vec{q}} \chi_{1 \vec{q}}} & 0 & 0 & 0 & \frac{B^{*}}{\chi_{e \vec{q}}} & 0 \\
0 & 0 & -\frac{k_{\mathrm{B}} T}{2 \omega_{2 \vec{q}} \chi_{2 \vec{q}}} & 0 & \frac{A}{\chi_{Q \vec{q}}} & 0 & 0 \\
0 & 0 & 0 & \frac{k_{\mathrm{B}} T}{2 \omega_{2 \vec{q}} \chi_{2 \vec{q}}} & \frac{-A^{*}}{\chi_{Q \vec{q}}} & 0 & 0 \\
0 & 0 & \frac{A^{*}}{\chi_{2 \vec{q}}} & \frac{-A}{\chi_{2 \vec{q}}} & 0 & 0 & 0 \\
\frac{-B^{*}}{\chi_{1 \vec{q}}} & \frac{B}{\chi_{1 \vec{q}}} & 0 & 0 & 0 & 0 & -\frac{D}{\chi_{p \vec{q}}} q^{i} \\
0 & 0 & 0 & 0 & 0 & -\frac{D}{\chi_{e q}} q^{i} & 0
\end{array}\right),
$$

where

$$
\begin{aligned}
& A=k_{\mathrm{B}} T \frac{\mathrm{i}}{2 \sqrt{V}} \frac{1}{\sqrt{2 \omega_{2 \overrightarrow{0}}}}\left\langle a_{1 \overrightarrow{0}}+a_{1 \overrightarrow{0}}^{\dagger}\right\rangle, \\
& B=k_{\mathrm{B}} T \frac{1}{2 \sqrt{V}} \frac{1}{\sqrt{2 \omega_{1 \vec{q}}}} \mu^{2} \frac{1}{\sqrt{\omega_{1 \vec{q}} \omega_{1 \overrightarrow{0}}}}\left\langle a_{1 \overrightarrow{0}}+a_{1 \overrightarrow{0}}^{\dagger}\right\rangle \\
& +k_{\mathrm{B}} T \frac{\lambda}{4} \frac{1}{V^{\frac{3}{2}}} \frac{1}{\sqrt{2 \omega_{1 \vec{q}}}} \sum_{\vec{k}_{1} \ldots \vec{k}_{4}} \delta_{\vec{q},-\vec{k}_{1}} \delta_{-\vec{q}, \vec{k}_{1} \cdots \vec{k}_{4}} \frac{1}{\sqrt{\omega_{1 \vec{k}_{1}} \omega_{1 \vec{k}_{2}} \omega_{b \vec{k}_{3}} \omega_{b \vec{k}_{4}}}} \\
& \times\left\langle\left(a_{1 \vec{k}_{2}}+a_{1-\vec{k}_{2}}^{\dagger}\right)\left(a_{b \vec{k}_{3}}+a_{b-\vec{k}_{3}}^{\dagger}\right)\left(a_{b \vec{k}_{4}}+a_{b-\vec{k}_{4}}^{\dagger}\right)\right\rangle .
\end{aligned}
$$

The $\phi_{1}$ mode, i.e., the sigma meson mode can be treated in the same way as in the disordered phase. The $\phi_{2}$ mode now couples to the chiral charge $Q$. We concentrate on the $3 \times 3$ matrix of $\left\{\phi_{2 \vec{q}}, \phi_{2-\vec{q}}^{\dagger}, Q_{\vec{q}}\right\}$. It can be diagonalized readily to give the eigenvalues

$$
\lambda=0, \pm \sqrt{\left(\frac{k_{\mathrm{B}} T}{2 \omega_{2 \vec{q}} \chi_{2 \vec{q}}}\right)^{2}+\frac{2|A|^{2}}{\chi_{2 \vec{q}} \chi_{Q \vec{q}}}} .
$$

The zero eigenvalue gives a diffusive mode. The resulting propagating mode should be regarded as the $\phi_{2}$ mode modified by the interaction or the coupling with the chiral charge. Thus we impose the consistency condition that the

Table 1

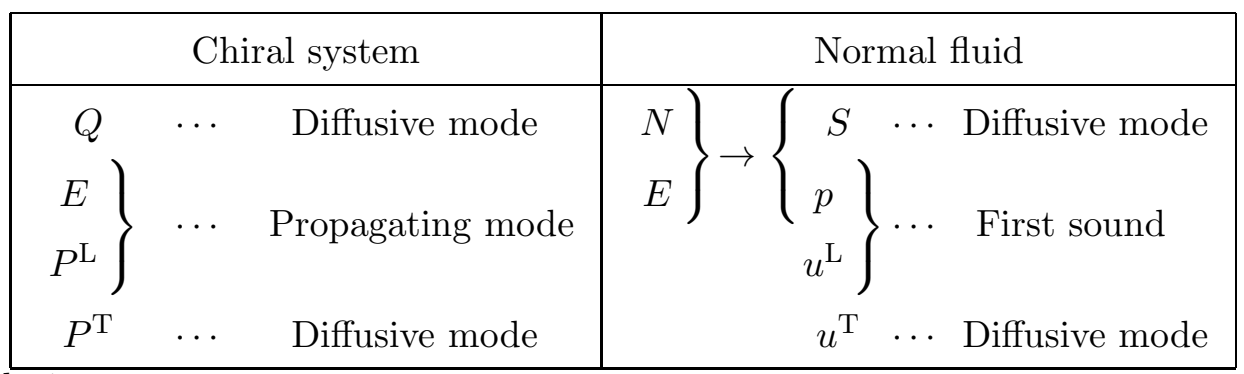

Comparison of the chiral system with the normal fluid. 
eigenvalue be identical to $\omega_{2 \vec{q}}$. Moreover since the $\phi_{2}$ mode must be the NambuGoldstone mode, we should have $\omega_{2 \vec{q}}=u q$, where $u$ is the pion velocity [8]. Thus we have

$$
\sqrt{\left(\frac{k_{\mathrm{B}} T}{2 \omega_{2 \vec{q}} \chi_{2 \vec{q}}}\right)^{2}+\frac{2|A|^{2}}{\chi_{2 \vec{q}} \chi_{Q \vec{q}}}}=\omega_{2 \vec{q}}=u q .
$$

Furthermore if we assume

$$
\frac{2 \chi_{2 \vec{q}}}{k_{\mathrm{B}} T}=\frac{Z_{\pi}}{\vec{q}^{2}},
$$

then in the approximation of $|\vec{q}| \ll 1$, we find

$$
\frac{1}{u^{2} Z_{\pi}^{2}}+\frac{4|A|_{\vec{q}=0}^{2}}{k_{\mathrm{B}} T \chi_{Q \vec{q}=0}} \frac{1}{Z_{\pi}}=u^{2},
$$

from which the renormalization constant $Z_{\pi}$ can be determined if $u$ is given. This treatment for the pion mode is essentially the same as that for the second sound mode in the superfluid transition [16].

We can see that in the ordered phase, the modes of the energy and momentum couple to the sigma meson mode through the nondiagonal elements.

For summary, let us compare the ordered phase with the superfluid [16,26,27]. See Table 2. In the superfluid, the order parameter $\left(\Phi, \Phi^{\dagger}\right)$ and the entropy $S$ constitute the second sound wave mode and the diffusive mode. If we call the second sound the entropy wave, the $\pi$ mode may be viewed as the chiral charge wave in the analogy with the superfluid.

Finally, we consider the hydrodynamic modes of the chiral system away from the critical point. In general, the conserved quantitiy and the transverse component of the order parameter in the ordered phase are slow variables even away from the critical point [26], the latter of which is slow because of a dynamical reason rather than a kinematical one. The fluctuations of those variables make up the slow (or hydrodynamic) modes and appear in the spectral functions as narrow peaks though their widths are finite for the finite wavelength

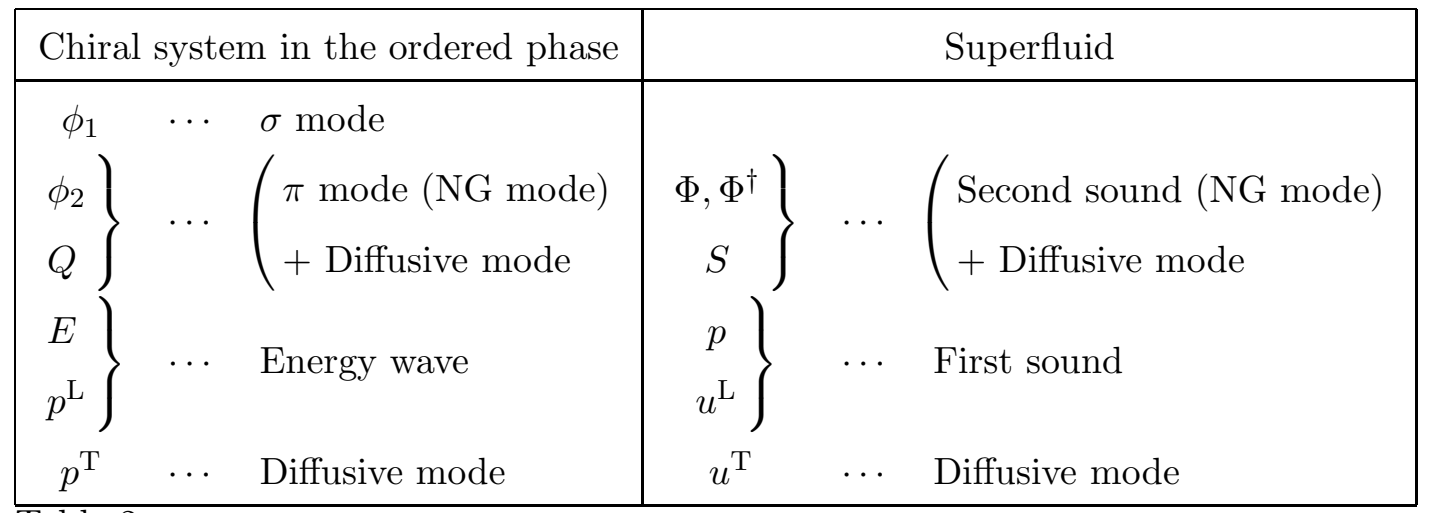

Table 2

Comparison of the ordered phase of the chiral system with the superfluid. 
fluctuations. In the chiral system, the conserved quantities, $Q, E$ and $P^{i}$, are the slow variables, so that the slow modes found in the above consideration will be responsible for the hydrodynamics of the system. In the disordered phase, since the meson fields $\phi_{1}$ and $\phi_{2}$ are not conserved quantities, the fluctuations of $\phi_{1}$ and $\phi_{2}$ do not make hydrodynamic mode and decay (or relax) in the microscopic time scale. Thus the $\sigma$ and $\pi$ meson modes will disappear away from the critical point [6]. In the ordered phase, $\phi_{2}$ being the transverse fluctuation of the order parameter is the dynamically slow variable. Thus the $\pi$ meson mode appears as a hydrodynamic mode. The $\sigma$ meson mode, on the other hand, is not a hydrodynamic mode and decays in the microscopic time scale. This is, of course, consistent with the fact that we can not observe the sigma meson as a narrow peak in the vacuum.

\section{Kinetic equation for the chiral phase transition above $T_{\mathbf{c}}$ in the $\mathrm{O}(4)$ linear sigma model}

In this section, we give the kinetic equation for the $\mathrm{O}(4)$ linear sigma model. For simplicity, we consider the disordered phase. The emphasis will be put on the effect of the propagating mode of the order parameter, which is replaced by the diffusive mode the itinerant antiferromagnet.

The Lagrangian of the $\mathrm{O}(4)$ linear sigma model is given by

$$
\mathcal{L}=\frac{1}{2}\left(\partial_{\mu} \phi_{\alpha}\right)^{2}-\frac{1}{2} \mu^{2} \phi_{\alpha}{ }^{2}-\frac{\lambda}{4}\left(\phi_{\alpha}{ }^{2}\right)^{2}
$$

where $\alpha$ runs from 0 to 3 and $\phi_{\alpha}$ denotes the meson fields, $\phi_{\alpha}=\left(\phi_{0}, \phi_{1}, \phi_{2}, \phi_{3}\right)$. For the pion field, we may use $\phi_{a}=\left(\phi_{1}, \phi_{2}, \phi_{3}\right)$ with $a$ running from 1 to 3 . The conserved currents for the chiral $\mathrm{SU}_{\mathrm{L}}(2) \otimes \mathrm{SU}_{\mathrm{R}}(2)$ transformation are the vector and axial vector currents given by

$$
\begin{aligned}
& V_{a}^{\mu}=\epsilon_{a b c} \phi_{b} \partial^{\mu} \phi_{c} \\
& A_{a}^{\mu}=\phi_{a} \partial^{\mu} \phi_{0}-\phi_{0} \partial^{\mu} \phi_{a}
\end{aligned}
$$

where $\epsilon_{a b c}$ is the total antisymmetric tensor with $\epsilon_{123}=1$. The chiral charges are then

$$
\begin{aligned}
Q_{a}^{V}(t) & =\int \mathrm{d}^{3} x V_{a}^{0}(\vec{x}, t)=\int \mathrm{d}^{3} x \epsilon_{a b c} \phi_{b} \partial_{t} \phi_{c}, \\
Q_{a}^{A}(t) & =\int \mathrm{d}^{3} x A_{a}^{0}(\vec{x}, t)=\int \mathrm{d}^{3} x\left(\phi_{a} \partial_{t} \phi_{0}-\phi_{0} \partial_{t} \phi_{a}\right),
\end{aligned}
$$

which may be accommodated in the antisymmetric tensor $Q_{\alpha \beta}(t)$, i.e.,

$$
Q_{\alpha \beta}(t)=\int \mathrm{d}^{3} x \epsilon_{\alpha \beta \gamma \delta} \phi_{\gamma} \partial_{t} \phi_{\delta}
$$


where $\epsilon_{\alpha \beta \gamma \delta}$ is the total antisymmetric tensor with $\epsilon_{0123}=1$. The $Q_{a}^{V}$ and $Q_{a}^{A}$ are related to $Q_{\alpha \beta}$ as

$$
Q_{0 a}=Q_{a}^{V}, \quad Q_{a b}=-\epsilon_{a b c} Q_{c}^{A}
$$

The meson field $\phi_{\alpha}$ and its conjugate momentum $\pi_{\alpha}$ are expanded in terms of the creation and annihilation operators,

$$
\begin{aligned}
& \phi_{\alpha}(\vec{x}, t)=\frac{1}{\sqrt{V}} \sum_{\vec{k}} \frac{1}{\sqrt{2 \omega_{\vec{k}}}}\left(a_{\alpha \vec{k}}(t)+a_{\alpha-\vec{k}}^{\dagger}(t)\right) e^{\mathrm{i} \vec{k} \cdot \vec{x}}, \\
& \pi_{\alpha}(\vec{x}, t)=\frac{1}{\sqrt{V}} \sum_{\vec{k}}(-\mathrm{i}) \sqrt{\frac{\omega_{\vec{k}}}{2}}\left(a_{\alpha \vec{k}}(t)-a_{\alpha-\vec{k}}^{\dagger}(t)\right) e^{\mathrm{i} \vec{k} \cdot \vec{x}} .
\end{aligned}
$$

We note that the dispersion $\omega_{\vec{k}}$ does not depend on $\alpha$ because all the $\sigma$ and $\pi$ mesons are degenerated in the disordered phase. We also note that the six charges, $Q_{a}^{V}$ and $Q_{a}^{A}$, are degenerated in this phase.

The quantization conditions

$$
\begin{aligned}
& {\left[\phi_{\alpha}(\vec{x}, t), \pi_{\beta}(\vec{y}, t)\right]=\mathrm{i} \delta_{\alpha \beta} \delta(\vec{x}-\vec{y}),} \\
& {\left[\phi_{\alpha}(\vec{x}, t), \phi_{\beta}(\vec{y}, t)\right]=\left[\pi_{\alpha}(\vec{x}, t), \pi_{\beta}(\vec{y}, t)\right]=0,}
\end{aligned}
$$

or

$$
\begin{aligned}
& {\left[a_{\alpha \vec{k}}(t), a_{\beta \vec{k}^{\prime}}^{\dagger}(t)\right]=\delta_{\alpha \beta} \delta_{\vec{k} \vec{k}^{\prime}},} \\
& {\left[a_{\alpha \vec{k}}(t), a_{\beta \vec{k}^{\prime}}(t)\right]=\left[a_{\alpha \vec{k}}^{\dagger}(t), a_{\beta \vec{k}^{\prime}}^{\dagger}(t)\right]=0}
\end{aligned}
$$

provide us with the rule to calculate the coefficients in the kinetic equation.

The slow variables that consist of the Fourier components of the order parameter and its conjugate, the chiral charges, the energy density and the momentum 
density are defined by

$$
\begin{aligned}
\phi_{\alpha \vec{q}}(t) & =\frac{1}{\sqrt{2 \omega_{\vec{q}}}} a_{\alpha \vec{q}}(t), \\
\phi_{\alpha-\vec{q}}^{\dagger}(t) & =\frac{1}{\sqrt{2 \omega_{\vec{q}}}} a_{\alpha-\vec{q}}^{\dagger}(t), \\
Q_{\alpha \beta \vec{q}}(t) & =\epsilon_{\alpha \beta \gamma \delta} \frac{1}{\sqrt{V}} \int \mathrm{d}^{3} x e^{-\mathrm{i} \vec{q} \cdot \vec{x}} \phi_{\gamma} \pi_{\delta}(\vec{x}, t), \\
E_{\vec{q}}(t) & =\frac{1}{\sqrt{V}} \int \mathrm{d}^{3} x e^{-\mathrm{i} \vec{q} \cdot \vec{x}}\left[\left\{\frac{1}{2} \pi_{\alpha}{ }^{2}+\frac{1}{2}\left(\vec{\nabla} \phi_{\alpha}\right)^{2}+\frac{1}{2} \mu^{2} \phi_{\alpha}{ }^{2}\right.\right. \\
& \left.\left.\quad+\frac{\lambda}{4}\left(\phi_{\alpha}{ }^{2}\right)^{2}\right\}(\vec{x}, t)-\langle\text { the same expression }\rangle_{\mathrm{eq}}\right], \\
P_{\vec{q}}^{i}(t) & =\frac{1}{\sqrt{V}} \int \mathrm{d}^{3} x e^{-\mathrm{i} \vec{q} \cdot \vec{x}}\left(-\pi_{\alpha} \nabla^{i} \phi_{\alpha}\right)(\vec{x}, t) .
\end{aligned}
$$

In the same way as Eq. (5.7), we can obtain $Q_{a \vec{q}}^{V}$ and $Q_{a \vec{q}}^{A}$ from $Q_{\alpha \beta \vec{q}}$ by

$$
Q_{0 a \vec{q}}=Q_{a \vec{q}}^{V}, \quad Q_{a b \vec{q}}=-\epsilon_{a b c} Q_{c \vec{q}}^{A} .
$$

The $Q_{\alpha \beta \vec{q}}, E_{\vec{q}}$ and $P_{\vec{q}}^{i}$ may be written in terms of the creation and annihilation operators as

$$
\begin{aligned}
Q_{\alpha \beta \vec{q}}(t)= & \epsilon_{\alpha \beta \gamma \delta} \frac{1}{\sqrt{V}} \sum_{\vec{k}} \frac{-\mathrm{i}}{2} \sqrt{\frac{\omega_{\vec{q}-\vec{k}}}{\omega_{\vec{k}}}}\left(a_{\gamma \vec{k}}+a_{\gamma-\vec{k}}^{\dagger}\right)\left(a_{\delta \vec{q}-\vec{k}}-a_{\delta \vec{k}-\vec{q}}^{\dagger}\right), \\
E_{\vec{q}}(t)= & \frac{1}{2} \frac{1}{\sqrt{V}} \sum_{\vec{k}} \frac{-1}{2} \sqrt{\omega_{\vec{k}} \omega_{\vec{q}-\vec{k}}}\left(a_{\alpha \vec{k}}-a_{\alpha-\vec{k}}^{\dagger}\right)\left(a_{\alpha \vec{q}-\vec{k}}-a_{\alpha \vec{k}-\vec{q}}^{\dagger}\right) \\
& +\frac{1}{2} \frac{1}{\sqrt{V}} \sum_{\vec{k}}\left[-\vec{k} \cdot(\vec{q}-\vec{k})+\mu^{2}\right] \frac{1}{2} \frac{1}{\sqrt{\omega_{\vec{k}} \omega_{\vec{q}-\vec{k}}}}\left(a_{\alpha \vec{k}}+a_{\alpha-\vec{k}}^{\dagger}\right)\left(a_{\alpha \vec{q}-\vec{k}}+a_{\alpha \vec{k}-\vec{q}}^{\dagger}\right) \\
& +\frac{\lambda}{4} \frac{1}{V \frac{3}{2}} \sum_{\overrightarrow{k_{1} \cdots k_{4}}} \frac{1}{4} \delta_{\vec{q} \overrightarrow{k_{1}}+\cdots+\overrightarrow{k_{4}}} \frac{1}{\sqrt{\omega_{\overrightarrow{k_{1}}} \omega_{\overrightarrow{k_{2}}} \omega_{\overrightarrow{k_{3}}} \omega_{\overrightarrow{k_{4}}}}} \\
& \times\left(a_{\alpha \overrightarrow{k_{1}}}+a_{\alpha-\overrightarrow{k_{1}}}^{\dagger}\right)\left(a_{\alpha \overrightarrow{k_{2}}}+a_{\alpha-\overrightarrow{k_{2}}}^{\dagger}\right)\left(a_{\beta \overrightarrow{k_{3}}}+a_{\beta-\overrightarrow{k_{3}}}^{\dagger}\right)\left(a_{\beta \overrightarrow{k_{4}}}+a_{\beta-\overrightarrow{k_{4}}}^{\dagger}\right) \\
P_{\vec{q}}^{i}(t)= & -\frac{1}{\sqrt{V}} \sum_{\vec{k}} \frac{1}{2} \sqrt{\frac{\omega_{\vec{q}-\vec{k}}}{\omega_{\vec{k}}}} k^{i}\left(a_{\alpha \vec{q}-\vec{k}}-a_{\alpha \vec{k}-\vec{q}}^{\dagger}\right)\left(a_{\alpha \vec{k}}+a_{\alpha-\vec{k}}^{\dagger}\right) .
\end{aligned}
$$

We shall now derive the kinetic equation for each variable, the general form of which is given in Eqs. (3.1), (3.2) and (3.3).

We note that the second term in the expression of $\Omega_{j ; l m}$ involves the frequency $\omega_{\vec{q}}^{j p}$ and the presence of the propagating mode leads to appearance of the second term. Moreover the first term in $\Omega_{j ; l m}$ is also influenced by the presence of the propagating mode. Thus we expect a substantial difference in the kinetic 
equation between the chiral phase transition and the itinerant antiferromagnet because the propagating mode associated with the order parameter is absent in the latter.

The susceptibilities are defined by

$$
\begin{aligned}
& \chi_{\alpha \vec{q}} \equiv\left(\phi_{\alpha \vec{q}},\left(\phi_{\alpha \vec{q}}\right)^{\dagger}\right) \equiv \chi_{\phi \vec{q}}, \\
& \chi_{\alpha^{\dagger} \vec{q}} \equiv\left(\phi_{\alpha-\vec{q}}^{\dagger},\left(\phi_{\alpha-\vec{q}}^{\dagger}\right)^{\dagger}\right)=\chi_{\phi \vec{q}}, \\
& \chi_{Q_{\alpha \beta} Q_{\alpha \beta} \vec{q}} \equiv\left(Q_{\alpha \beta \vec{q}},\left(Q_{\alpha \beta \vec{q}}\right)^{\dagger}\right) \equiv \chi_{Q \vec{q}}, \\
& \chi_{e \vec{q}} \equiv\left(E_{\vec{q}},\left(E_{\vec{q}}\right)^{\dagger}\right) \equiv k_{\mathrm{B}} T^{2} C_{\vec{q}}, \\
& \chi_{P^{i} P^{i} \vec{q}} \equiv\left(P_{\vec{q}}^{i},\left(P_{\vec{q}}^{i}\right)^{\dagger}\right) \equiv \chi_{p \vec{q}},
\end{aligned}
$$

where $C_{\vec{q}}$ is the $\vec{q}$-dependent heat capacity. Note that the susceptibilities are not dependent on the indices $\alpha$ or $i=x, y, z$.

To obtain $\omega_{\vec{q}}^{j l}$ and $\Omega_{j ; l m}$, we need the commutation relations for the variables, which will be listed in Appendix A.

Now we write down the kinetic equation for each slow variable.

- Order parameter - $\phi_{\alpha \vec{q}}$

$$
\begin{aligned}
& \frac{\mathrm{d}}{\mathrm{d} t} \phi_{\alpha \vec{q}}=\left(-\mathrm{i} \omega_{\vec{q}}-\frac{L_{\phi}^{0}}{2 \chi_{\phi \vec{q}}}\right) \phi_{\alpha \vec{q}} \\
& \quad+\mathrm{i} \sum_{\vec{k}}\left[\frac{1}{2} \sum_{\beta} \sum_{\alpha^{\prime} \beta^{\prime}} \mathcal{V}\left(\begin{array}{l}
\alpha, \beta, Q_{\alpha^{\prime} \beta^{\prime}} \\
\vec{q}, \vec{k}, \vec{q}-\vec{k}
\end{array}\right) \phi_{\beta \vec{k}} Q_{\alpha^{\prime} \beta^{\prime} \vec{q}-\vec{k}}+\mathcal{V}\left(\begin{array}{l}
\alpha, \alpha, e \\
\vec{q}, \vec{k}, \vec{q}-\vec{k}
\end{array}\right) \phi_{\alpha \vec{k}} E_{\vec{q}-\vec{k}}\right. \\
& \quad+\sum_{i} \mathcal{V}\left(\begin{array}{l}
\alpha, \alpha, P^{i}, \vec{k}, \vec{q}-\vec{k} \\
)
\end{array}\right) \phi_{\alpha \vec{k}} P_{\vec{q}-\vec{k}}^{i}+\frac{1}{2} \sum_{\beta} \sum_{\alpha^{\prime} \beta^{\prime}} \mathcal{V}\left(\begin{array}{l}
\alpha, \beta^{\dagger}, Q_{\alpha^{\prime} \beta^{\prime}} \\
\vec{q}, \vec{k}, \vec{q}-\vec{k}
\end{array}\right) \phi_{\beta-\vec{k}}^{\dagger} Q_{\alpha^{\prime} \beta^{\prime} \vec{q}-\vec{k}} \\
& \left.\quad+\mathcal{V}\left(\begin{array}{l}
\alpha, \alpha^{\dagger}, e \\
\vec{q}, \vec{k}, \vec{q}-\vec{k}
\end{array}\right) \phi_{\alpha-\vec{k}}^{\dagger} E_{\vec{q}-\vec{k}}+\sum_{i} \mathcal{V}\left(\begin{array}{l}
\alpha, \alpha^{\dagger}, P^{i}, \vec{k}, \vec{q}-\vec{k} \\
\vec{q}
\end{array}\right) \phi_{\alpha-\vec{k}}^{\dagger} P_{\vec{q}-\vec{k}}^{i}\right]+f_{\vec{q}}^{\phi}
\end{aligned}
$$

where the dispersion $\omega_{\vec{q}}$ is given by $\omega_{\vec{q}}=Z^{-\frac{1}{2}} \sqrt{\vec{q}^{2}+m^{2}}$ in the Ornstein-Zernike approximation. We have defined the 'bare' Onsager coefficient $L_{\phi}^{0}$ for $\phi_{\alpha \vec{q}}$ and $\phi_{\alpha-\vec{q}}^{\dagger}$ by

$$
k_{\mathrm{B}} L_{\alpha \vec{q}, \alpha \vec{q}}^{0} / \chi_{\alpha \vec{q}}=k_{\mathrm{B}} L_{\alpha^{\dagger} \vec{q}, \alpha^{\dagger} \vec{q}}^{0} / \chi_{\alpha^{\dagger} \vec{q}}=L_{\phi}^{0} / 2 \chi_{\phi \vec{q}}
$$


The coefficient of the nonlinear terms, $\mathcal{V}_{j, l m} \equiv \Omega_{j ; l m} /\left(\chi_{l} \chi_{m}\right)^{\frac{1}{2}}$, are given by

$$
\begin{aligned}
& \mathcal{V}\left(\begin{array}{l}
\alpha, \beta, Q_{\alpha^{\prime} \beta^{\prime}} \\
\vec{q}, \vec{k}, \vec{q}-\vec{k}
\end{array}\right) \\
& =-k_{\mathrm{B}} T\left\{\frac{-\mathrm{i}}{2 \sqrt{V}} \epsilon_{\alpha \beta \alpha^{\prime} \beta^{\prime}} \sqrt{\frac{\omega_{\vec{k}}}{\omega_{\vec{q}}}}\left(\sqrt{\frac{\omega_{\vec{q}}}{\omega_{\vec{k}}}}+\sqrt{\frac{\omega_{\vec{k}}}{\omega_{\vec{q}}}}\right) \frac{1}{\chi_{Q \vec{q}-\vec{k}}}-\frac{\left(\phi_{\alpha \vec{q}}, \phi_{\beta \vec{k}}^{\dagger} Q_{\alpha^{\prime} \beta^{\prime} \vec{k}-\vec{q}}\right)}{2 \omega_{\vec{q}} \chi_{\phi \vec{q}} \chi_{\phi \vec{k}} \chi_{Q \vec{q}-\vec{k}}}\right\}, \\
& \mathcal{V}\left(\begin{array}{l}
\alpha, \beta, e \\
\vec{q}, \vec{k}, \vec{q}-\vec{k}
\end{array}\right)=-k_{\mathrm{B}} T\left\{\delta_{\alpha \beta} \frac{k_{\mathrm{B}} T}{\sqrt{V}} \frac{1}{2 \omega_{\vec{k}} \chi_{\phi \vec{k}} \chi_{e \vec{q}-\vec{k}}}-\frac{\left(\phi_{\alpha \vec{q}}, \phi_{\beta \vec{k}}^{\dagger} E_{\vec{k}-\vec{q}}\right)}{2 \omega_{\vec{q}} \chi_{\phi \vec{q}} \chi_{\phi \vec{k}} \chi_{e \vec{q}-\vec{k}}}\right\}, \\
& \mathcal{V}\left(\begin{array}{l}
\alpha, \beta, P^{i} \\
\vec{q}, \vec{k}, \vec{q}-\vec{k}
\end{array}\right) \\
& =-k_{\mathrm{B}} T\left\{\delta_{\alpha \beta} \frac{1}{2 \sqrt{V}} \sqrt{\frac{\omega_{\vec{k}}}{\omega_{\vec{q}}}}\left(\sqrt{\frac{\omega_{\vec{q}}}{\omega_{\vec{k}}}} k^{i}+\sqrt{\frac{\omega_{\vec{k}}}{\omega_{\vec{q}}}} q^{i}\right) \frac{1}{\chi_{p \vec{q}-\vec{k}}}-\frac{\left(\phi_{\alpha \vec{q}}, \phi_{\beta \vec{k}}^{\dagger} P_{\vec{k}-\vec{q}}^{i}\right)}{2 \omega_{\vec{q}} \chi_{\phi \vec{q}} \chi_{\phi \vec{k}} \chi_{p \vec{q}-\vec{k}}}\right\},
\end{aligned}
$$

$$
\begin{aligned}
& \mathcal{V}\left(\begin{array}{l}
\alpha, \beta^{\dagger}, Q_{\alpha^{\prime} \beta^{\prime}} \\
\vec{q}, \vec{k}, \vec{q}-\vec{k}
\end{array}\right) \\
& =-k_{\mathrm{B}} T\left\{\frac{-\mathrm{i}}{2 \sqrt{V}} \epsilon_{\alpha \beta \alpha^{\prime} \beta^{\prime}} \sqrt{\frac{\omega_{\vec{k}}}{\omega_{\vec{q}}}}\left(\sqrt{\frac{\omega_{\vec{q}}}{\omega_{\vec{k}}}}-\sqrt{\frac{\omega_{\vec{k}}}{\omega_{\vec{q}}}}\right) \frac{1}{\chi_{Q \vec{q}-\vec{k}}}-\frac{\left(\phi_{\alpha \vec{q}}, \phi_{\beta-\vec{k}} Q_{\alpha^{\prime} \beta^{\prime}-\vec{k}-\vec{q}}\right)}{2 \omega_{\vec{q}} \chi_{\phi \vec{q}} \chi_{\phi \vec{k}} \chi_{Q \vec{q}-\vec{k}}}\right\},
\end{aligned}
$$

$$
\mathcal{V}\left(\begin{array}{l}
\alpha, \beta^{\dagger}, e \\
\vec{q}, \vec{k}, \vec{q}-\vec{k}
\end{array}\right)=-k_{\mathrm{B}} T(-) \frac{\left(\phi_{\alpha \vec{q}}, \phi_{\beta-\vec{k}} E_{\vec{k}-\vec{q}}\right)}{2 \omega_{\vec{q}} \chi_{\phi \vec{q}} \chi_{\phi \vec{k}} \chi_{e \vec{q}-\vec{k}}}
$$

$$
\begin{aligned}
& \mathcal{V}\left(\begin{array}{l}
\alpha, \beta^{\dagger}, P^{i} \\
\vec{q}, \vec{k}, \vec{q}-\vec{k}
\end{array}\right) \\
& =-k_{\mathrm{B}} T\left\{\delta_{\alpha \beta} \frac{1}{2 \sqrt{V}} \sqrt{\frac{\omega_{\vec{k}}}{\omega_{\vec{q}}}}\left(\sqrt{\frac{\omega_{\vec{q}}}{\omega_{\vec{k}}}} k^{i}-\sqrt{\frac{\omega_{\vec{k}}}{\omega_{\vec{q}}}} q^{i}\right) \frac{1}{\chi_{p \vec{q}-\vec{k}}}-\frac{\left(\phi_{\alpha \vec{q}}, \phi_{\beta-\vec{k}} P_{\vec{k}-\vec{q}}^{i}\right)}{2 \omega_{\vec{q}} \chi_{\phi \vec{q}} \chi_{\phi \vec{k}} \chi_{p \vec{q}-\vec{k}}}\right\} .
\end{aligned}
$$


For illustration, we shall give an explicit derivation of $\mathcal{V}\left(\begin{array}{l}\alpha, \beta, e \\ \vec{q}, \vec{k}, \vec{q}-\vec{k}\end{array}\right)$ in Eq. (5.27).

$$
\begin{aligned}
& \mathcal{V}\left(\begin{array}{l}
\alpha, \beta, e \\
\vec{q}, \vec{k}, \vec{q}-\vec{k}
\end{array}\right) \\
& =\frac{-k_{\mathrm{B}} T}{\left(\chi_{\phi \vec{k}} \chi_{e \vec{q}-\vec{k}}\right)}\left\{\left\langle\left[\phi_{\alpha \vec{q}}, \phi_{\beta \vec{k}}^{\dagger} E_{\vec{q}-\vec{k}}^{\dagger}\right]\right\rangle-\left\langle\left[\phi_{\alpha \vec{q}},\left(\phi_{\alpha \vec{q}}\right)^{\dagger}\right]\right\rangle\left(\chi_{\phi \vec{q}}\right)^{-1}\left(\phi_{\alpha \vec{q}}, \phi_{\beta \vec{k}}^{\dagger} E_{\vec{q}-\vec{k}}^{\dagger}\right)\right\} \\
& =\frac{-k_{\mathrm{B}} T}{\left(\chi_{\phi \vec{k}} \chi_{e \vec{q}-\vec{k}}\right)}\left\{\left\langle\left[\phi_{\alpha \vec{q}}, \phi_{\beta \vec{k}}^{\dagger}\right] E_{\vec{q}-\vec{k}}^{\dagger}\right\rangle+\left\langle\phi_{\beta \vec{k}}^{\dagger}\left[\phi_{\alpha \vec{q}}, E_{\vec{q}-\vec{k}}^{\dagger}\right]\right\rangle-\frac{\left(\phi_{\alpha \vec{q}}, \phi_{\beta \vec{k}}^{\dagger} E_{\vec{k}-\vec{q}}\right)}{2 \omega_{\vec{q}} \chi_{\phi \vec{q}}}\right\} .
\end{aligned}
$$

The first term in the curly brackets vanishes. Note that the third term appears because of the presence of the propagating mode (the meson mode). For the second term, we use the approximation that

$$
\left[A_{\vec{k}}, B_{\vec{k}^{\prime}}\right] \cong\left[A_{\vec{k}+\vec{l}}, B_{\vec{k}^{\prime}-\vec{l}}\right]
$$

where $|\vec{k}|,\left|\vec{k}^{\prime}\right|,|\vec{l}| \ll 1$, which has a ground in the locality of the microscopic interaction [17]. With this approximation, we can manipulate the second term in Eq. (5.32);

$$
\begin{aligned}
& \text { (second term) }=\left\langle\phi_{\beta \vec{k}}^{\dagger}\left[\phi_{\alpha \vec{q}}, E_{\vec{k}-\vec{q}}\right]\right\rangle \\
& \qquad\left\langle\phi_{\beta \vec{k}}^{\dagger}\left[\phi_{\alpha \vec{k}}, E_{\overrightarrow{0}}\right]\right\rangle=\left\langle\phi_{\beta \vec{k}}^{\dagger}\left[\phi_{\alpha \vec{k}}, \frac{1}{\sqrt{V}} \mathcal{H}\right]\right\rangle \cong \frac{1}{\sqrt{V}}\left(\phi_{\beta \vec{k}}^{\dagger},\left[\phi_{\alpha \vec{k}}, \mathcal{H}\right]\right) \\
& =\frac{1}{\sqrt{V}}\left(\mathrm{i} \dot{\phi}_{\alpha \vec{k}}, \phi_{\beta \vec{k}}^{\dagger}\right)=\frac{1}{\sqrt{V}} k_{\mathrm{B}} T\left\langle\left[\phi_{\alpha \vec{k}}, \phi_{\beta \vec{k}}^{\dagger}\right]\right\rangle=\frac{1}{\sqrt{V}} k_{\mathrm{B}} T \delta_{\alpha \beta} \frac{1}{2 \omega_{\vec{k}}}
\end{aligned}
$$

where $\mathcal{H}$ is the Hamiltonian and we have used the Heisenberg equation $\mathrm{i} \dot{\mathcal{O}}=$ $[\mathcal{O}, \mathcal{H}]$ and the relation $\mathrm{i}(\dot{A}, B)=k_{\mathrm{B}} T\langle[A, B]\rangle$. Thus we obtain Eq. (5.27). We note that the second term of Eq. (5.32) is proportional to the frequency of the meson mode so that it vanishes if there is no propagating mode. Thus we see that the coefficient of the coupling of the order parameter and the energy density remains even in the disordered phase owing to the presence of the propagating mode of the order parameter. This is contrasted with the antiferromagnet, in which the mode of the order parameter is diffusive. In fact, in the antiferromagnet, the energy mode decouples from the other modes in the disordered phase as we have seen in Subsec. $3.2[16,17]$. We also note that the second term in Eq. (5.27) involving the three point correlation has arisen due to the propagating mode.

- Order parameter conjugate $-\phi_{\alpha-\vec{q}}^{\dagger}$ 
Similarly, we obtain the kinetic equation for $\phi_{\alpha-\vec{q}}^{\dagger}$.

$$
\begin{aligned}
& \frac{\mathrm{d}}{\mathrm{d} t} \phi_{\alpha-\vec{q}}^{\dagger}=\left(\mathrm{i} \omega_{\vec{q}}-\frac{L_{\phi}^{0}}{2 \chi_{\phi \vec{q}}}\right) \phi_{\alpha-\vec{q}}^{\dagger} \\
& +\mathrm{i} \sum_{\vec{k}}\left[\frac{1}{2} \sum_{\beta} \sum_{\alpha^{\prime} \beta^{\prime}} \mathcal{V}\left(\begin{array}{l}
\alpha^{\dagger}, \beta, Q_{\alpha^{\prime} \beta^{\prime}} \\
\vec{q}, \vec{k}, \vec{q}-\vec{k}
\end{array}\right) \phi_{\beta \vec{k}} Q_{\alpha^{\prime} \beta^{\prime} \vec{q}-\vec{k}}+\mathcal{V}\left(\begin{array}{c}
\alpha^{\dagger}, \alpha, e \\
\vec{q}, \vec{k}, \vec{q}-\vec{k}
\end{array}\right) \phi_{\alpha \vec{k}} E_{\vec{q}-\vec{k}}\right. \\
& +\sum_{i} \mathcal{V}\left(\begin{array}{l}
\alpha^{\dagger}, \alpha, P^{i}, \vec{k}, \vec{q}-\vec{k} \\
)
\end{array}\right) \phi_{\alpha \vec{k}} P_{\vec{q}-\vec{k}}^{i}+\frac{1}{2} \sum_{\beta} \sum_{\alpha^{\prime} \beta^{\prime}} \mathcal{V}\left(\begin{array}{l}
\alpha^{\dagger}, \beta^{\dagger}, Q_{\alpha^{\prime} \beta^{\prime}} \\
\vec{q}, \vec{k}, \vec{q}-\vec{k}
\end{array}\right) \phi_{\beta-\vec{k}}^{\dagger} Q_{\alpha^{\prime} \beta^{\prime} \vec{q}-\vec{k}} \\
& \left.+\mathcal{V}\left(\begin{array}{l}
\alpha^{\dagger}, \alpha^{\dagger}, e \\
\vec{q}, \vec{k}, \vec{q}-\vec{k}
\end{array}\right) \phi_{\alpha-\vec{k}}^{\dagger} E_{\vec{q}-\vec{k}}+\sum_{i} \mathcal{V}\left(\begin{array}{l}
\alpha^{\dagger}, \alpha^{\dagger}, P^{i}, \vec{k}, \vec{q}-\vec{k} \\
)
\end{array}\right) \phi_{\alpha-\vec{k}}^{\dagger} P_{\vec{q}-\vec{k}}^{i}\right]+f_{\vec{q}}^{\phi}
\end{aligned}
$$

with the coefficients

$$
\begin{aligned}
& \mathcal{V}\left(\begin{array}{l}
\alpha^{\dagger}, \beta, Q_{\alpha^{\prime} \beta^{\prime}} \\
\vec{q}, \vec{k}, \vec{q}-\vec{k}
\end{array}\right) \\
& =-k_{\mathrm{B}} T\left\{\frac{-\mathrm{i}}{2 \sqrt{V}} \epsilon_{\alpha \beta \alpha^{\prime} \beta^{\prime}} \sqrt{\frac{\omega_{\vec{k}}}{\omega_{\vec{q}}}}\left(\sqrt{\frac{\omega_{\vec{q}}}{\omega_{\vec{k}}}}-\sqrt{\frac{\omega_{\vec{k}}}{\omega_{\vec{q}}}}\right) \frac{1}{\chi_{Q \vec{q}-\vec{k}}}+\frac{\left(\phi_{\alpha-\vec{q}}^{\dagger}, \phi_{\beta \vec{k}}^{\dagger} Q_{\alpha^{\prime} \beta^{\prime}-\vec{k}-\vec{q}}\right)}{2 \omega_{\vec{q}} \chi_{\phi \vec{q}} \chi_{\phi \vec{k}} \chi_{Q \vec{q}-\vec{k}}}\right\}, \\
& \mathcal{V}\left(\begin{array}{c}
\alpha_{\vec{q},}^{\dagger}, \beta, e \\
\vec{k}, \vec{q}-\vec{k}
\end{array}\right)=-k_{\mathrm{B}} T \frac{\left(\phi_{\alpha-\vec{q}}^{\dagger}, \phi_{\beta \vec{k}}^{\dagger} E_{\vec{k}-\vec{q}}\right)}{2 \omega_{\vec{q}} \chi_{\phi \vec{q}} \chi_{\phi \vec{k}} \chi_{e \vec{q}-\vec{k}}}, \\
& \mathcal{V}\left(\begin{array}{l}
\alpha^{\dagger}, \beta, P^{i} \\
\vec{q}, \vec{k}, \vec{q}-\vec{k}
\end{array}\right) \\
& =-k_{\mathrm{B}} T\left\{\delta_{\alpha \beta} \frac{1}{2 \sqrt{V}} \sqrt{\frac{\omega_{\vec{k}}}{\omega_{\vec{q}}}}\left(\sqrt{\frac{\omega_{\vec{q}}}{\omega_{\vec{k}}}} k^{i}-\sqrt{\frac{\omega_{\vec{k}}}{\omega_{\vec{q}}}} q^{i}\right) \frac{1}{\chi_{p \vec{q}-\vec{k}}}+\frac{\left(\phi_{\alpha-\vec{q}}^{\dagger}, \phi_{\beta \vec{k}}^{\dagger} P_{\vec{k}-\vec{q}}^{i}\right)}{2 \omega_{\vec{q}} \chi_{\phi \vec{q}} \chi_{\phi \vec{k}} \chi_{p \vec{q}-\vec{k}}}\right\},
\end{aligned}
$$

$$
\begin{aligned}
& \mathcal{V}\left(\begin{array}{l}
\alpha^{\dagger}, \beta^{\dagger}, Q_{\alpha^{\prime} \beta^{\prime}} \\
\vec{q}, \vec{k}, \vec{q}-\vec{k}
\end{array}\right) \\
& =-k_{\mathrm{B}} T\left\{\frac{-\mathrm{i}}{2 \sqrt{V}} \epsilon_{\alpha \beta \alpha^{\prime} \beta^{\prime}} \sqrt{\frac{\omega_{\vec{k}}}{\omega_{\vec{q}}}}\left(\sqrt{\frac{\omega_{\vec{q}}}{\omega_{\vec{k}}}}+\sqrt{\frac{\omega_{\vec{k}}}{\omega_{\vec{q}}}}\right) \frac{1}{\chi_{Q \vec{q}-\vec{k}}}+\frac{\left(\phi_{\alpha-\vec{q}}^{\dagger}, \phi_{\beta-\vec{k}} Q_{\alpha^{\prime} \beta^{\prime} \vec{k}-\vec{q}}\right)}{2 \omega_{\vec{q}} \chi_{\phi \vec{q}} \chi_{\phi \vec{k}} \chi_{Q \vec{q}-\vec{k}}}\right\},
\end{aligned}
$$

$$
\mathcal{V}\left(\begin{array}{c}
\alpha^{\dagger}, \beta^{\dagger}, e \\
\vec{q}, \vec{k}, \vec{q}-\vec{k}
\end{array}\right)=-k_{\mathrm{B}} T\left\{-\delta_{\alpha \beta} \frac{k_{\mathrm{B}} T}{\sqrt{V}} \frac{1}{2 \omega_{\vec{k}} \chi_{\phi \vec{k}} \chi_{e \vec{q}-\vec{k}}}+\frac{\left(\phi_{\alpha-\vec{q}}^{\dagger}, \phi_{\beta-\vec{k}} E_{\vec{k}-\vec{q}}\right)}{2 \omega_{\vec{q}} \chi_{\phi \vec{q}} \chi_{\phi \vec{k}} \chi_{e \vec{q}-\vec{k}}}\right\}
$$




$$
\begin{aligned}
& \mathcal{V}\left(\begin{array}{l}
\alpha^{\dagger}, \beta^{\dagger}, P^{i} \\
\vec{q}, \vec{k}, \vec{q}-\vec{k}
\end{array}\right) \\
& =-k_{\mathrm{B}} T\left\{\delta_{\alpha \beta} \frac{1}{2 \sqrt{V}} \sqrt{\frac{\omega_{\vec{k}}}{\omega_{\vec{q}}}}\left(\sqrt{\frac{\omega_{\vec{q}}}{\omega_{\vec{k}}}} k^{i}+\sqrt{\frac{\omega_{\vec{k}}}{\omega_{\vec{q}}}} q^{i}\right) \frac{1}{\chi_{p \vec{q}-\vec{k}}}+\frac{\left(\phi_{\alpha-\vec{q}}^{\dagger}, \phi_{\beta-\vec{k}} P_{\vec{k}-\vec{q}}^{i}\right)}{2 \omega_{\vec{q}} \chi_{\phi \vec{q}} \chi_{\phi \vec{k}} \chi_{p \vec{q}-\vec{k}}}\right\} .
\end{aligned}
$$

- Chiral charges $-Q_{\alpha \beta \vec{q}}$

Since all the six charges should behave in the same manner, we take specifically $Q_{1 \vec{q}}^{V}=Q_{01 \vec{q}}$. Its kinetic equation is given by

$$
\begin{aligned}
\frac{\mathrm{d}}{\mathrm{d} t} Q_{1 \vec{q}}^{V}= & -q^{2} \frac{L_{Q}^{0}}{\chi_{Q \vec{q}}} Q_{1 \vec{q}}^{V} \\
& +\mathrm{i} \sum_{\vec{k}}\left[\mathcal{V}\left(\begin{array}{l}
Q_{01}, 2,3 \\
\vec{q}, \vec{k}, \vec{q}-\vec{k}
\end{array}\right) \phi_{2 \vec{k}} \phi_{3 \vec{q}-\vec{k}}+\mathcal{V}\left(\begin{array}{l}
Q_{01}, 2,3^{\dagger} \\
\vec{q}, \vec{k}, \vec{q}-\vec{k}
\end{array}\right) \phi_{2 \vec{k}} \phi_{3 \vec{k}-\vec{q}}^{\dagger}\right. \\
& +\mathcal{V}\left(\begin{array}{l}
Q_{01}, 2^{\dagger}, 3 \\
\vec{k}, \vec{q}-\vec{k}
\end{array}\right) \phi_{2-\vec{k}}^{\dagger} \phi_{3 \vec{q}-\vec{k}}+\mathcal{V}\left(\begin{array}{l}
Q_{01}, 2^{\dagger}, 3^{\dagger} \\
\vec{q}, \vec{k}, \vec{q}-\vec{k}
\end{array}\right) \phi_{2-\vec{k}}^{\dagger} \phi_{3 \vec{k}-\vec{q}}^{\dagger} \\
& +\mathcal{V}\left(\begin{array}{l}
Q_{01}, Q_{2}^{V}, Q_{3}^{V} \\
\vec{q}, \vec{k}, \vec{q}-\vec{k}
\end{array}\right) Q_{2 \vec{k}}^{V} Q_{3 \vec{q}-\vec{k}}^{V}+\mathcal{V}\left(\begin{array}{l}
Q_{01}, Q_{2}^{A}, Q_{3}^{A} \\
\vec{q}, \vec{k}, \vec{q}-\vec{k}
\end{array}\right) Q_{2 \vec{k}}^{A} Q_{3 \vec{q}-\vec{k}}^{A} \\
& \left.+\sum_{i} \mathcal{V}\left(\begin{array}{l}
Q_{01}, Q_{1}^{V}, P^{i} \\
\vec{q}, \vec{k}, \vec{q}-\vec{k}
\end{array}\right) Q_{1 \vec{k}}^{V} P_{\vec{q}-\vec{k}}^{i}\right]+f_{\vec{q}}^{Q}
\end{aligned}
$$

with the coefficients

$$
\begin{aligned}
& \mathcal{V}\left(\begin{array}{l}
Q_{01}, 2,3 \\
\vec{q}, \vec{k}, \vec{q}-\vec{k}
\end{array}\right)=\mathcal{V}\left(\begin{array}{l}
Q_{01}, 2^{\dagger}, 3^{\dagger} \\
\vec{q}, \vec{k}, \vec{q}-\vec{k}
\end{array}\right) \\
& =-k_{\mathrm{B}} T \frac{\mathrm{i}}{2 \sqrt{V}}\left(\sqrt{\frac{\omega_{\vec{k}}}{\omega_{\vec{q}-\vec{k}}}}-\sqrt{\frac{\omega_{\vec{q}-\vec{k}}}{\omega_{\vec{k}}}}\right)\left(\sqrt{\frac{\omega_{\vec{q}-\vec{k}}}{\omega_{\vec{k}}}} \frac{1}{\chi_{\phi \vec{k}}}+\sqrt{\frac{\omega_{\vec{k}}}{\omega_{\vec{q}-\vec{k}}}} \frac{1}{\chi_{\phi \vec{q}-\vec{k}}}\right) \\
& \mathcal{V}\left(\begin{array}{l}
Q_{01}, 2,3^{\dagger} \\
\vec{q}, \vec{k}, \vec{q}-\vec{k}
\end{array}\right)=\mathcal{V}\left(\begin{array}{l}
Q_{01}, 2^{\dagger}, 3 \\
\vec{q}, \vec{k}, \vec{q}-\vec{k}
\end{array}\right) \\
& =-k_{\mathrm{B}} T \frac{\mathrm{i}}{2 \sqrt{V}}\left(\sqrt{\frac{\omega_{\vec{k}}}{\omega_{\vec{q}-\vec{k}}}}+\sqrt{\frac{\omega_{\vec{q}-\vec{k}}}{\omega_{\vec{k}}}}\right)\left(\sqrt{\frac{\omega_{\vec{q}-\vec{k}}}{\omega_{\vec{k}}}} \frac{1}{\chi_{\phi \vec{k}}}-\sqrt{\frac{\omega_{\vec{k}}}{\omega_{\vec{q}-\vec{k}}}} \frac{1}{\chi_{\phi \vec{q}-\vec{k}}}\right) \\
& \mathcal{V}\left(\begin{array}{l}
Q_{01}, Q_{2}^{V}, Q_{3}^{V} \\
\vec{q}, \vec{k}, \vec{q}-\vec{k}
\end{array}\right)=\mathcal{V}\left(\begin{array}{l}
Q_{01}, Q_{2}^{A}, Q_{3}^{A} \\
\vec{q}, \vec{k}, \vec{q}-\vec{k}
\end{array}\right)=-k_{\mathrm{B}} T \frac{\mathrm{i}}{\sqrt{V}}\left(\frac{1}{\chi_{Q \vec{k}}}-\frac{1}{\chi_{Q \vec{q}-\vec{k}}}\right) \\
& \mathcal{V}\left(\begin{array}{l}
Q_{01}, Q_{1}^{V}, P^{i} \\
\vec{q}, \vec{k}, \vec{q}-\vec{k}
\end{array}\right)=-k_{\mathrm{B}} T \frac{1}{\sqrt{V}} q^{i} \frac{1}{\chi_{p \vec{q}-\vec{k}}} .
\end{aligned}
$$

We have defined the 'bare' Onsager coefficient $L_{Q}^{0}$ for the chiral charge by

$$
k_{\mathrm{B}} L_{Q_{\alpha \beta} \vec{q}, Q_{\alpha \beta} \vec{q}}^{0} / \chi_{Q_{\alpha \beta} \vec{q}}=q^{2} L_{Q}^{0} / \chi_{Q \vec{q}},
$$


which involves $q^{2}$ because the chiral charge is a conserved quantity.

- Energy - $E_{\vec{q}}$

$$
\begin{aligned}
\frac{\mathrm{d}}{\mathrm{d} t} E_{\vec{q}}= & \mathrm{i} \omega_{e p^{i} \vec{q}} P_{\vec{q}}^{i}-\lambda^{0}\left(\frac{q^{2}}{C_{\vec{q}}} E_{\vec{q}}-\frac{T}{\rho+p} \mathrm{i} q^{i} \frac{\partial P_{\vec{q}}^{i}}{\partial t}\right) \\
& +\frac{\mathrm{i}}{2} \sum_{\vec{k} \alpha} \mathcal{V}\left(\begin{array}{l}
e, \alpha, \alpha \\
\vec{q}, \vec{k}, \vec{q}-\vec{k}
\end{array}\right) \phi_{\alpha \vec{k}} \phi_{\alpha \vec{q}-\vec{k}} \\
& +\mathrm{i} \sum_{\vec{k} \alpha} \mathcal{V}\left(\begin{array}{l}
e, \alpha, \alpha^{\dagger} \\
\vec{q}, \vec{k}, \vec{q}-\vec{k}
\end{array}\right)\left(\phi_{\alpha \vec{k}} \phi_{\alpha \vec{k}-\vec{q}}^{\dagger}-\left\langle\phi_{\alpha \vec{k}} \phi_{\alpha \vec{k}-\vec{q}}^{\dagger}\right\rangle\right) \\
& +\frac{\mathrm{i}}{2} \sum_{\vec{k} \alpha} \mathcal{V}\left(\begin{array}{l}
e, \alpha^{\dagger}, \alpha^{\dagger} \\
\vec{q}, \vec{k}, \vec{q}-\vec{k}
\end{array}\right) \phi_{\alpha-\vec{k}}^{\dagger} \phi_{\alpha \vec{k}-\vec{q}}^{\dagger} \\
& +\mathrm{i} \sum_{\vec{k} i} \mathcal{V}\left(\begin{array}{l}
e, e, P^{i} \\
\vec{q}, \vec{k}, \vec{q}-\vec{k}
\end{array}\right) E_{\vec{k}} P_{\vec{q}-\vec{k}}^{i}+f_{\vec{q}}^{e}
\end{aligned}
$$

The $\rho$ and $p$ in the dissipation term are the proper energy density and the pressure in the equilibrium state respectively. The $\lambda^{0}$ is the 'bare' heat conductivity and $C_{\vec{q}}$ is the $\vec{q}$-dependent specific heat. The dissipation terms for the energy and the momentum are derived from the relativistic hydrodynamics. The derivation will be given in Appendix B.

The frequency is given by

$$
\omega_{e p^{i} \vec{q}}=-\Theta q^{i} / \chi_{p \vec{q}}
$$

and the coefficients are computed as

$$
\begin{aligned}
& \mathcal{V}\left(\begin{array}{l}
e, \alpha, \alpha \\
\vec{q}, \vec{k}, \vec{q}-\vec{k}
\end{array}\right)=k_{\mathrm{B}} T \Theta q^{i} \frac{\left(P_{\vec{q}}^{i}, \phi_{\alpha \vec{k}}^{\dagger} \phi_{\alpha \vec{q}-\vec{k}}^{\dagger}\right)}{\chi_{p \vec{q}} \chi_{\phi \vec{k}} \chi_{\phi \vec{q}-\vec{k}}}, \\
& \mathcal{V}\left(\begin{array}{l}
e, \alpha, \alpha^{\dagger} \\
\vec{q}, \vec{k}, \vec{q}-\vec{k}
\end{array}\right)=-k_{\mathrm{B}} T\left\{-\frac{k_{\mathrm{B}} T}{\sqrt{V}} \frac{1}{2 \omega_{\vec{k}}} \frac{1}{\chi_{\phi \vec{k}} \chi_{\phi \vec{q}-\vec{k}}}-\Theta q^{i} \frac{\left(P_{\vec{q}}^{i}, \phi_{\alpha \vec{k}}^{\dagger} \phi_{\alpha \vec{k}-\vec{q}}\right)}{\chi_{p \vec{q}} \chi_{\phi \vec{k}} \chi_{\phi \vec{q}-\vec{k}}}\right\} \\
& \mathcal{V}\left(\begin{array}{l}
e, \alpha^{\dagger}, \alpha^{\dagger} \\
\vec{q}, \vec{k}, \vec{q}-\vec{k}
\end{array}\right)=k_{\mathrm{B}} T \Theta q^{i} \frac{\left(P_{\vec{q}}^{i}, \phi_{\alpha-\vec{k}} \phi_{\alpha \vec{k}-\vec{q}}\right)}{\chi_{p \vec{q}} \chi_{\phi \vec{k}} \chi_{\phi \vec{q}-\vec{k}}}, \\
& \mathcal{V}\left(\begin{array}{l}
e, e, P^{i} \\
\vec{q}, \vec{k}, \vec{q}-\vec{k}
\end{array}\right)=-k_{\mathrm{B}} T\left\{\frac{1}{\sqrt{V}}(q+k)^{i} \frac{1}{\chi_{e \vec{k}}}-\frac{k_{\mathrm{B}} T}{\sqrt{V}} \Theta k^{i} \frac{1}{\chi_{e \vec{k}} \chi_{p \vec{q}-\vec{k}}}+\Theta q^{j} \frac{\left(P_{\vec{q}}^{j}, E_{-\vec{k}} P_{\vec{k}-\vec{q}}^{i}\right)}{\chi_{p \vec{q}} \chi_{e \vec{k}} \chi_{p \vec{q}-\vec{k}}}\right\},
\end{aligned}
$$

where

$$
\Theta \equiv \frac{8}{V} \sum_{\vec{k}}\left(\omega_{\vec{k}}^{2}+\frac{1}{3} \vec{k}^{2}\right) \chi_{\phi \vec{k}}
$$


- Momentum - $P_{\vec{q}}^{i}$

$$
\begin{aligned}
& \frac{\mathrm{d}}{\mathrm{d} t} P_{\vec{q}}^{i}=\mathrm{i} \omega_{p^{i} e \vec{q}} E_{\vec{q}}-\lambda^{0}\left(-\frac{\mathrm{i} q^{i}}{C_{\vec{q}}} \frac{\partial E_{\vec{q}}}{\partial t}-\frac{T}{\rho+p} \frac{\partial^{2} P_{\vec{q}}^{i}}{\partial t^{2}}\right) \\
& -\frac{\eta^{0}}{\rho+p}\left(q^{2} P_{\vec{q}}^{i}+\frac{1}{3} q^{i}\left(\vec{q} \cdot \vec{P}_{\vec{q}}\right)\right)-\frac{\zeta^{0}}{\rho+p} q^{i}\left(\vec{q} \cdot \vec{P}_{\vec{q}}\right)
\end{aligned}
$$

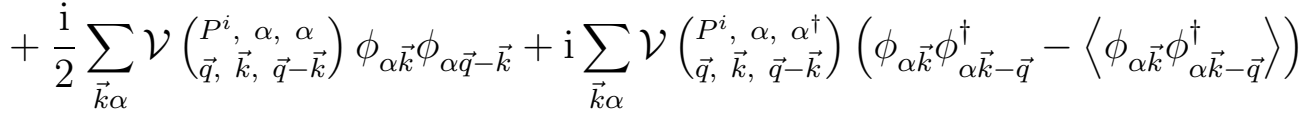

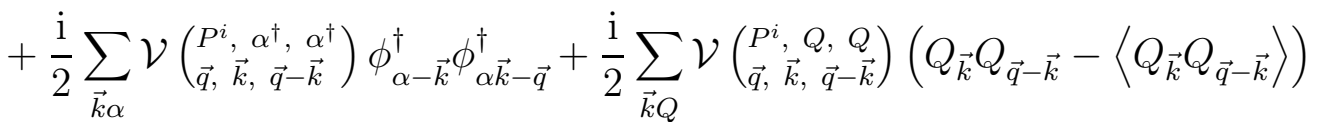

$$
\begin{aligned}
& +\frac{\mathrm{i}}{2} \sum_{\vec{k}} \mathcal{V}\left(\begin{array}{l}
P_{\vec{q}, \vec{k}}^{i}, e, e \\
\vec{q}-\vec{k}
\end{array}\right)\left(E_{\vec{k}} E_{\vec{q}-\vec{k}}-\left\langle E_{\vec{k}} E_{\vec{q}-\vec{k}}\right\rangle\right) \\
& +\frac{\mathrm{i}}{2} \sum_{\vec{k} j l} \mathcal{V}\left(\begin{array}{c}
P^{i}, P^{j}, \vec{k}, \vec{q}-\vec{k} \\
l
\end{array}\right)\left(P_{\vec{k}}^{j} P_{\vec{q}-\vec{k}}^{l}-\left\langle P_{\vec{k}}^{j} P_{\vec{q}-\vec{k}}^{l}\right\rangle\right)+f_{\vec{q}}^{p}
\end{aligned}
$$

The $\eta^{0}$ and $\zeta^{0}$ in the dissipation terms are the 'bare' shear and bulk viscosities. The dissipation terms are derived in Appendix B.

The frequency is given by

$$
\omega_{p^{i} e \vec{q}}=-\Theta q^{i} / \chi_{e \vec{q}},
$$

and the coefficients are computed likewise as

$$
\begin{aligned}
& \mathcal{V}\left(\begin{array}{l}
P^{i}, \alpha, \alpha \\
\vec{q}, \vec{k}, \vec{q}-\vec{k}
\end{array}\right)=-k_{\mathrm{B}} T\left[-\frac{1}{2 \sqrt{V}} \sqrt{\frac{\omega_{\vec{q}-\vec{k}}}{\omega_{\vec{k}}}}\left(\sqrt{\frac{\omega_{\vec{k}}}{\omega_{\vec{q}-\vec{k}}}}(q-k)^{i}+\sqrt{\frac{\omega_{\vec{q}-\vec{k}}}{\omega_{\vec{k}}}} k^{i}\right) \frac{1}{\chi_{\phi \vec{k}}}\right. \\
& \left.-\frac{1}{2 \sqrt{V}} \sqrt{\frac{\omega_{\vec{k}}}{\omega_{\vec{q}-\vec{k}}}}\left(\sqrt{\frac{\omega_{\vec{q}-\vec{k}}}{\omega_{\vec{k}}}} k^{i}+\sqrt{\frac{\omega_{\vec{k}}}{\omega_{\vec{q}-\vec{k}}}}(q-k)^{i}\right) \frac{1}{\chi_{\phi \vec{q}-\vec{k}}}-\Theta q^{i} \frac{\left(E_{\vec{q}}, \phi_{\alpha \vec{k}}^{\dagger} \phi_{\alpha \vec{q}-\vec{k}}^{\dagger}\right)}{\chi_{e \vec{q}} \chi_{\phi \vec{k}} \chi_{\phi \vec{q}-\vec{k}}}\right],
\end{aligned}
$$

$$
\begin{aligned}
& \mathcal{V}\left(\begin{array}{c}
P_{\vec{q}}^{i}, \alpha, \alpha, \alpha^{\dagger}, \vec{q}-\vec{k} \\
)
\end{array}\right)=-k_{\mathrm{B}} T\left[-\frac{1}{2 \sqrt{V}} \sqrt{\frac{\omega_{\vec{q}-\vec{k}}}{\omega_{\vec{k}}}}\left(\sqrt{\frac{\omega_{\vec{k}}}{\omega_{\vec{q}-\vec{k}}}}(q-k)^{i}-\sqrt{\frac{\omega_{\vec{q}-\vec{k}}}{\omega_{\vec{k}}}} k^{i}\right) \frac{1}{\chi_{\phi \vec{k}}}\right. \\
& \left.-\frac{1}{2 \sqrt{V}} \sqrt{\frac{\omega_{\vec{k}}}{\omega_{\vec{q}-\vec{k}}}}\left(\sqrt{\frac{\omega_{\vec{q}-\vec{k}}}{\omega_{\vec{k}}}} k^{i}-\sqrt{\frac{\omega_{\vec{k}}}{\omega_{\vec{q}-\vec{k}}}}(q-k)^{i}\right) \frac{1}{\chi_{\phi \vec{q}-\vec{k}}}-\Theta q^{i} \frac{\left(E_{\vec{q}}, \phi_{\alpha \vec{k}}^{\dagger} \phi_{\alpha \vec{k}-\vec{q}}\right)}{\chi_{e \vec{q}} \chi_{\phi \vec{k}} \chi_{\phi \vec{q}-\vec{k}}}\right],
\end{aligned}
$$




$$
\begin{aligned}
& \mathcal{V}\left(\begin{array}{l}
P^{i}, \alpha^{\dagger}, \alpha^{\dagger} \\
\vec{q}, \vec{k}, \vec{q}-\vec{k}
\end{array}\right)=-k_{\mathrm{B}} T\left[-\frac{1}{2 \sqrt{V}} \sqrt{\frac{\omega_{\vec{q}-\vec{k}}}{\omega_{\vec{k}}}}\left(\sqrt{\frac{\omega_{\vec{k}}}{\omega_{\vec{q}-\vec{k}}}}(q-k)^{i}+\sqrt{\frac{\omega_{\vec{q}-\vec{k}}}{\omega_{\vec{k}}}} k^{i}\right) \frac{1}{\chi_{\phi \vec{k}}}\right. \\
& \left.-\frac{1}{2 \sqrt{V}} \sqrt{\frac{\omega_{\vec{k}}}{\omega_{\vec{q}-\vec{k}}}}\left(\sqrt{\frac{\omega_{\vec{q}-\vec{k}}}{\omega_{\vec{k}}}} k^{i}+\sqrt{\frac{\omega_{\vec{k}}}{\omega_{\vec{q}-\vec{k}}}}(q-k)^{i}\right) \frac{1}{\chi_{\phi \vec{q}-\vec{k}}}-\Theta q^{i} \frac{\left(E_{\vec{q}}, \phi_{\alpha-\vec{k}} \phi_{\alpha \vec{k}-\vec{q}}\right)}{\chi_{e \vec{q}} \chi_{\phi \vec{k}} \chi_{\phi \vec{q}-\vec{k}}}\right), \\
& \mathcal{V}\left(\begin{array}{l}
P^{i}, Q, Q, Q \\
\vec{q}, \vec{k}, \vec{q}-\vec{k}
\end{array}\right)=-k_{\mathrm{B}} T\left[\frac{1}{\sqrt{V}}\left(k^{i} \frac{1}{\chi_{Q \vec{k}}}+(q-k)^{i} \frac{1}{\chi_{Q \vec{q}-\vec{k}}}\right)-\Theta q^{i} \frac{\left(E_{\vec{q}}, Q_{-\vec{k}} Q_{\vec{k}-\vec{q}}\right)}{\chi_{e \vec{q}} \chi_{Q \vec{k}} \chi_{Q \vec{q}-\vec{k}}}\right], \\
& \mathcal{V}\left(\begin{array}{l}
P^{i}, e, e \\
\vec{q}, \vec{k}, \vec{q}-\vec{k}
\end{array}\right)=-k_{\mathrm{B}} T \Theta q^{i}\left[\frac{k_{\mathrm{B}} T}{\sqrt{V}} \frac{1}{\chi_{e \vec{k}} \chi_{e \vec{q}-\vec{k}}}-\frac{\left(E_{\vec{q}}, E_{-\vec{k}} E_{\vec{k}-\vec{q}}\right)}{\chi_{e \vec{q}} \chi_{e \vec{k}} \chi_{E \vec{q}-\vec{k}}}\right], \\
& \mathcal{V}\left(\begin{array}{l}
P^{i}, P^{j}, P^{l} \\
\vec{q}, \vec{k}, \vec{q}-\vec{k}
\end{array}\right)=-k_{\mathrm{B}} T\left[\frac{1}{\sqrt{V}}\left\{\left(q^{j} \delta^{i l}+k^{i} \delta^{j l}\right) \frac{1}{\chi_{p \vec{k}}}+\left(q^{l} \delta^{i j}+(q-k)^{i} \delta^{j l}\right) \frac{1}{\chi_{p \vec{q}-\vec{k}}}\right\}\right. \\
& \left.-\Theta q^{i} \frac{\left(E_{\vec{q}}, P_{-\vec{k}}^{j} P_{\vec{k}-\vec{q}}^{l}\right)}{\chi_{e \vec{q}} \chi_{p \vec{k}} \chi_{p \vec{q}-\vec{k}}}\right] \text {. }
\end{aligned}
$$

We note that there are time derivatives in the dissipation terms for $E_{\vec{q}}$ and $P_{\vec{q}}^{i}$. These time derivatives may be eliminated by means of the kinetic equations themselves. We can perform it simply by replacing $\dot{E}_{\vec{q}}$ and $\dot{P}_{\vec{q}}^{i}$ with the associated frequency terms. This is because the time derivative of $E_{\vec{q}}$ and $P_{\vec{q}}^{i}$ and the frequency term are of second order with respect to the small quantities, that is, the wavenumber and the fluctuation of the slow variables, whereas the dissipation term and the nonlinear term are of higher order. Thus we have

$$
\begin{aligned}
\frac{\mathrm{d}}{\mathrm{d} t} E_{\vec{q}}= & -\mathrm{i} q^{j} \frac{\Theta}{\chi_{p \vec{q}}} P_{\vec{q}}^{j}-\frac{q^{2} \lambda^{0}}{C_{\vec{q}}}\left(1-\frac{T}{\rho+p} \frac{\Theta}{k_{\mathrm{B}} T^{2}}\right) E_{\vec{q}} \\
\frac{\mathrm{d}}{\mathrm{d} t} P_{\vec{q}}^{i}= & -\mathrm{i} q^{i} \frac{\Theta}{\chi_{e \vec{q}}} E_{\vec{q}}+\lambda^{0} \frac{q^{i}}{C_{\vec{q}}} \frac{\Theta}{\chi_{p \vec{q}}}\left(1-\frac{T}{\rho+p} \frac{\Theta}{k_{\mathrm{B}} T^{2}}\right)\left(\vec{q} \cdot \vec{P}_{\vec{q}}\right) \\
& -\frac{\eta^{0}}{\rho+p}\left(q^{2} P_{\vec{q}}^{i}+\frac{1}{3} q^{i}\left(\vec{q} \cdot \vec{P}_{\vec{q}}\right)\right)-\frac{\zeta^{0}}{\rho+p} q^{i}\left(\vec{q} \cdot \vec{P}_{\vec{q}}\right)
\end{aligned}
$$

where the dissipation term and the nonlinear term are omitted. 


\section{Dynamic critical exponents}

We have derived the kinetic equation for the chiral phase transition in the previous section. For the full analysis, the numerical calculation would be involved. As an application without the numerical work, we shall estimate the dynamic critical exponents from the kinetic equation in this section. The dynamic critical exponents specify the way how the typical inverse time scales of each slow mode, i.e., the widths and the frequencies are scaled. The fluctuation-dissipation theorem may allow us to imagine that the characteristic scaling law of the dynamic fluctuation of the slow variable originates from the characteristic behavior of the fluctuation in equilibrium states. Then it is reasonable to expect that there are some relations between the dynamic and static critical exponents. Actually, the dynamic exponents are given in terms of the static ones within the mode coupling theory as we will see below. We also discuss the anomaly of the Onsager coefficients, which are caused by renormalization from the nonlinear terms.

The analysis goes exactly in the same way as in Ref. [17]. In Sec. 6.1, we prepare the general formalism for obtaining the dynamic critical exponents. In Sec. 6.2, we apply the formalism to the chiral phase transition.

\subsection{General formalism}

In order to obtain the dynamic critical exponents, we have to know the characteristic scaling behavior of the dynamic fluctuations of the slow variables. In the mode coupling theory, the scaling properties of the dynamic fluctuations are given by those of the static ones as a starting point. The amplitude of the characteristic static fluctuation of the slow variable $A_{j}$ is measured by its static susceptibility $\chi_{j}=\left\langle\left|A_{j}\right|^{2}\right\rangle$. We define the "reduced" slow variable $\tilde{A}_{j}$ by

$$
\tilde{A}_{j}=A_{j} / \sqrt{\chi_{j}} .
$$

We also rescale the kinetic equation (3.1) to obtain the reduced kinetic equation for $\tilde{A}_{j}$,

$$
\frac{\mathrm{d}}{\mathrm{d} t} \tilde{A}_{j}(t)=\sum_{l}\left(\mathrm{i} \tilde{\omega}_{j l}-\frac{k_{\mathrm{B}} L_{j l}^{0}}{\sqrt{\chi_{j} \chi_{l}}}\right) \tilde{A}_{l}(t)+\frac{\mathrm{i}}{2} \sum_{l m} \tilde{\Omega}_{j ; l m}\left(\tilde{A}_{l} \tilde{A}_{m}-\left\langle\tilde{A}_{l} \tilde{A}_{m}\right\rangle\right)+\tilde{f}_{j},
$$


where

$$
\begin{aligned}
\tilde{\omega}_{j l} & \equiv-k_{\mathrm{B}} T\left\langle\left[\tilde{A}_{j}, \tilde{A}_{l}\right]\right\rangle=\sqrt{\frac{\chi_{l}}{\chi_{j}}} \omega_{j l} \\
\tilde{\Omega}_{j ; l m} & \equiv-k_{\mathrm{B}} T\left\{\left\langle\left[\tilde{A}_{j}, \tilde{A}_{l} \tilde{A}_{m}\right]\right\rangle-\sum_{p}\left\langle\left[\tilde{A}_{j}, \tilde{A}_{p}\right]\right\rangle\left(\tilde{A}_{p}, \tilde{A}_{l} \tilde{A}_{m}\right)\right\}=\Omega_{j ; l m} / \sqrt{\chi_{j}} \\
\tilde{f}_{j} & \equiv f_{j} / \sqrt{\chi_{j}}
\end{aligned}
$$

The reduced variables have a finite amplitude of order unity, so that the information of the characteristic scaling laws of the fluctuations of the slow variables is now pushed away into the coefficients of the reduced kinetic equation, which are given in Eqs. (6.3) and (6.4).

We consider the time correlation function for the reduced variable defined by

$$
g_{j l}(t)=\left\langle\tilde{A}_{j}(t) \tilde{A}_{l}(0)^{\dagger}\right\rangle, \quad(t \geq 0)
$$

which we will call simply the propagator. The physical information is contained in those propagators. The dynamics of the reduced variables is subject to the kinetic equation (6.2). By solving it, we can calculate the propagator.

In order to analyze the propagator, it is convenient to introduce the second quantized formalism, in which we can make the field theoretical techniques and considerations. In the second quantized formalism, the propagator is written as

$$
g_{j l}(t)=\left\langle 0\left|\alpha_{j} e^{-i t \mathcal{H}} \tilde{\alpha}_{l}\right| 0\right\rangle . \quad(t \geq 0)
$$

$\alpha_{j}$ and $\tilde{\alpha}_{j}$ are the operators that create and annihilate the mode $j$. We have defined the associated "vacuum" state $|0\rangle$, in which no $j$ modes are present. The "Hamiltonian" $\mathcal{H}$ governs the dynamics of the modes and is given by

$$
\begin{aligned}
\mathcal{H} & =\mathcal{H}_{0}+\mathcal{H}^{\prime}, \\
\mathcal{H}_{0} & =-\sum_{j l}\left[\tilde{\omega}_{j l}+\mathrm{i}\left(\chi_{j} \chi_{l}\right)^{-\frac{1}{2}} L_{j l}^{0}\right] \tilde{\alpha}_{j}^{0} \alpha_{l}^{0}, \\
\mathcal{H}^{\prime} & =-\frac{1}{2} \sum_{j l m}\left(\tilde{\Omega}_{j ; l m}^{*} \tilde{\alpha}_{l} \tilde{\alpha}_{m} \alpha_{j}+\tilde{\Omega}_{j ; l m} \tilde{\alpha}_{j} \alpha_{l} \alpha_{m}\right),
\end{aligned}
$$

where $\mathcal{H}_{0}$ and $\mathcal{H}^{\prime}$ are the free and interaction "Hamiltonians" and correspond to the linear and nonlinear terms in the kinetic equation respectively. We note that the free "Hamiltonian" is not Hermitian because of the dissipation term. In the following, we will use $\mathcal{H}_{0 j l}^{N}$ and $\mathcal{H}_{0 j l}^{D}$ to denote the two kinds of terms in Eq. (6.9), which describe non-dissipative and dissipative processes respectively. In the second quantized formalism, the expression of the propagator Eq. (6.7) can have the interpretation that the mode $l$ is created at $t=0$, which turns 
into the mode $j$ through the mixing and coupling with the other modes to be annihilated at a later time $t$.

We note that in this formalism, the reduced coefficients $\tilde{\omega}$ 's and $\tilde{\Omega}$ 's, which contain the information of the characteristic scaling properties of the fluctuations, appear in the "Hamiltonian" $\mathcal{H}$. Thus the "Hamiltonian" determines the scaling law of the slow modes.

Our present goal is to obtain the dynamic critical exponents or the characteristic time scales of each mode. For that purpose, it becomes necessary to consider each mode separately and derive the effective "Hamiltonians" for each mode. We can obtain the effective "Hamiltonian" by introducing the projection operator that separates the subspace of the interesting variables from that of the other variables. We denote the set of the slow variables for which we want the effective "Hamiltonian" by $\{a\}$ and the eliminated set by $\{b\}$. The "vacuum" state is then a product of the "vacuum" states for $\{a\}$ and $\{b\}$ denoted by $\left|0_{a}\right\rangle$ and $\left|0_{b}\right\rangle$,

$$
|0\rangle=\left|0_{a}\right\rangle\left|0_{b}\right\rangle
$$

The projection operator $\mathcal{P}_{b}$ is defined by $\mathcal{P}_{b}|\cdots\rangle=\left|0_{b}\right\rangle\left\langle 0_{b} \mid \cdots\right\rangle$, which projects arbitrary states of $\{b\}$ into the ground state $\left|0_{b}\right\rangle$.

Consider the one-sided Fourier transform of the propagator for the slow variables $j$ and $l$ that belong to the set $\{a\}$;

$$
\hat{g}_{j l}(\omega) \equiv \int_{0}^{\infty} \mathrm{d} t e^{\mathrm{i} \omega t} g_{j l}(t)=\mathrm{i}\left\langle 0\left|\alpha_{j} \frac{1}{\omega-\mathcal{H}} \tilde{\alpha}_{l}\right| 0\right\rangle
$$

We can manipulate it into

$$
\hat{g}_{j l}(\omega)=\mathrm{i}\left\langle 0_{a}\left|\alpha_{j} \frac{1}{\omega-\mathcal{H}_{a}(\omega)} \tilde{\alpha}_{l}\right| 0_{a}\right\rangle
$$

in order to have the frequency dependent effective "Hamiltonian" $\mathcal{H}_{a}(\omega)$, which acts on the variables $\{a\}$ with the interactions with the variables $\{b\}$ included and is given by

$$
\mathcal{H}_{a}(\omega)=\mathcal{H}_{0 a}+\left\langle 0_{b}\left|\mathcal{H}^{\prime}\right| 0_{b}\right\rangle+\left\langle 0_{b}\left|\mathcal{H}^{\prime}\left[\omega-\left(1-\mathcal{P}_{b}\right) \mathcal{H}\right]^{-1}\left(1-\mathcal{P}_{b}\right) \mathcal{H}^{\prime}\right| 0_{b}\right\rangle
$$

Now what we have to do for investigation of the slow modes is to know the scaling properties of the coefficients $\tilde{\omega}$ 's and $\tilde{\Omega}$ 's. We see that it is necessary and sufficient to have the scaling behaviors of the commutators of the reduced variables, i.e., $[\tilde{A}, \tilde{A}]$, which we will examine for the chiral phase transition in the next subsection. 


\subsection{Investigation of the chiral phase transition}

Firstly, we consider the scaling behavior of the amplitude of the slow variable $A_{j}$ itself. For the chiral phase transition, $A_{j}$ consists of $\left\{\phi_{\alpha \vec{k}}, \phi_{\alpha-\vec{k}}^{\dagger}, Q_{\vec{k}}, E_{\vec{k}}, P_{\vec{k}}^{i}\right\}$. These variables are the Fourier transforms of the corresponding density, which we will denote as $A_{j}(\vec{x})$. The $A_{j}(\vec{x})$ exhibits characteristic anomaly at the critical point and is scaled in terms of the correlation length $\xi$. To make it explicit, we write $A_{j}(\vec{x}, \xi, V)$ for $A_{j}(\vec{x})$ where we have also included the possible dependence on the volume $V$. Applying the block spin transformation with the block size $L$ on $A_{j}(\vec{x}, \xi, V)$, we have the scaled amplitude of the fluctuation as

$$
A_{j}(\vec{x}, \xi, V)=L^{-x_{j}} A_{j}\left(\vec{x} / L, \xi / L, V / L^{d}\right),
$$

where $x_{j}$ is the exponent to be determined. For the Fourier component which is defined by

$$
A(\vec{k})=\frac{1}{\sqrt{V}} \int \mathrm{d}^{d} x e^{-\mathrm{i} \vec{k} \cdot \vec{x}} A(\vec{x}),
$$

we then have

$$
A_{j}(\vec{k}, \xi, V)=L^{\frac{d}{2}-x_{j}} A_{j}\left(L \vec{k}, \xi / L, V / L^{d}\right) .
$$

The exponent $x_{j}$ can be determined by the (static) susceptibility $\chi_{j}$ :

$$
L^{d-2 x_{j}}\left\langle\left|A_{j}\left(\overrightarrow{0}, \xi / L, V / L^{d}\right)\right|^{2}\right\rangle=\left\langle\left|A_{j}(\overrightarrow{0}, \xi, V)\right|^{2}\right\rangle=\chi_{j, \vec{k}=\overrightarrow{0}} \sim \xi^{\frac{\gamma_{j}}{\nu}},
$$

where $\gamma_{j}$ and $\nu$ are the static critical exponents. When choosing $L=\xi$, we find immediately

$$
x_{j}=\frac{1}{2}\left(d-\frac{\gamma_{j}}{\nu}\right) .
$$

For the chiral phase transition, we have

$$
\gamma_{\phi}=\gamma, \quad \gamma_{Q}=0, \quad \gamma_{e}=\alpha, \quad \gamma_{p}=0
$$

leading to

$$
\begin{aligned}
& x_{\phi}=\frac{1}{2}\left(d-\frac{\gamma}{\nu}\right)=\frac{\beta}{\nu}, \\
& x_{Q}=\frac{d}{2}, \\
& x_{e}=\frac{1}{2}\left(d-\frac{\alpha}{\nu}\right)=d-\frac{1}{\nu}, \\
& x_{p}=\frac{d}{2},
\end{aligned}
$$

where we have used the scaling relations

$$
2-\alpha=d \nu, \quad \alpha+2 \beta+\gamma=2 .
$$


We note that the static critical exponents $\alpha, \beta, \gamma, \nu$ are the same as those of the ferro- and antiferromagnet because the chiral phase transition is in the same static universality class as these systems.

Thus the amplitudes of fluctuation $A_{j}$ have the characteristic scaling properties as follows;

$$
\begin{aligned}
\phi_{\alpha}(\vec{k}, \xi, V) & =\xi^{\frac{\gamma}{2 \nu}} \phi_{\alpha}\left(\xi \vec{k}, 1, V / \xi^{d}\right), \\
Q_{\alpha \beta}(\vec{k}, \xi, V) & =\xi^{0} Q_{\alpha \beta}\left(\xi \vec{k}, 1, V / \xi^{d}\right), \\
E(\vec{k}, \xi, V) & =\xi^{\frac{\alpha}{2 \nu}} E\left(\xi \vec{k}, 1, V / \xi^{d}\right), \\
P^{i}(\vec{k}, \xi, V) & =\xi^{0} P^{i}\left(\xi \vec{k}, 1, V / \xi^{d}\right) .
\end{aligned}
$$

We now discuss the scaling property of the commutators of $\tilde{A}_{j}$. If the exponent of $\left[A_{j}, A_{l}\right]$ is denoted as $x_{j l}$, namely $\left[A_{j}, A_{l}\right]$ scales as $\xi^{d / 2-x_{j l}}$ in the same sense as Eqs. (6.26), then we find that $\left[\tilde{A}_{j}, \tilde{A}_{l}\right]$ scales as $\xi^{x_{j}+x_{l}-d / 2-x_{j l}}$, that is,

$$
\left[\tilde{A}_{j}, \tilde{A}_{l}\right] \sim \xi^{x_{j}+x_{l}-\frac{d}{2}-x_{j l}}
$$

The computation of $x_{j l}$ for $\left\{\phi_{\alpha \vec{k}}, \phi_{\alpha-\vec{k}}^{\dagger}, Q_{\vec{k}}, E_{\vec{k}}, P_{\vec{k}}^{i}\right\}$ in the chiral phase transition is performed in Appendix C. Using those results, we find

$$
\begin{aligned}
& {\left[\tilde{\phi}, \tilde{\phi^{\dagger}}\right] \sim \xi^{-\frac{\gamma}{2 \nu}}, \quad[\tilde{\phi}, \tilde{\phi}]=\left[\tilde{\phi}^{\dagger}, \tilde{\phi^{\dagger}}\right]=0,} \\
& {[\tilde{\phi}, \tilde{Q}] \sim \xi^{-\frac{d}{2}}, \quad[\tilde{\phi}, \tilde{E}] \sim \xi^{-\frac{d}{2}-\frac{1}{\nu}}, \quad[\tilde{\phi}, \tilde{P}] \sim \xi^{-\frac{d}{2}-1},} \\
& {[\tilde{Q}, \tilde{Q}] \sim \xi^{-\frac{d}{2}}, \quad[\tilde{Q}, \tilde{E}] \sim \xi^{-\frac{d}{2}-\frac{1}{\nu}}, \quad[\tilde{Q}, \tilde{P}] \sim \xi^{-\frac{d}{2}-1},} \\
& {[\tilde{E}, \tilde{E}] \sim \xi^{\frac{d}{2}-1-\frac{2}{\nu}}=\xi^{-\frac{d}{2}-1-\frac{\alpha}{\nu}}, \quad[\tilde{E}, \tilde{P}] \sim \xi^{-\frac{d}{2}-1-\frac{1}{\nu}}=\xi^{-d-1-\frac{\alpha}{2 \nu}}} \\
& {[\tilde{P}, \tilde{P}] \sim \xi^{-\frac{d}{2}-1} .}
\end{aligned}
$$

We are now ready to derive the scaling behavior of the coefficients in the kinetic equation or in the "Hamiltonian." The nonvanishing frequencies of the propagating modes are those of the meson mode and the energy wave mode. The scaling properties for the reduced frequencies are

$$
\begin{gathered}
\tilde{\omega}_{\phi \vec{q}}=-k_{\mathrm{B}} T\left\langle\left[\tilde{\phi}_{\vec{q}}, \tilde{\phi}_{-\vec{q}}^{\dagger}\right]\right\rangle \sim \xi^{-\frac{\gamma}{2 \nu}}, \\
\tilde{\omega}_{e p^{\mathrm{L}} \vec{q}}=-k_{\mathrm{B}} T\left\langle\left[\tilde{E}_{\vec{q}}, \tilde{P}_{\vec{q}}^{\mathrm{L} \dagger}\right]\right\rangle \sim \xi^{-\frac{d}{2}-1-\frac{1}{\nu}} .
\end{gathered}
$$

For the scaling properties of the Onsager coefficients, we have

$$
L_{\phi}^{0} \sim \xi^{0}, \quad L_{Q}^{0} \sim \xi^{-2}, \quad \lambda^{0} \sim \eta^{0} \sim \zeta^{0} \sim \xi^{-2}
$$

because $\phi$ is not conserved while $Q, E$, and $P^{i}$ are conserved. We also note that

$$
\chi_{\phi} \sim \xi^{\frac{\gamma}{\nu}}, \quad \chi_{Q} \sim \chi_{p} \sim \xi^{0}, \quad \chi_{e} \sim \xi^{\frac{\alpha}{\nu}} .
$$


Thus we can find the scaling properties of the free "Hamiltonian" in the second quantized formalism. The free "Hamiltonian" $\mathcal{H}_{0}$ is separated into several parts:

$$
\mathcal{H}_{0}=\mathcal{H}_{0 \phi}^{N}+\mathcal{H}_{0 \phi}^{D}+\mathcal{H}_{0 Q}^{D}+\mathcal{H}_{0 e p^{\mathrm{L}}}^{N}+\mathcal{H}_{0 e}^{D}+\mathcal{H}_{0 p^{\mathrm{T}}}^{D}+\mathcal{H}_{0 p^{\mathrm{L}}}^{D}
$$

the scaling properties of which are found to be

$$
\begin{aligned}
& \mathcal{H}_{0 \phi}^{N} \sim \xi^{-\frac{\gamma}{2 \nu}}, \quad \mathcal{H}_{0 \phi}^{D} \sim \xi^{-\frac{\gamma}{\nu}}, \quad \mathcal{H}_{0 Q}^{D} \sim \xi^{-2}, \\
& \mathcal{H}_{0 e p^{\mathrm{L}}}^{N} \sim \xi^{-\frac{d}{2}-1-\frac{1}{\nu}}, \quad \mathcal{H}_{0 e}^{D} \sim \xi^{-2-\frac{\alpha}{\nu}} \\
& \mathcal{H}_{0 p^{\mathrm{T}}}^{D} \sim \xi^{-2}, \quad \mathcal{H}_{0 p^{\mathrm{L}}}^{D} \sim \xi^{-2-\frac{\alpha}{\nu}}+\xi^{-2} .
\end{aligned}
$$

We next turn to $\tilde{\Omega}_{j ; l m}$. This is contained in the interaction "Hamiltonian" $\mathcal{H}^{\prime}$ and determines its scaling property. We note that $\tilde{\Omega}_{j ; l m}$ involves the commutators of $\tilde{A}_{j}$. Hence it would be useful to divide the interaction "Hamiltonian" as

$$
\mathcal{H}^{\prime}=\mathcal{H}_{\phi}^{\prime}+\mathcal{H}_{Q}^{\prime}+\mathcal{H}_{e}^{\prime}+\mathcal{H}_{p}^{\prime}
$$

with

$$
\begin{aligned}
\mathcal{H}_{\phi}^{\prime} & =\mathcal{H}_{[\phi, \phi]}^{\prime}+\mathcal{H}_{[\phi, Q]}^{\prime}+\mathcal{H}_{[\phi, E]}^{\prime}+\mathcal{H}_{[\phi, P]}^{\prime}, \\
\mathcal{H}_{Q}^{\prime} & =\mathcal{H}_{[Q, \phi]}^{\prime}+\mathcal{H}_{[Q, Q]}^{\prime}+\mathcal{H}_{[Q, E]}^{\prime}+\mathcal{H}_{[Q, P]}^{\prime}, \\
\mathcal{H}_{e}^{\prime} & =\mathcal{H}_{[E, \phi]}^{\prime}+\mathcal{H}_{[E, Q]}^{\prime}+\mathcal{H}_{[E, E]}^{\prime}+\mathcal{H}_{[E, P]}^{\prime}, \\
\mathcal{H}_{p}^{\prime} & =\mathcal{H}_{[P, \phi]}^{\prime}+\mathcal{H}_{[P, Q]}^{\prime}+\mathcal{H}_{[P, E]}^{\prime}+\mathcal{H}_{[P, P]}^{\prime},
\end{aligned}
$$

where the scaling properties of each term are

$$
\begin{array}{rlrl}
\mathcal{H}_{[\phi, \phi]}^{\prime} & \sim \xi^{-\frac{\gamma}{2 \nu}}, & \mathcal{H}_{[\phi, Q]}^{\prime} & \sim \xi^{-\frac{d}{2}}, \quad \mathcal{H}_{[\phi, E]}^{\prime} \sim \xi^{-\frac{d}{2}-\frac{1}{\nu}}, \quad \mathcal{H}_{[\phi, P]}^{\prime} \sim \xi^{-\frac{d}{2}-1}, \\
\mathcal{H}_{[Q, Q]}^{\prime} & \sim \xi^{-\frac{d}{2}}, & \mathcal{H}_{[Q, E]}^{\prime} & \sim \xi^{-\frac{d}{2}-\frac{1}{\nu}}, \quad \mathcal{H}_{[Q, P]}^{\prime} \sim \xi^{-\frac{d}{2}-1}, \\
\mathcal{H}_{[E, E]}^{\prime} & \sim \xi^{\frac{d}{2}-1-\frac{2}{\nu}}, & \mathcal{H}_{[E, P]}^{\prime} \sim \xi^{-\frac{d}{2}-1-\frac{1}{\nu}}, \quad \mathcal{H}_{[P, P]}^{\prime} \sim \xi^{-\frac{d}{2}-1} .
\end{array}
$$

We are now in a position to discuss the dynamic behavior of each slow mode. We start with the dynamic behavior of the meson mode or the order parameter fluctuation. The information of the dynamic behavior is contained in the reduced propagator

$$
g_{\phi \vec{q} \alpha}(t)=\left\langle 0\left|\alpha_{\phi \vec{q} \alpha} e^{-i t \mathcal{H}} \tilde{\alpha}_{\phi \vec{q} \alpha}\right| 0\right\rangle,
$$

where $\alpha_{\phi \vec{q} \alpha}$ and $\tilde{\alpha}_{\phi \vec{q} \alpha}$ are the annihilation and creation operators for the mode $\phi_{\alpha \vec{q}}$. From Eqs. (6.13) and (6.14), we find that the Fourier transform of $g_{\phi \vec{q} \alpha}(t)$ 
is written as

$$
\hat{g}_{\phi \vec{q} \alpha}(\omega)=\mathrm{i}\left\langle 0_{\phi}\left|\alpha_{\phi \vec{q} \alpha} \frac{1}{\omega-\mathcal{H}_{\phi}(\omega)} \tilde{\alpha}_{\phi \vec{q} \alpha}\right| 0_{\phi}\right\rangle
$$

where

$$
\begin{aligned}
\mathcal{H}_{\phi}(\omega)= & \mathcal{H}_{0 \phi}^{N}+\mathcal{H}_{0 \phi}^{D}+\left\langle 0_{Q e p}\left|\mathcal{H}^{\prime}\right| 0_{Q e p}\right\rangle \\
& +\left\langle 0_{Q e p}\left|\mathcal{H}^{\prime} \frac{1}{\omega-\left(1-\mathcal{P}_{Q e p}\right) \mathcal{H}}\left(1-\mathcal{P}_{Q e p}\right) \mathcal{H}^{\prime}\right| 0_{Q e p}\right\rangle .
\end{aligned}
$$

The vacuum states $\left|0_{\phi}\right\rangle$ and $\left|0_{Q e p}\right\rangle$ are defined with respect to the modes $\{\phi\}$ and $\{Q, e, p\}$ respectively and $\mathcal{P}_{Q e p}$ is the projection operator onto $\left|0_{Q e p}\right\rangle$.

Here we need to use the general property of the interaction "Hamiltonian" [17]. The general argument tells us that the interaction "Hamiltonian" involving the commutator of a mode $j$ has at least one creation or annihilation operator of $j$. This means that

$$
\left\langle 0_{j}\left|\mathcal{H}_{[j, l]}^{\prime}\right| 0_{j}\right\rangle=0
$$

Moreover it is proved that the interaction "Hamiltonian" with $\Omega_{j ; l m}$, i.e., $\mathcal{H}_{j}^{\prime}$ has at least one creation operator as well as one annihilation operator of $j$. Thus both $\mathcal{H}_{j}^{\prime}\left|0_{j}\right\rangle$ and $\left\langle 0_{j}\right| \mathcal{H}_{j}^{\prime}$ vanish.

With these in mind, we find

$$
\left\langle 0_{Q e p}\left|\mathcal{H}^{\prime}\right| 0_{Q e p}\right\rangle=\left\langle 0_{Q e p}\left|\mathcal{H}_{[\phi, \phi]}^{\prime}\right| 0_{Q e p}\right\rangle \sim \xi^{-\frac{\gamma}{2 \nu}}
$$

As for the last term in Eq. (6.40), we note that at least one mode among $\{Q, e, p\}$ must be excited in intermediate states because of the presence of $1-\mathcal{P}_{Q e p}$. In order to make the last term as large as possible, it is desirable that the denominator is small. Because the "Hamiltonian" for the $\phi$ mode, $\mathcal{H}_{0 \phi}^{N} \sim \mathcal{H}_{[\phi, \phi]}^{\prime} \sim \xi^{-\gamma / 2 \nu}$, is larger than that of the other modes, the denominator would become larger in the absence of the $\phi$ mode in the intermediate state. However this is not allowed because

$$
\left\langle 0_{Q e p}\left|\mathcal{H}^{\prime}\right| 0_{\phi}\right\rangle=\left\langle 0_{\phi}\left|\mathcal{H}^{\prime}\right| 0_{Q e p}\right\rangle=0
$$

In the end, it turns out that the largest contribution is found in the presence of the $\phi$ mode in the intermediate state to be $\left(\mathcal{H}_{[\phi, \phi]}^{\prime}\right)^{2} /\left(\mathcal{H}_{0 \phi}^{N}+\mathcal{H}_{[\phi, \phi]}^{\prime}\right) \sim \xi^{-\gamma / 2 \nu}$. Thus the two interaction "Hamiltonian"s in the effective "Hamiltonian" for the $\phi$ mode are determined by the $\phi$ mode itself. This indicates that the $\phi$ mode almost decouples from the other modes.

We have clarified the scaling property of the effective "Hamiltonian" for the 
$\phi$ mode,

$$
\begin{aligned}
& \mathcal{H}_{\phi}= \mathcal{H}_{0 \phi}^{N}+\mathcal{H}_{0 \phi}^{D}+\left\langle 0_{Q e p}\left|\mathcal{H}^{\prime}\right| 0_{Q e p}\right\rangle \\
&+\left\langle 0_{Q e p}\left|\mathcal{H}^{\prime} \frac{1}{\omega-\left(1-\mathcal{P}_{Q e p}\right) \mathcal{H}}\left(1-\mathcal{P}_{Q e p}\right) \mathcal{H}^{\prime}\right| 0_{Q e p}\right\rangle \\
& \sim \xi^{-\frac{\gamma}{2 \nu}}+\xi^{-\frac{\gamma}{\nu}}+\xi^{-\frac{\gamma}{2 \nu}}+\xi^{-\frac{\gamma}{2 \nu}}
\end{aligned}
$$

The dynamics of the $\phi$ mode is dominated by $\mathcal{H}_{0 \phi}^{N}$ and the two interaction terms, all of which scale as $\xi^{-\gamma / 2 \nu}$. This means that $g_{\phi \vec{q} \alpha}(t)$ has the following form,

$$
g_{\phi \vec{q} \alpha}(t)=F\left(t \Gamma_{\phi \vec{q} \alpha}(\xi), q \xi\right)
$$

with the characteristic frequency of the form,

$$
\Gamma_{\phi \vec{q} \alpha}(\xi)=q^{\frac{\gamma}{2 \nu}} f(q \xi) .
$$

Thus we obtain the dynamic critical exponent $z$ for the $\phi$ mode

$$
z_{\phi}=\frac{\gamma}{2 \nu}=1-\frac{\eta}{2}
$$

When we use the values of the static critical exponents, $\eta=0.03$ [28], we find $z_{\phi} \cong 0.98$, which is contrasted with the value $z_{N}=d / 2=3 / 2$ of the staggered magnetization mode in the antiferromagnet.

Recently Boyanovsky and de Vega have calculated the dynamic critical exponent $z_{\phi}$ in a different approach [9]. They apply the renormalization group method directly to the microscopic theory, i.e., the field theory of the $\mathrm{O}(N)$ linear sigma model and obtain

$$
z=1+\epsilon \frac{N+2}{(N+8)^{2}}+\mathcal{O}\left(\epsilon^{2}\right)
$$

For $N=4$ and $\epsilon=1$, the above expression gives $z_{\phi} \cong 1.04$, which is compatible with our result rather than the value for the antiferromagnet.

We note that the fluctuation effect reduces the value to less than unity in our framework, while the opposite occurs in the calculation in Ref. [9]. The further close investigations of the kinetic equation, i.e., the application of the dynamic renormalization group method to the kinetic equation, may reveal the critical exponent larger than unity. Or it might be possible that the inclusion of higher order terms in the $\epsilon$ expansion and the proper resummation of those terms in the approach in Ref. [9] turn its tendency to the opposite. In any case, it needs more involved investigation to settle whether the dynamic critical exponent is larger or less than unity.

We note that if we employ the values in the large $N$ limit for $\eta$, that is, $\eta=0$, we have $z_{\phi}=1$. This is reasonable because the fluctuation is suppressed in the large $N$ limit. 
We find that the dissipation term $\mathcal{H}_{0 \phi}^{D}$ is smaller than the interaction terms. Thus the dissipation term is overwhelmed or renormalized by the interaction terms, which results in the same scaling pattern as the frequency term, that is, $\sim \xi^{-\gamma / 2 \nu}$. As a consequence, the Onsager coefficient diverges at the critical point as

$$
L_{\phi} \sim \xi^{\frac{\gamma}{2 \nu}} .
$$

What this means is that the width of the meson mode becomes narrower just in the same manner as that of the softening when we approach the critical point. This is not necessarily trivial. In fact, one of the motivations of the calculation in Ref. [9] is based on the suspicion that the meson mode may not be a good quasiparticle mode with a narrow width near the chiral phase transition. The calculation shows that the meson mode indeed becomes a good quasiparticle near the critical point, which agrees with our result.

As a final comment on the $\phi$ mode, we give the comparison with the antiferromagnet. In the antiferromagnet, the scaling property of the commutator of the staggered magnetization $\sigma$ is given by $[\tilde{\sigma}, \tilde{\sigma}] \sim \xi^{-d / 2}$ which leads to $\mathcal{H}^{\prime} \sim \xi^{-d / 2}$. The dissipation term $\mathcal{H}_{0 \sigma}^{D}$ scales as $\mathcal{H}_{0 \sigma}^{D} \sim \xi^{-\gamma / \nu}$ so that it is renormalized by $\mathcal{H}^{\prime}$ to scale as $\sim \xi^{-d / 2}$. This gives the critical exponent $z_{\sigma}=d / 2$, which is also predicted by the renormalization group method [20].

Next we consider the chiral charge $Q$. Using Eqs. (6.13) and (6.14), we have the propagator of the $Q$ mode as

$$
\hat{g}_{Q \vec{q} \alpha \beta}(\omega)=\mathrm{i}\left\langle 0_{Q}\left|\alpha_{Q \vec{q} \alpha \beta} \frac{1}{\omega-\mathcal{H}_{Q}(\omega)} \tilde{\alpha}_{Q \vec{q} \alpha \beta}\right| 0_{Q}\right\rangle
$$

where

$$
\begin{aligned}
\mathcal{H}_{Q}(\omega)= & \mathcal{H}_{0 Q}^{D}+\left\langle 0_{\phi e p}\left|\mathcal{H}^{\prime}\right| 0_{\phi e p}\right\rangle \\
& +\left\langle 0_{\phi e p}\left|\mathcal{H}^{\prime} \frac{1}{\omega-\left(1-\mathcal{P}_{\phi e p}\right) \mathcal{H}}\left(1-\mathcal{P}_{\phi e p}\right) \mathcal{H}^{\prime}\right| 0_{\phi e p}\right\rangle
\end{aligned}
$$

The second term reduces to

$$
\left\langle 0_{\phi e p}\left|\mathcal{H}_{[Q, Q]}^{\prime}\right| 0_{\phi e p}\right\rangle \sim \xi^{-\frac{d}{2}}
$$

The similar argument to the $\phi$ mode dynamics applies to the third term of Eq. (6.51). There arises the most important contribution from the situation where the intermediate state has the $Q$ mode as well as the $e$ or $p$ mode excitation but does not have the $\phi$ mode. Because the $Q$ mode "Hamiltonian," $\mathcal{H}_{[Q, Q]}^{\prime} \sim \xi^{-d / 2}$, is greater than those for the $e$ and $p$ modes, the $Q$ mode dominates in the intermediate state. Therefore the third term scales as $\left(\mathcal{H}_{[Q, Q]}^{\prime}\right)^{2} / \mathcal{H}_{[Q, Q]}^{\prime} \sim \xi^{-d / 2}$. As in the $\phi$ mode, the two interaction "Hamiltonian"s in the effective "Hamiltonian" $\mathcal{H}_{Q}$ are determined only by the $Q$ mode, 
which means that the $Q$ mode decouples from the other modes. Thus we find

$$
\begin{aligned}
\mathcal{H}_{Q} & =\mathcal{H}_{0 Q}^{D}+\left\langle 0_{\phi e p}\left|\mathcal{H}^{\prime}\right| 0_{\phi e p}\right\rangle+\left\langle 0_{\phi e p}\left|\mathcal{H}^{\prime} \frac{1}{\omega-\left(1-\mathcal{P}_{\phi e p}\right) \mathcal{H}}\left(1-\mathcal{P}_{\phi e p}\right) \mathcal{H}^{\prime}\right| 0_{\phi e p}\right\rangle \\
& \sim \xi^{-2}+\xi^{-\frac{d}{2}}+\xi^{-\frac{d}{2}}
\end{aligned}
$$

which gives us the dynamic critical exponent for the $Q$ mode,

$$
z_{Q}=\frac{d}{2}
$$

We see that the dissipation term receives the renormalization from the interaction terms and the Onsager coefficient diverges as

$$
L_{Q} \sim \xi^{2-\frac{d}{2}}
$$

The behavior of the $Q$ mode is quite the same as that of the magnetization $\mu$ in the antiferromagnet which is the counterpart of the $Q$ mode in the chiral phase transition. The magnetization is renormalized by the interaction terms to have the critical exponent $z_{\mu}=d / 2$, which is the same as that of the staggered magnetization. In this sense, the $Q$ mode may be regarded as a vestige of the antiferromagnet.

The third mode to be considered is the $p^{T}$ mode. The discussion goes in the similar way to before. The propagator for $p^{\mathrm{T}}$ is written as

$$
\hat{g}_{p^{\mathrm{T}} \vec{q} i}(\omega)=\mathrm{i}\left\langle 0_{p^{\mathrm{T}}}\left|\alpha_{p^{\mathrm{T}} \vec{q} i} \frac{1}{\omega-\mathcal{H}_{p^{\mathrm{T}}}(\omega)} \tilde{\alpha}_{p^{\mathrm{T}} \vec{q} i}\right| 0_{p^{\mathrm{T}}}\right\rangle,
$$

where

$$
\begin{aligned}
\mathcal{H}_{p^{\mathrm{T}}}(\omega)= & \mathcal{H}_{0 p^{\mathrm{T}}}^{D}+\left\langle 0_{\phi Q e p^{\mathrm{L}}}\left|\mathcal{H}^{\prime}\right| 0_{\phi Q e p^{\mathrm{L}}}\right\rangle \\
& +\left\langle 0_{\phi Q e p^{\mathrm{L}}}\left|\mathcal{H}^{\prime} \frac{1}{\omega-\left(1-\mathcal{P}_{\phi Q e p^{\mathrm{L}}}\right) \mathcal{H}}\left(1-\mathcal{P}_{\phi Q e p^{\mathrm{L}}}\right) \mathcal{H}^{\prime}\right| 0_{\phi Q e p^{\mathrm{L}}}\right\rangle .
\end{aligned}
$$

The scaling property of the second term in $\mathcal{H}_{p^{\mathrm{T}}}(\omega)$ is given by

$$
\left\langle 0_{\phi Q e p^{\mathrm{L}}}\left|\mathcal{H}^{\prime}\right| 0_{\phi Q e p^{\mathrm{L}}}\right\rangle=\left\langle 0_{\phi Q e p^{\mathrm{L}}}\left|\mathcal{H}_{\left[p^{\mathrm{T}}, p^{\mathrm{T}}\right]}^{\prime}\right| 0_{\phi Q e p^{\mathrm{L}}}\right\rangle \sim \xi^{-\frac{d}{2}-1} .
$$

For the third term, we have the most important contribution coming from the process with the $p^{\mathrm{T}}$ as well as the $e$ or $p^{\mathrm{L}}$ modes but without the $\phi$ and $Q$ modes in the intermediate state, which scales as $\sim \xi^{-d+\alpha / \nu}$. Thus we find

$$
\begin{aligned}
\mathcal{H}_{p^{\mathrm{T}}}= & \mathcal{H}_{0 p^{\mathrm{T}}}^{D}+\left\langle 0_{\phi Q e p^{\mathrm{L}}}\left|\mathcal{H}^{\prime}\right| 0_{\phi Q e p^{\mathrm{L}}}\right\rangle \\
& +\left\langle 0_{\phi Q e p^{\mathrm{L}}}\left|\mathcal{H}^{\prime} \frac{1}{\omega-\left(1-\mathcal{P}_{\phi Q e p^{\mathrm{L}}}\right) \mathcal{H}}\left(1-\mathcal{P}_{\phi Q e p^{\mathrm{L}}}\right) \mathcal{H}^{\prime}\right| 0_{\phi Q e p^{\mathrm{L}}}\right\rangle \\
\sim & \xi^{-2}+\xi^{-\frac{d}{2}-1}+\xi^{-d+\frac{\alpha}{\nu}}
\end{aligned}
$$


In the intermediate state in the third term, the $e$ and $p^{\mathrm{L}}$ modes couple to the $p^{T}$ mode. The most dominant term in the effective "Hamiltonian" is, however, not the interaction terms but the free term. Thus the $p^{T}$ mode again closes its dynamics within itself. The $p^{T}$ mode scales as $\sim \xi^{-2}$ without renormalization, leading to the critical exponent

$$
z_{p^{\mathrm{T}}}=2
$$

Since the renormalization is not effective, the Onsager coefficient of the transverse momentum, i.e., the shear viscosity, does not diverge at the critical point. This may be compared with the transverse velocity mode in the normal fluid in which the shear viscosity shows the logarithmic divergence. The logarithmic divergence is a consequence of renormalization from the third term with the $\xi^{-2}$ scaling. In the case of the normal fluid, there is a very slow mode, that is, the entropy mode which scales as $\xi^{-d}$. Because the intermediate state is dominated by this entropy mode, the denominator of the third term becomes very small, which results in the larger third term than ours.

Finally we consider the energy wave mode composed of the $e$ and $p^{\mathrm{L}}$ variables. The propagator is found to be

$$
\hat{g}_{e p^{\mathrm{L}} \vec{q} i}(\omega)=\mathrm{i}\left\langle 0_{e p^{\mathrm{L}}}\left|\alpha_{e p^{\mathrm{L}} \vec{q} i} \frac{1}{\omega-\mathcal{H}_{e p^{\mathrm{L}}}(\omega)} \tilde{\alpha}_{e p^{\mathrm{L}} \vec{q} i}\right| 0_{e p^{\mathrm{L}}}\right\rangle,
$$

where

$$
\begin{aligned}
\mathcal{H}_{e p^{\mathrm{L}}}(\omega)= & \mathcal{H}_{0 e p^{\mathrm{L}}}^{N}+\mathcal{H}_{0 e}^{D}+\mathcal{H}_{0 p^{\mathrm{L}}}^{D}+\left\langle 0_{\phi Q p^{\mathrm{T}}}\left|\mathcal{H}^{\prime}\right| 0_{\phi Q p^{\mathrm{T}}}\right\rangle \\
& +\left\langle 0_{\phi Q p^{\mathrm{T}}}\left|\mathcal{H}^{\prime} \frac{1}{\omega-\left(1-\mathcal{P}_{\phi Q p^{\mathrm{T}}}\right) \mathcal{H}}\left(1-\mathcal{P}_{\phi Q p^{\mathrm{T}}}\right) \mathcal{H}^{\prime}\right| 0_{\phi Q p^{\mathrm{T}}}\right\rangle .
\end{aligned}
$$

The first interaction term scales as

$$
\left\langle 0_{\phi Q p^{\mathrm{T}}}\left|\mathcal{H}^{\prime}\right| 0_{\phi Q p^{\mathrm{T}}}\right\rangle=\left\langle 0_{\phi Q p^{\mathrm{T}}}\left|\mathcal{H}_{[E, E]}^{\prime}+\mathcal{H}_{\left[E, P^{\mathrm{L}}\right]}^{\prime}+\mathcal{H}_{\left[P^{\mathrm{L}}, P^{\mathrm{L}}\right]}^{\prime}\right| 0_{\phi Q p^{\mathrm{T}}}\right\rangle \sim \xi^{\frac{d}{2}-1-\frac{2}{\nu}}
$$

The most important contribution of the second interaction term arises from the process in which the $e$ and $p^{\mathrm{L}}$ modes as well as the $p^{\mathrm{T}}$ mode appear but the $\phi$ and $Q$ modes are absent in the intermediate state, which gives the scaling behavior of the last term $\sim \xi^{d-(4-\alpha) / \nu}$. Thus we find

$$
\begin{aligned}
\mathcal{H}_{e p^{\mathrm{L}}}= & \mathcal{H}_{0 e p^{\mathrm{L}}}^{N}+\mathcal{H}_{0 e}^{D}+\mathcal{H}_{0 p^{\mathrm{L}}}^{D}+\left\langle 0_{\phi Q p^{\mathrm{T}}}\left|\mathcal{H}^{\prime}\right| 0_{\phi Q p^{\mathrm{T}}}\right\rangle \\
& +\left\langle 0_{\phi Q p^{\mathrm{T}}}\left|\mathcal{H}^{\prime} \frac{1}{\omega-\left(1-\mathcal{P}_{\phi Q p^{\mathrm{T}}}\right) \mathcal{H}}\left(1-\mathcal{P}_{\phi Q p^{\mathrm{T}}}\right) \mathcal{H}^{\prime}\right| 0_{\phi Q p^{\mathrm{T}}}\right\rangle \\
\sim & \xi^{-\frac{d}{2}-1-\frac{1}{\nu}}+\xi^{-2-\frac{\alpha}{\nu}}+\left(\xi^{-2-\frac{\alpha}{\nu}}+\xi^{-2}\right)+\xi^{\frac{d}{2}-1-\frac{2}{\nu}}+\xi^{d-\frac{4-\alpha}{\nu}}
\end{aligned}
$$

where the two terms in the parenthesis correspond to the terms associated with the thermal conductivity $\lambda^{0}$ and the bulk viscosity $\zeta^{0}$ in $\mathcal{H}_{0 p^{\mathrm{L}}}^{D}$. We see 
that the non-dissipation term is renormalized by the interaction terms, but the dissipation terms are the dominant terms and not renormalized, which give the critical exponent

$$
z_{e p^{\mathrm{L}}}=2+\frac{\alpha}{\nu} \cong 1.74
$$

with $\alpha=-0.19$ and $\nu=0.73$ substituted [28]. Thus the timescale of the energy wave mode is determined by the $e$ and $p^{\mathrm{L}}$ modes themselves and is not affected by the other modes. We note that the bulk viscosity does not diverge in contrast with the normal fluid. In the normal fluid, the commutator $\left[\tilde{p}, \tilde{u}^{\mathrm{L}}\right]$, that corresponds to $\left[\tilde{E}, \tilde{P}^{\mathrm{L}}\right]$ in our system, is very large. It enters into the non-dissipation term as well as into $\mathcal{H}^{\prime}$, the latter of which thus renormalizes the dissipation term. Because the slow entropy mode dominates the denominator, the second interaction term becomes even larger. Thus the bulk viscosity of the normal fluid shows the very strong divergence. In the chiral phase transition, $[\tilde{E}, \tilde{P} \mathrm{~L}]$ is somehow very small and the intermediate state in the second interaction term is not occupied by such a slow mode as the entropy mode. Thus the divergence of $\lambda$ and $\zeta$ does not occur.

From the above discussions on the four modes, we notice that the dynamic critical exponents, i.e., the time scales of each mode are all different and all the modes decouple from each other. As mentioned in Sec. 2.1, the original dynamic scaling hypothesis proposed in Ref. [19] claims that all the exponents are identical. In this sense, the "original" dynamic scaling hypothesis is violated in the present system.

Although the four modes decouple completely in our system, this should be regarded as an extreme case. In the antiferromagnet, the modes of the staggered magnetization and the magnetization couple to each other strongly. The critical exponents for the two modes are identical, though the energy mode has a different critical exponent. Thus the original dynamic scaling hypothesis holds partially in the antiferromagnet.

We note that in the chiral system, the largest critical exponent or the largest time scale is provided by the $p^{\mathrm{T}}$ mode. This means that the $p^{\mathrm{T}}$ mode is the slowest among the four modes. Thus it determines the relaxation time of the whole system. The other modes in themselves are certainly of interest. In particular, the softening and the narrowing of the meson mode have the many physical implications. But when we consider the relaxation of the system, it is dominated by the decay of the $p^{\mathrm{T}}$ mode, and the other modes are not relevant. 


\section{Summary}

We have discussed the dynamic aspect of the chiral phase transition. The classification method of the dynamic universality class had been established by Hohenberg and Halperin. The method is based on the prescription of how to collect the slow variables in the system. The prevailing prescription tells us to take the order parameter and conserved quantities of the system for the slow variables. If we follow the prescription, we are incorrectly led to identify the dynamic universality class of the chiral phase transition with that of the antiferromagnet and to find the dynamic critical exponent of the order parameter fluctuation to be $z=d / 2=1.5$, as Rajagopal and Wilczek had argued.

We have then turned into the consideration of the slow mode rather than the slow variable in order to find the crucial difference between the chiral phase transition and the antiferromagnet. While the order parameter fluctuation of the antiferromagnet in the disordered phase is a diffusive mode, the meson field which is the order parameter of the chiral phase transition gives apparently a propagating mode. We have stated that in order to describe the meson mode appropriately, we must include the canonical momentum conjugate to the meson field into the member of the slow variables. Since the slow modes, and accordingly the slow variables of the two systems, are different, it is impossible to say that the dynamic universality class of the two systems are identical. What this means is the breakdown of the prescription for choosing the slow variables just by gathering the order parameter and the conserved quantities, because the canonical momentum for the meson field is neither the order parameter nor the conserved quantity. In the chiral phase transition, the order parameter and the conserved quantity does not furnish us with the full slow variables required to describe the slow mode properly. We have dangerous potentiality that the dynamic universality class given by Hohenberg and Halperin's classification method is divided into finer classes, if we consider the slow modes and their appropriate slow variables in that dynamic universality class.

The necessity of the canonical momentum for the slow variable is itself nothing strange. When we return to the microscopic eqution of motion, i.e., the Heisenberg equation for the meson field, we can immediately realize that the canonical momentum in addition to the meson field is necessary to describe the meson dynamics. Moreover we have mentioned that the canonical momentum as a slow variable is not restricted to the chiral phase transition. In the suprefluid and the ferromagnet, the canonical momentum plays the role of the slow variable in order to describe the second sound and the spin wave respectively. In the two systems, the canonical momentum is just an order parameter or a conserved quantity, and can become the slow variable just by 
the old prescription. In the chiral phase transition, on the other, the canonical momentum is not the order parameter or the conserved quantity. This is just the only new feature of the chiral phase transition.

The above observation has inspired the necessity of reanalysis of the chiral phase transition. We have employed the mode coupling theory for that purpose.

Firstly, we have applied the theory to the $\mathrm{O}(2)$ linear sigma model and found that the meson mode appears desirably from the meson field and the canonical momentum. We have also clarified the other slow modes than the meson mode: The chiral charge gives a diffusive mode in the disordered phase. In the ordered phase, it couples with the pion field, which results in lifting the degeneracy of the pion mode with the sigma mode. The energy and the longitudinal momentum are combined to give the energy wave mode, which is a propagating mode and a correspondent to a sound wave in a fluid. The transverse momenta give diffusive modes.

We have then examined the $\mathrm{O}(4)$ linear sigma model in the mode coupling theory in order to derive the kinetic equation and calculate the dynamic critical exponents in the disordered phase. We have found the exponent for the meson mode to be $z_{\phi}=1-\eta / 2 \cong 0.98$, which is to be compared with the value of $d / 2$ obtained assuming that the chiral phase transition belongs to the dynamic universality class of the antiferromagnet. The different dynamic critical exponents show explicitly the different dynamic behavior. We have also calculated the dynamic critical exponents of the modes other than the meson mode. The results are given in Eqs. (6.54), (6.60) and (6.65). We have noted that the largest exponent is given by the transverse momentum fluctuation, which determines the relaxation time of the whole system in the chiral phase transition.

We have succeeded in describing the meson mode by including the canonical momentum into the member of the slow variables. We were able to find, even accidentally, the needed slow variable, that is, the canonical momentum by returning to the microscopic Heisenberg equation. However, we must admit that the argument does not go beyond a heuristic one. We do not have any definite prescription to determine the slow variables. It will be our future problem to find out the way how to determine the slow variables uniquely, if it exists. Once it is found, it means that we have obtained a new classification method for the dynamic universality class, which completes that of Hohenberg and Halperin.

We note that a "particle mode" like the meson mode is inherent in the relativistic system because it accompanies the anti-particle mode as a partner. As we have seen, for the description of the "particle mode," we need the canoni- 
cal momentum that is neither the order parameter nor the conserved quantity. Thus the relativistic system that involves the "particle mode" as a slow mode should be a quite novel critical point that is not classified into any dynamic universality classes of the non-relativistic systems considered by Hohenberg and Halperin in Ref. [14]. One of such a system would be the critical end point (CEP) in the QCD phase diagram [29,30,31,32,33,34,35]. Although the dynamic universality class of the CEP is discussed in Ref. [35], the argument is not sufficient in respect that only the order parameter and the conserved quantities are compared and the slow modes are not taken into account explicitly. Other new and intriguing systems would be the tricritical point, the critical point associated with the color-superconductivity, the confinement transition, the electro-weak transition and so on. The analysis of those systems including CEP should also be what we should do in the future.

\section{Acknowledgments}

Two of the authors (K. O and K. O) are grateful to all the members of the nuclear theory group at Komaba in the university of Tokyo for useful discussions. K. Ohnishi thanks Prof. T. Hatsuda for precious comments and discussions. He also thanks Dr. M. Ohtani for fruitfull discussions. He had an opportunity

to have stimulating discussions with Prof. T. Kunihiro during stay at YITP as its visitor program. He would like to express his gratitude to Pror. Kunihiro. K. F is supported by Japan Society for the Promotion of Science for Young Scientists.

\section{A Commutation relations of the slow variables}

In this appendix, we list up the commutation relations among the slow variables. 


$$
\begin{aligned}
& {\left[\phi_{\alpha \vec{q}},\left(\phi_{\alpha^{\prime} \vec{q}^{\prime}}\right)^{\dagger}\right]=\frac{1}{2 \omega_{\vec{q}}} \delta_{\alpha \alpha^{\prime}} \delta_{\vec{q} \vec{q}^{\prime}}} \\
& {\left[\phi_{\alpha \vec{q}},\left(\phi_{\alpha^{\prime}-\vec{q}^{\prime}}^{\dagger}\right)^{\dagger}\right]=0} \\
& {\left[\phi_{\alpha \vec{q}},\left(Q_{\alpha^{\prime} \beta^{\prime} \vec{q}^{\prime}}\right)^{\dagger}\right]} \\
& =\frac{-\mathrm{i}}{2 \sqrt{V} \sqrt{2 \omega_{\vec{q}}}} \epsilon_{\alpha \alpha^{\prime} \beta^{\prime} \gamma}\left\{\sqrt{\frac{\omega_{\vec{q}}}{\omega_{\vec{q}-\vec{q}^{\prime}}}}\left(a_{\gamma \vec{q}-\vec{q}^{\prime}}+a_{\gamma \vec{q}^{\prime}-\vec{q}}^{\dagger}\right)+\sqrt{\frac{\omega_{\vec{q}-\vec{q}^{\prime}}}{\omega_{\vec{q}}}}\left(a_{\gamma \vec{q}-\vec{q}^{\prime}}-a_{\gamma \vec{q}^{\prime}-\vec{q}}^{\dagger}\right)\right\} \\
& {\left[\phi_{\alpha \vec{q}},\left(E_{\vec{q}^{\prime}}\right)^{\dagger}\right]=\frac{1}{2 \sqrt{V} \sqrt{2 \omega_{\vec{q}}}} \sqrt{\omega_{\vec{q}} \omega_{\vec{q}-\vec{q}^{\prime}}}\left(a_{\alpha \vec{q}-\vec{q}^{\prime}}-a_{\alpha \vec{q}^{\prime}-\vec{q}}^{\dagger}\right)} \\
& +\frac{1}{2 \sqrt{V} \sqrt{2 \omega_{\vec{q}}}} \frac{1}{\sqrt{\omega_{\vec{q}} \omega_{\vec{q}-\vec{q}^{\prime}}}}\left[\vec{q} \cdot\left(\vec{q}-\vec{q}^{\prime}\right)+\mu^{2}\right]\left(a_{\alpha \vec{q}-\vec{q}^{\prime}}+a_{\alpha \vec{q}^{\prime}-\vec{q}}^{\dagger}\right) \\
& +\frac{\lambda}{V^{\frac{3}{2}} \sqrt{2 \omega_{\vec{q}}}} \sum_{\vec{k}_{1} \cdots \vec{k}_{4}} \frac{1}{4} \frac{1}{\sqrt{\omega_{\vec{k}_{1}} \cdots \omega_{\vec{k}_{4}}}} \delta_{-\vec{q}^{\prime}, \vec{k}_{1}+\cdots+\vec{k}_{4}} \delta_{-\vec{q}, \vec{k}_{1}} \\
& \times\left(a_{\alpha \vec{k}_{2}}+a_{\alpha-\vec{k}_{2}}^{\dagger}\right)\left(a_{\beta \vec{k}_{3}}+a_{\beta-\vec{k}_{3}}^{\dagger}\right)\left(a_{\beta \vec{k}_{4}}+a_{\beta-\vec{k}_{4}}^{\dagger}\right) \\
& {\left[\phi_{\alpha \vec{q}},\left(P_{\vec{q}^{\prime}}^{i}\right)^{\dagger}\right]} \\
& =\frac{1}{2 \sqrt{V} \sqrt{2 \omega_{\vec{q}}}}\left\{\sqrt{\frac{\omega_{\vec{q}}}{\omega_{\vec{q}-\vec{q}^{\prime}}}}\left(q-q^{\prime}\right)^{i}\left(a_{\alpha \vec{q}-\vec{q}^{\prime}}+a_{\alpha \vec{q}^{\prime}-\vec{q}}^{\dagger}\right)+\sqrt{\frac{\omega_{\vec{q}-\vec{q}^{\prime}}}{\omega_{\vec{q}}}} q^{i}\left(a_{\alpha \vec{q}-\vec{q}^{\prime}}-a_{\alpha \vec{q}^{\prime}-\vec{q}}^{\dagger}\right)\right\} \\
& {\left[\phi_{\alpha-\vec{q}}^{\dagger},\left(\phi_{\alpha^{\prime}-\vec{q}^{\prime}}^{\dagger}\right)^{\dagger}\right]=-\frac{1}{2 \omega_{\vec{q}}} \delta_{\alpha \alpha^{\prime}} \delta_{\vec{q} \vec{q}^{\prime}}} \\
& {\left[\phi_{\alpha-\vec{q}}^{\dagger},\left(Q_{\alpha^{\prime} \beta^{\prime} \vec{q}^{\prime}}\right)^{\dagger}\right]=-\left[\phi_{\alpha-\vec{q}}, Q_{\alpha^{\prime} \beta^{\prime} \vec{q}^{\prime}}\right]^{\dagger}} \\
& =\frac{-\mathrm{i}}{2 \sqrt{V} \sqrt{2 \omega_{\vec{q}}}} \epsilon_{\alpha \alpha^{\prime} \beta^{\prime} \gamma}\left\{\sqrt{\frac{\omega_{\vec{q}}}{\omega_{\vec{q}-\vec{q}^{\prime}}}}\left(a_{\gamma \vec{q}-\vec{q}^{\prime}}+a_{\gamma \vec{q}^{\prime}-\vec{q}}^{\dagger}\right)-\sqrt{\frac{\omega_{\vec{q}-\vec{q}^{\prime}}}{\omega_{\vec{q}}}}\left(a_{\gamma \vec{q}-\vec{q}^{\prime}}-a_{\gamma \vec{q}^{\prime}-\vec{q}}^{\dagger}\right)\right\}
\end{aligned}
$$




$$
\begin{aligned}
{\left[\phi_{\alpha-\vec{q}}^{\dagger},\left(E_{\vec{q}^{\prime}}\right)^{\dagger}\right]=} & -\left[\phi_{\alpha-\vec{q}}, E_{\vec{q}^{\prime}}\right]^{\dagger} \\
= & \frac{1}{2 \sqrt{V} \sqrt{2 \omega_{\vec{q}}}} \sqrt{\omega_{\vec{q}} \omega_{\vec{q}-\vec{q}^{\prime}}}\left(a_{\alpha \vec{q}-\vec{q}^{\prime}}-a_{\alpha \vec{q}^{\prime}-\vec{q}}^{\dagger}\right) \\
& -\frac{1}{2 \sqrt{V} \sqrt{2 \omega_{\vec{q}}}} \frac{1}{\sqrt{\omega_{\vec{q}} \omega_{\vec{q}-\vec{q}^{\prime}}}}\left[\vec{q} \cdot\left(\vec{q}-\vec{q}^{\prime}\right)+\mu^{2}\right]\left(a_{\alpha \vec{q}-\vec{q}^{\prime}}+a_{\alpha \vec{q}^{\prime}-\vec{q}}^{\dagger}\right) \\
& -\frac{\lambda}{V^{\frac{3}{2}} \sqrt{2 \omega_{\vec{q}}}} \sum_{\vec{k}_{1} \cdots \vec{k}_{4}} \frac{1}{4} \frac{1}{\sqrt{\omega_{\vec{k}_{1}} \cdots \omega_{\overrightarrow{k_{4}}}}} \delta_{-\vec{q}^{\prime}, \vec{k}_{1}+\cdots+\vec{k}_{4}} \delta_{-\vec{q}, \vec{k}_{1}} \\
& \times\left(a_{\alpha \vec{k}_{2}}+a_{\alpha-\vec{k}_{2}}^{\dagger}\right)\left(a_{\beta \vec{k}_{3}}+a_{\beta-\vec{k}_{3}}^{\dagger}\right)\left(a_{\beta \vec{k}_{4}}+a_{\beta-\vec{k}_{4}}^{\dagger}\right)
\end{aligned}
$$

$$
\begin{aligned}
& {\left[\phi_{\alpha-\vec{q}}^{\dagger},\left(P_{\vec{q}^{\prime}}^{i}\right)^{\dagger}\right]=-\left[\phi_{\alpha-\vec{q}}, P_{\vec{q}^{\prime}}^{i}\right]^{\dagger}} \\
& \quad=\frac{1}{2 \sqrt{V} \sqrt{2 \omega_{\vec{q}}}}\left\{\sqrt{\frac{\omega_{\vec{q}}}{\omega_{\vec{q}-\vec{q}^{\prime}}}}\left(q-q^{\prime}\right)^{i}\left(a_{\alpha \vec{q}-\vec{q}^{\prime}}+a_{\alpha \vec{q}^{\prime}-\vec{q}}^{\dagger}\right)-\sqrt{\frac{\omega_{\vec{q}-\vec{q}^{\prime}}}{\omega_{\vec{q}}}} q^{i}\left(a_{\alpha \vec{q}-\vec{q}^{\prime}}-a_{\alpha \vec{q}^{\prime}-\vec{q}}^{\dagger}\right)\right\}
\end{aligned}
$$

$$
\begin{aligned}
& {\left[Q_{a \vec{q}}^{V},\left(Q_{b \vec{q}^{\prime}}^{V}\right)^{\dagger}\right]=\frac{\mathrm{i}}{\sqrt{V}} \epsilon_{a b c} Q_{c \vec{q}-\vec{q}^{\prime}}^{V}} \\
& {\left[Q_{a \vec{q}}^{V},\left(Q_{b \vec{q}^{\prime}}^{A}\right)^{\dagger}\right]=\frac{\mathrm{i}}{\sqrt{V}} \epsilon_{a b c} Q_{c \vec{q}-\vec{q}^{\prime}}^{A}}
\end{aligned}
$$

$$
\left[Q_{a \vec{q}}^{A},\left(Q_{b \vec{q}^{\prime}}^{A}\right)^{\dagger}\right]=\frac{\mathrm{i}}{\sqrt{V}} \epsilon_{a b c} Q_{c \vec{q}-\vec{q}^{\prime}}^{V}
$$

$$
\left[Q_{\alpha \beta \vec{q}},\left(E_{\vec{q}^{\prime}}\right)^{\dagger}\right]=-\mathrm{i} \epsilon_{\alpha \beta \gamma \delta} \frac{1}{V} \sum_{\vec{k}} \frac{1}{2} \frac{1}{\sqrt{\omega_{\vec{k}} \omega_{\vec{q}-\vec{q}^{\prime}-\vec{k}}}}
$$

$$
\times(\vec{q}-\vec{k}) \cdot\left(\vec{q}-\vec{q}^{\prime}-\vec{k}\right)\left(a_{\gamma \vec{k}}+a_{\gamma-\vec{k}}^{\dagger}\right)\left(a_{\delta \vec{q}-\vec{q}^{\prime} \vec{k}}+a_{\delta \vec{k}+\vec{q}^{\prime}-\vec{q}}^{\dagger}\right)
$$

$\left[Q_{\alpha \beta \vec{q}},\left(P_{\vec{q}^{\prime}}^{i}\right)^{\dagger}\right]=\frac{1}{\sqrt{V}} q^{i} Q_{\alpha \beta \vec{q}-\vec{q}^{\prime}}$

$$
\left[E_{\vec{q}},\left(E_{\vec{q}^{\prime}}\right)^{\dagger}\right]=\frac{1}{\sqrt{V}}\left(q+q^{\prime}\right)^{i} P_{\vec{q}-\vec{q}^{\prime}}^{i}
$$

$$
\begin{aligned}
{\left[E_{\vec{q}},\left(P_{\vec{q}^{\prime}}^{i}\right)^{\dagger}\right]=} & -\frac{1}{2}\left(q+q^{\prime}\right)^{i} \frac{1}{V} \sum_{\vec{k}} \frac{1}{2} \sqrt{\omega_{\vec{k}} \omega_{\vec{q}-\vec{q}^{\prime}-\vec{k}}}\left(a_{\alpha \vec{k}}-a_{\alpha-\vec{k}}^{\dagger}\right)\left(a_{\alpha \vec{q}-\vec{q}^{\vec{q}}-\vec{k}}-a_{\alpha \vec{k}+\vec{q}^{\prime}-\vec{q}}^{\dagger}\right) \\
+ & \frac{1}{V} \sum_{\vec{k}} \frac{1}{2} \frac{1}{\sqrt{\omega_{\vec{k}} \omega_{\vec{q}-\vec{q}^{\prime}-\vec{k}}}}\left[-\vec{q} \cdot \vec{k}+\vec{k}^{2}+\mu^{2}\right]\left(q-q^{\prime}-k\right)^{i} \\
& \times\left(a_{\alpha \vec{k}}+a_{\alpha-\vec{k}}^{\dagger}\right)\left(a_{\alpha \vec{q}-\vec{q}^{\prime}-\vec{k}}+a_{\alpha \vec{k}+\vec{q}^{\prime}-\vec{q}}^{\dagger}\right) \\
+ & \frac{\lambda}{V^{2}} \sum_{\vec{k}_{1} \cdots \vec{k}_{4}} \frac{1}{4} \frac{1}{\sqrt{\omega_{\vec{k}_{1}} \cdots \omega_{\vec{k}}}} k_{4}^{i} \delta_{\vec{q}-\vec{q}^{\prime}, \vec{k}_{1}+\cdots+\vec{k}_{4}} \\
& \quad \times\left(a_{\beta \vec{k}_{1}}+a_{\beta-\vec{k}_{1}}^{\dagger}\right)\left(a_{\beta \vec{k}_{2}}+a_{\beta-\vec{k}_{2}}^{\dagger}\right)\left(a_{\alpha \vec{k}_{3}}+a_{\alpha-\vec{k}_{3}}^{\dagger}\right)\left(a_{\alpha \vec{k}_{4}}+a_{\alpha-\vec{k}_{4}}^{\dagger}\right)
\end{aligned}
$$

$$
\left[P_{\vec{q}}^{i},\left(P_{\vec{q}^{\prime}}^{j}\right)^{\dagger}\right]=\frac{1}{V}\left(q^{j} P_{\vec{q}-\vec{q}^{\prime}}^{i}+q^{\prime i} P_{\vec{q}-\vec{q}^{\prime}}^{j}\right)
$$




\section{B Dissipation terms in the relativistic hydrodynamics}

The dissipation terms for $E_{\vec{q}}$ and $P_{\vec{q}}^{i}$ are read from the relativistic hydrodynamic equation [36]. We will perform it in this appendix. The energymomentum tensor for the imperfect fluid is given by

$$
T^{\alpha \beta}=T_{0}^{\alpha \beta}+\Delta T^{\alpha \beta}
$$

where

$$
\begin{aligned}
T_{0}^{\alpha \beta} & =p \eta^{\alpha \beta}+(p+\rho) U^{\alpha} U^{\beta} \\
\Delta T^{\alpha \beta} & =-\eta H^{\alpha \gamma} H^{\beta \delta} W_{\gamma \delta}-\lambda\left(H^{\alpha \gamma} U^{\beta}+H^{\beta \gamma} U^{\alpha}\right) Q_{\gamma}-\zeta H^{\alpha \beta} \frac{\partial U^{\gamma}}{\partial x^{\gamma}} .
\end{aligned}
$$

The $T_{0}^{\alpha \beta}$ is for the perfect fluid and $\Delta T^{\alpha \beta}$ takes care of the dissipation effects. The $p$ and $\rho$ are the pressure and the proper energy density. The coefficients $\lambda, \eta, \zeta$ are the heat conductivity, the shear viscosity and the bulk viscosity, respectively. The metric tensor $\eta^{\alpha \beta}$ are taken to be $\eta^{\alpha \beta}=\operatorname{diag}(-1,1,1,1)$. The four velocity $U^{\alpha}=\left(1 / \sqrt{1-v^{2}}, \vec{v} / \sqrt{1-v^{2}}\right)$ has the property $U^{\alpha} U_{\alpha}=-1$ in this metric. The $W_{\alpha \beta}, Q_{\alpha}, H_{\alpha \beta}$ are defined by

$$
\begin{aligned}
W_{\alpha \beta} & =\frac{\partial U_{\alpha}}{\partial x^{\beta}}+\frac{\partial U_{\beta}}{\partial x^{\alpha}}-\frac{2}{3} \eta_{\alpha \beta} \frac{\partial U^{\gamma}}{\partial x^{\gamma}} \\
Q_{\alpha} & =\frac{\partial T}{\partial x^{\alpha}}+T \frac{\partial U_{\alpha}}{\partial x^{\beta}} U^{\beta} \\
H_{\alpha \beta} & =\eta_{\alpha \beta}+U_{\alpha} U_{\beta} .
\end{aligned}
$$

We define the energy density and the momentum density as

$$
\begin{aligned}
E(\vec{x}, t) & =T_{0}^{00}=\frac{\rho+p v^{2}}{1-v^{2}} \\
P^{i}(\vec{x}, t) & =T_{0}^{i 0}=(p+\rho) \frac{v^{i}}{1-v^{2}}
\end{aligned}
$$

We can derive the relation between $U^{\mu}$ and $P^{i}$. ¿From Eq. (B.8), we see

$$
P^{i}=(p+\rho) U^{0} U^{i}
$$

Moreover using $U^{\alpha} U_{\alpha}=-1$, we find

$$
U^{0}=\sqrt{1+\frac{1}{(p+\rho)^{2}} P^{j} P_{j}} \cong 1+\frac{1}{2(p+\rho)^{2}} P^{j} P_{j}
$$

in the approximation of the small momentum fluctuation $P^{i} \ll 1$. 
Now consider the hydrodynamic equation. It is given by the energy-momentum conservation,

$$
0=\frac{\partial T^{\alpha \beta}}{\partial x^{\beta}}=\frac{\partial T_{0}^{\alpha \beta}}{\partial x^{\beta}}+\frac{\partial \Delta T^{\alpha \beta}}{\partial x^{\beta}},
$$

from which we find

$$
\begin{aligned}
\frac{\partial}{\partial t} E & =-\frac{\partial P^{j}}{\partial x^{j}}-\frac{\partial \Delta T^{0 \beta}}{\partial x^{\beta}} \\
\frac{\partial}{\partial t} P^{i} & =-\frac{\partial T_{0}^{i j}}{\partial x^{j}}-\frac{\partial \Delta T^{i \beta}}{\partial x^{\beta}} .
\end{aligned}
$$

The second terms in the right hand sides are the dissipation terms, which we will compute in the following. The $\Delta T^{\alpha \beta}$ are calculated to be

$$
\begin{aligned}
\Delta T^{\alpha \beta}= & -\lambda\left[2 U^{\alpha} U^{\beta} U^{\gamma} \frac{\partial T}{\partial x^{\gamma}}+U^{\alpha} \frac{\partial T}{\partial x_{\beta}}+U^{\beta} \frac{\partial T}{\partial x_{\alpha}}+T U^{\gamma} \frac{\partial\left(U^{\alpha} U^{\beta}\right)}{\partial x^{\gamma}}\right] \\
& -\eta\left[\frac{\partial U^{\alpha}}{\partial x_{\beta}}+\frac{\partial U^{\beta}}{\partial x_{\alpha}}-\frac{2}{3}\left(\eta^{\alpha \beta}+U^{\alpha} U^{\beta}\right) \frac{\partial U^{\gamma}}{\partial x^{\gamma}}+U^{\gamma} \frac{\partial\left(U^{\alpha} U^{\beta}\right)}{\partial x^{\gamma}}\right] \\
& -\zeta\left(\eta^{\alpha \beta}+U^{\alpha} U^{\beta}\right) \frac{\partial U^{\gamma}}{\partial x^{\gamma}} .
\end{aligned}
$$

Thus the dissipation term for the energy density is

$$
\begin{aligned}
-\frac{\partial \Delta T^{0 \beta}}{\partial x^{\beta}}=\frac{\partial}{\partial x^{0}} & \lambda\left(2 U^{0} U^{0} U^{\gamma} \frac{\partial T}{\partial x^{\gamma}}+2 U^{0} \frac{\partial T}{\partial x_{0}}+T U^{\gamma} \frac{\partial\left(U^{0} U^{0}\right)}{\partial x^{\gamma}}\right) \\
+ & \eta\left(2 \frac{\partial U^{0}}{\partial x_{0}}-\frac{2}{3}\left(-1+U^{0} U^{0}\right) \frac{\partial U^{\gamma}}{\partial x^{\gamma}}+U^{\gamma} \frac{\partial\left(U^{0} U^{0}\right)}{\partial x^{\gamma}}\right) \\
+ & \left.\zeta\left(-1+U^{0} U^{0}\right) \frac{\partial U^{\gamma}}{\partial x^{\gamma}}\right] \\
+\frac{\partial}{\partial x^{j}} & {\left[\lambda\left(2 U^{0} U^{j} U^{\gamma} \frac{\partial T}{\partial x^{\gamma}}+U^{0} \frac{\partial T}{\partial x_{j}}+U^{j} \frac{\partial T}{\partial x_{0}}+T U^{\gamma} \frac{\partial\left(U^{0} U^{j}\right)}{\partial x^{\gamma}}\right)\right.} \\
+ & \eta\left(\frac{\partial U^{0}}{\partial x_{j}}+\frac{\partial U^{j}}{\partial x_{0}}-\frac{2}{3} U^{0} U^{j} \frac{\partial U^{\gamma}}{\partial x^{\gamma}}+U^{\gamma} \frac{\partial\left(U^{0} U^{j}\right)}{\partial x^{\gamma}}\right) \\
+ & \left.\zeta U^{0} U^{j} \frac{\partial U^{\gamma}}{\partial x^{\gamma}}\right]
\end{aligned}
$$

Here the temperature $T$ is related to $E$ through the thermodynamic relation

$$
\delta T=\frac{1}{C} \delta E
$$

with $C$ being the specific heat. The $U^{0}$ and $U^{j}$ are translated to $P^{j}$ by Eqs. (B.9) and (B.10). Moreover following the non-relativistic case, we take only the terms up to the third order of the assumed small quantities, that is, the frequency and wavenumber as well as the fluctuations $E$ and $P^{i}$. Since each 
term in Eq. (B.15) involves two time and space derivatives or the frequency and wavenumber, we have only to take the first order of $E$ and $P^{i}$. Thus we find

$$
\begin{aligned}
-\frac{\partial \Delta T^{\alpha \beta}}{\partial x^{\beta}} & =\lambda \frac{\partial}{\partial x^{j}}\left[\frac{1}{C} \frac{\partial E}{\partial x_{j}}+T \frac{\partial}{\partial t}\left(\frac{1}{\rho+p} P^{j}\right)\right] \\
& \cong \lambda \frac{\partial}{\partial x^{j}}\left(\frac{1}{C} \frac{\partial E}{\partial x_{j}}+T_{0} \frac{1}{\rho_{0}+p_{0}} \frac{\partial P^{j}}{\partial t}\right)
\end{aligned}
$$

where in the last step, we replaced $T, \rho, p$ with their equilibrium values $T_{0}$, $\rho_{0}, p_{0}$.

Similarly, the dissipation term for the momentum density becomes

$$
\begin{aligned}
-\frac{\partial \Delta T^{i \beta}}{\partial x^{\beta}}= & \lambda \frac{\partial}{\partial t}\left[\frac{1}{C} \frac{\partial E}{\partial x_{i}}+T_{0} \frac{1}{\rho_{0}+p_{0}} \frac{\partial P^{i}}{\partial t}\right] \\
& +\eta \frac{1}{\rho_{0}+p_{0}} \frac{\partial}{\partial x^{j}}\left(\frac{\partial P^{i}}{\partial x_{j}}+\frac{\partial P^{j}}{\partial x_{i}}-\frac{2}{3} \eta^{i j} \frac{\partial P^{l}}{\partial x^{l}}\right) \\
& +\zeta \frac{1}{\rho_{0}+p_{0}} \frac{\partial}{\partial x_{i}} \frac{\partial P^{l}}{\partial x^{l}} .
\end{aligned}
$$

The hydrodynamic equation with the dissipation term is thus

$$
\begin{aligned}
\frac{\partial E}{\partial t}= & -\frac{\partial P^{j}}{\partial x^{j}}+\lambda \frac{\partial}{\partial x^{j}}\left(\frac{1}{C} \frac{\partial E}{\partial x_{j}}+\frac{T_{0}}{\rho_{0}+p_{0}} \frac{\partial P^{j}}{\partial t}\right) \\
\frac{\partial P^{i}}{\partial t}= & -\frac{\partial T_{0}^{i j}}{\partial x^{j}}+\lambda \frac{\partial}{\partial t}\left(\frac{1}{C} \frac{\partial E}{\partial x_{i}}+\frac{T_{0}}{\rho_{0}+p_{0}} \frac{\partial P^{i}}{\partial t}\right) \\
& +\eta \frac{1}{\rho_{0}+p_{0}} \frac{\partial}{\partial x^{j}}\left(\frac{\partial P^{i}}{\partial x_{j}}+\frac{\partial P^{j}}{\partial x_{i}}-\frac{2}{3} \eta^{i j} \frac{\partial P^{l}}{\partial x^{l}}\right)+\zeta \frac{1}{\rho_{0}+p_{0}} \frac{\partial}{\partial x_{i}} \frac{\partial P^{l}}{\partial x^{l}} .
\end{aligned}
$$

We note that there appear the relativistic corrections with the time derivative. If those terms are dropped, the dissipation terms reduce to the non-relativistic ones. Moreover we note that the thermal conductivity $\lambda$ enters into the equation for $P^{i}$ as a relativistic effect.

When moving to the Fourier space, we finally obtain

$$
\begin{aligned}
\frac{\partial E_{\vec{q}}}{\partial t}= & -\mathrm{i} q_{j} P_{\vec{q}}^{j}+\lambda\left(-\frac{1}{C} q^{2} E_{\vec{q}}+\frac{T_{0}}{\rho_{0}+p_{0}} \mathrm{i} q_{j} \frac{\partial P_{\vec{q}}^{j}}{\partial t}\right) \\
\frac{\partial P_{\vec{q}}^{i}}{\partial t}= & -\mathrm{i} q_{j} T_{0 \vec{q}}^{i j}+\lambda\left(\frac{1}{C} \mathrm{i} q^{i} \frac{\partial E_{\vec{q}}}{\partial t}+\frac{T_{0}}{\rho_{0}+p_{0}} \frac{\partial^{2} P_{\vec{q}}^{i}}{\partial t^{2}}\right) \\
& +\frac{\eta}{\rho_{0}+p_{0}} \mathrm{i} q_{j}\left(\mathrm{i} q^{j} P_{\vec{q}}^{i}+\mathrm{i} q^{i} P_{\vec{q}}^{j}-\frac{2}{3} \eta^{i j} \mathrm{i} q_{l} P_{\vec{q}}^{l}\right)+\frac{\zeta}{\rho_{0}+p_{0}} \mathrm{i} q^{i} \mathrm{i} q_{l} P_{\vec{q}}^{l},
\end{aligned}
$$


from which we find the dissipation terms in Eqs. (5.48), (5.55).

\section{Scaling properties of the commutators of the slow variables}

In this appendix, we examine the scaling properties of the commutators of the fluctuating amplitudes, $\left[A_{j}, A_{l}\right]$ for $\left\{\phi, \phi^{\dagger}, Q, E, P^{i}\right\}$ in the chiral phase transition. The results provide us with the exponents $x_{j l}$, which are used to find the scaling properties of the commutators of the reduced amplitudes, $\left[\tilde{A}_{j}, \tilde{A}_{l}\right]$.

- $\left[\phi_{\alpha \vec{q}},\left(\phi_{\alpha \vec{q}^{\prime}}\right)^{\dagger}\right]$

The commutation relation is given in Eq.(A.1).

$$
\left[\phi_{\alpha \vec{q}},\left(\phi_{\alpha^{\prime} \vec{q}^{\prime}}\right)^{\dagger}\right]=\frac{1}{2 \omega_{\vec{q}}} \delta_{\alpha \alpha^{\prime}} \delta_{\vec{q} \vec{q}^{\prime}} \sim \xi^{\frac{\gamma}{2 \nu}}
$$

where we have used $\omega_{\vec{q}}=\left(2 \chi_{\phi \vec{q}}\right)^{-1 / 2} \sim \xi^{-\gamma / 2 \nu}$.

- $\left[\phi_{\alpha \vec{q}},\left(\phi_{\alpha^{\prime}-\vec{q}^{\prime}}^{\dagger}\right)^{\dagger}\right]$

This is zero.

- $\left[\phi_{\alpha \vec{q}},\left(Q_{\alpha^{\prime} \beta^{\prime} \vec{q}^{\prime}}\right)^{\dagger}\right]$

The commutation relation is given in Eq. (A.3). Noting that

$$
V^{-\frac{1}{2}}=\left(\frac{V}{\xi^{d}} \xi^{d}\right)^{-\frac{1}{2}} \sim \xi^{-\frac{d}{2}}
$$

we find

$$
\begin{aligned}
{\left[\phi_{\alpha \vec{q}},\left(Q_{\alpha^{\prime} \beta^{\prime} \vec{q}^{\prime}}\right)^{\dagger}\right] } & =\frac{-\mathrm{i}}{2 \sqrt{V}} \epsilon_{\alpha \alpha^{\prime} \beta^{\prime} \gamma}\left\{\phi_{\gamma \vec{q}-\vec{q}^{\prime}}+\phi_{\gamma \vec{q}^{\prime}-\vec{q}}^{\dagger}+\frac{\omega_{\vec{q}-\vec{q}^{\prime}}}{\omega_{\vec{q}}}\left(\phi_{\gamma \vec{q}-\vec{q}^{\prime}}-\phi_{\gamma \vec{q}^{\prime}-\vec{q}}^{\dagger}\right)\right\} \\
& \sim \xi^{-\frac{d}{2}+\frac{\gamma}{2 \nu}} .
\end{aligned}
$$

- $\left[\phi_{\alpha \vec{q}},\left(E_{\vec{q}^{\prime}}\right)^{\dagger}\right]$

Using the approximation $\left[A_{\vec{k}}, B_{\vec{k}^{\prime}}\right] \sim\left[A_{\vec{k}+\vec{l}}, B_{\vec{k}^{\prime}-\vec{l}}\right]$ with $|\vec{k}|,\left|\overrightarrow{k^{\prime}}\right|,|\vec{l}| \ll 1$, we find

$$
\left[\phi_{\alpha \vec{q}}, E_{\vec{q}^{\prime}}^{\dagger}\right]=\left[\phi_{\alpha \vec{q}}, E_{-\vec{q}^{\prime}}\right] \sim\left[\phi_{\alpha \vec{q}-\vec{q}^{\prime}}, E_{\overrightarrow{0}}\right]=\left[\phi_{\alpha \vec{q}-\vec{q}^{\prime}}, \frac{1}{\sqrt{V}} \mathcal{H}\right]=\frac{\mathrm{i}}{\sqrt{V}} \dot{\phi}_{\alpha \vec{q}-\vec{q}^{\prime}}
$$

namely

$$
\left[\phi_{\alpha \vec{q}}, E_{\vec{q}^{\prime}}^{\dagger}\right]=\frac{\mathrm{i}}{\sqrt{V}} \dot{\phi}_{\alpha}\left(\vec{q}-\vec{q}^{\prime}, \xi, V\right)
$$


The scaling property of $\dot{\phi}$ can be found by considering

$$
\begin{aligned}
& \left(\mathrm{i} \dot{\phi}_{\alpha \vec{k}}, \phi_{\beta-\vec{k}^{\prime}}^{\dagger} Q_{\alpha^{\prime} \beta^{\prime}-\vec{k}-\vec{k}^{\prime}}\right)=k_{\mathrm{B}} T\left\langle\left[\phi_{\alpha \vec{k}}, \phi_{\beta-\vec{k}^{\prime}}^{\dagger} Q_{\alpha^{\prime} \beta^{\prime}-\vec{k}-\vec{k}^{\prime}}\right]\right\rangle \\
& \quad=\frac{-\mathrm{i}}{2 \sqrt{V}} k_{\mathrm{B}} T\left\langle\phi_{\beta-\vec{k}}^{\dagger} \epsilon_{\alpha \alpha^{\prime} \beta^{\prime} \gamma}\left\{\phi_{\gamma-\vec{k}^{\prime}}+\phi_{\gamma \vec{k}^{\prime}}^{\dagger}+\frac{\omega_{\vec{k}^{\prime}}}{\omega_{\vec{k}}}\left(\phi_{\gamma-\vec{k}^{\prime}}-\phi_{\gamma \vec{k}^{\prime}}^{\dagger}\right)\right\}\right\rangle .
\end{aligned}
$$

Denoting the exponent of $\dot{\phi}$ as $x_{\dot{\phi}}$, we find

$$
L^{\frac{3}{2} d-x_{\phi}-x_{\phi}-x_{Q}}=L^{-\frac{d}{2}} L^{d-2 x_{\phi}},
$$

which gives $x_{\dot{\phi}}=d / 2+\beta / \nu$. Thus $\dot{\phi}$ scales as

$$
\dot{\phi}(\vec{k}, \xi, V)=L^{\frac{d}{2}-x_{\dot{\phi}}} \dot{\phi}\left(L \vec{k}, \xi / L, V / L^{d}\right) \sim \xi^{-\frac{\beta}{\nu}},
$$

leading to

$$
\left[\phi_{\alpha \vec{q}},\left(E_{\vec{q}}\right)^{\dagger}\right] \sim \xi^{-\frac{d}{2}-\frac{\beta}{\nu}}
$$

- $\left[\phi_{\alpha \vec{q}},\left(P_{\vec{q}^{\prime}}^{i}\right)^{\dagger}\right]$

From Eq. (A.5),

$$
\left[\phi_{\alpha \vec{q}},\left(P_{\vec{q}^{\prime}}^{i}\right)^{\dagger}\right]=\frac{1}{2 \sqrt{V}}\left\{\left(q-q^{\prime}\right)^{i}\left(\phi_{\alpha \vec{q}-\vec{q}^{\prime}}+\phi_{\alpha \vec{q}^{\prime}-\vec{q}}^{\dagger}\right)+\frac{\omega_{\vec{q}-\vec{q}^{\prime}}}{\omega_{\vec{q}}} q^{i}\left(\phi_{\alpha \vec{q}-\vec{q}^{\prime}}-\phi_{\alpha \vec{q}^{\prime}-\vec{q}}^{\dagger}\right)\right\} .
$$

This gives the scaling properties,

$$
\left[\phi_{\alpha \vec{q}},\left(P_{\vec{q}^{\prime}}^{\mathrm{L} i}\right)^{\dagger}\right] \sim\left[\phi_{\alpha \vec{q}},\left(P_{\vec{q}^{\prime}}^{T i}\right)^{\dagger}\right] \sim \xi^{-\frac{d}{2}-1+\frac{\gamma}{2 \nu}}
$$

for the longitudinal and transverse components of the momentum.

- $\left[Q_{\alpha \beta \vec{q}},\left(Q_{\alpha^{\prime} \beta^{\prime} \vec{q}^{\prime}}\right)^{\dagger}\right]$

The commutation relation Eq. (A.10) immediately gives

$$
\left[Q_{a \vec{q}}^{V},\left(Q_{b \vec{q}^{\prime}}^{V}\right)^{\dagger}\right]=\frac{\mathrm{i}}{\sqrt{V}} \epsilon_{a b c} Q_{c \vec{q}-\vec{q}^{\prime}}^{V} \sim \xi^{-\frac{d}{2}} .
$$

- $\left[Q_{\alpha \beta \vec{q}},\left(E_{\vec{q}}\right)^{\dagger}\right]$

As before, the commutator is approximated as

$$
\left[Q_{\alpha \beta \vec{q}}, E_{-\vec{q}^{\prime}}\right] \sim\left[Q_{\alpha \beta \vec{q}-\vec{q}^{\prime}}, E_{\overrightarrow{0}}\right]=\left[Q_{\alpha \beta \vec{q}-\vec{q}^{*}}, \frac{1}{\sqrt{V}} \mathcal{H}\right]=\frac{\mathrm{i}}{\sqrt{V}} \dot{Q}_{\alpha \beta \vec{q}-\vec{q}^{*}},
$$

that is,

$$
\left[Q_{\alpha \beta \vec{q}}, E_{-\vec{q}^{\prime}}\right]=\frac{\mathrm{i}}{\sqrt{V}} \dot{Q}_{\alpha \beta}\left(\vec{q}-\vec{q}^{\prime}, \xi, V\right) .
$$


Consider

$$
\begin{aligned}
\left(\dot{\mathrm{Q}}_{\alpha \beta \vec{k}}, Q_{\alpha^{\prime} \beta^{\prime} \vec{k}^{\prime}} Q_{\gamma^{\prime} \delta^{\prime}-\vec{k}-\vec{k}^{\prime}}\right) & =k_{\mathrm{B}} T\left\langle\left[Q_{\alpha \beta \vec{k}}, Q_{\alpha^{\prime} \beta^{\prime} \vec{k}^{\prime}} Q_{\gamma^{\prime} \beta^{\prime}-\vec{k}-\vec{k}^{\prime}}\right]\right\rangle \\
& \sim k_{\mathrm{B}} T\left\langle\frac{i}{\sqrt{V}} Q_{\vec{k}+\vec{k}^{\prime}} Q_{-\vec{k}-\vec{k}^{\prime}}+\frac{i}{\sqrt{V}} Q_{\vec{k}^{\prime}} Q_{-\vec{k}^{\prime}}\right\rangle .
\end{aligned}
$$

This gives

$$
L^{\frac{3}{2} d-x_{\dot{Q}}-2 x_{Q}}=L^{-\frac{d}{2}} L^{d-2 x_{Q}}
$$

to find $x_{\dot{Q}}=d$ where $x_{\dot{Q}}$ denotes the exponent for $\dot{Q}$. The scaling property of $\dot{Q}$ is thus

$$
\dot{Q}_{\alpha \beta}(\vec{k}, \xi, V)=L^{\frac{d}{2}-x_{\dot{Q}}} \dot{Q}_{\alpha \beta}\left(L \vec{k}, \xi / L, V / L^{d}\right) \sim \xi^{-\frac{d}{2}},
$$

from which we obtain

$$
\left[Q_{\alpha \beta \vec{q}}, E_{-\vec{q}^{\prime}}\right]=\frac{\mathrm{i}}{\sqrt{V}} \dot{Q}_{\alpha \beta}\left(\vec{q}-\vec{q}^{\prime}, \xi, V\right) \sim \xi^{-d} .
$$

- $\left[Q_{\alpha \beta \vec{q}},\left(P_{\vec{q}^{\prime}}^{i}\right)^{\dagger}\right]$

The commutation relation Eq. (A.14) gives

$$
\left[Q_{\alpha \beta \vec{q}},\left(P_{\vec{q}^{\prime}}^{\mathrm{L} i}\right)^{\dagger}\right] \sim\left[Q_{\alpha \beta \vec{q}},\left(P_{\vec{q}^{\prime}}^{\mathrm{T} i}\right)^{\dagger}\right] \sim \xi^{-\frac{d}{2}-1}
$$

- $\left[E_{\vec{q}},\left(E_{\vec{q}^{\prime}}\right)^{\dagger}\right]$

The commutation relation Eq. (A.15) gives

$$
\left[E_{\vec{q}},\left(E_{\vec{q}^{\prime}}\right)^{\dagger}\right] \sim \xi^{-\frac{d}{2}-1} .
$$

- $\left[E_{\vec{q}},\left(P_{\vec{q}^{\prime}}^{i}\right)^{\dagger}\right]$

The same procedure as before leads us to

$$
\left[E_{\vec{q}},\left(P_{\vec{q}^{\prime}}^{i}\right)^{\dagger}\right] \sim\left[E_{\overrightarrow{0}}, P_{\vec{q}-\vec{q}^{\prime}}^{i}\right]=\frac{-\mathrm{i}}{\sqrt{V}} \dot{P}_{\vec{q}-\vec{q}^{\prime}}^{i},
$$

that is,

$$
\left[E_{\vec{q}}, P_{-\vec{q}^{\prime}}^{i}\right]=\frac{-\mathrm{i}}{\sqrt{V}} \dot{P}^{i}\left(\vec{q}-\vec{q}^{\prime}, \xi, V\right)
$$

Consider

$$
\begin{aligned}
& \left(\mathrm{i} \dot{P}_{\vec{k}}^{i}, P_{\vec{k}^{\prime}}^{j} P_{-\vec{k}-\vec{k}^{\prime}}^{l}\right)=k_{\mathrm{B}} T\left\langle\left[P_{\vec{k}}^{i}, P_{\vec{k}^{\prime}}^{j} P_{-\vec{k}-\vec{k}^{\prime}}^{l}\right]\right\rangle \\
& =\frac{k_{\mathrm{B}} T}{\sqrt{V}}\left\{k^{j}\left\langle P_{\vec{k}+\vec{k}^{\prime}}^{i} P_{-\vec{k}-\vec{k}^{\prime}}^{l}\right\rangle-k^{i}\left\langle P_{\vec{k}+\vec{k}^{\prime}}^{j} P_{-\vec{k}-\vec{k}^{\prime}}^{l}\right\rangle\right. \\
& \left.+k^{l}\left\langle P_{\vec{k}^{\prime}}^{j} P_{-\vec{k}^{\prime}}^{i}\right\rangle+\left(k+k^{\prime}\right)^{i}\left\langle P_{\vec{k}^{\prime}}^{j} P_{-\vec{k}^{\prime}}^{l}\right\rangle\right\} \text {, }
\end{aligned}
$$


which gives the exponent for $\dot{P}$ as $x_{\dot{P}}=d+1$. Thus

$$
\dot{P}^{i}(\vec{k}, \xi, V)=L^{\frac{d}{2}-x} \dot{P} \dot{P}^{i}\left(L \vec{k}, \xi / L, V / L^{d}\right) \sim \xi^{-\frac{d}{2}-1},
$$

and we find

$$
\left[E_{\vec{q}},\left(P_{\vec{q}^{\prime}}^{i}\right)^{\dagger}\right]=\frac{-\mathrm{i}}{\sqrt{V}} \dot{P}^{i}\left(\vec{q}-\vec{q}^{\prime}, \xi, V\right) \sim \xi^{-d-1}
$$

- $\left[P_{\vec{q}}^{i},\left(P_{\vec{q}^{\prime}}^{j}\right)^{\dagger}\right]$

The commutation relation Eq. (A.17) gives

$$
\left[P_{\vec{q}}^{i},\left(P_{\vec{q}^{\prime}}^{j}\right)^{\dagger}\right] \sim \xi^{-\frac{d}{2}-1}
$$

\section{References}

[1] R. D. Pisarski and F. Wilczek, Phys. Rev. D29, (1984) 338; F. Wilczek, Int. J. Mod. Phys. A7, (1992) 3911 [Erratum-ibid. A7, (1992) 6951].

[2] F. Karsch and E. Laermann, Phys. Rev. D50, (1994) 6954 arXiv:hep-lat/9406008.

[3] S. Aoki et al. [JLQCD Collaboration], Phys. Rev. D57, (1998) 3910 arXiv:hep-lat/9710048.

[4] C. W. Bernard et al., Phys. Rev. D61, (2000) 054503 arXiv:hep-lat/9908008.

[5] E. Laermann and O. Philipsen, arXiv:hep-ph/0303042.

[6] T. Hatsuda and T. Kunihiro, Phys. Rev. Lett. 55 (1985) 158; Prog. Theor. Phys. 74 (1985) 765.

[7] K. Rajagopal and F. Wilczek, Nucl. Phys. B399 (1993) 395 arXiv:hep-ph/9210253.

[8] D. T. Son and M. A. Stephanov, Phys. Rev. Lett. 88, (2002) 202302 arXiv:hep-ph/0111100; Phys. Rev. D66, (2002) 076011 arXiv:hep-ph/0204226.

[9] D. Boyanovsky and H. J. De Vega, Phys. Rev. D65 (2002) 085038 arXiv:hep-ph/0110012; Annals Phys. 307 (2003) 335 arXiv:hep-ph/0302055.

[10] T. Koide and M. Maruyama, arXiv:nucl-th/0308025; arXiv:hep-ph/0404133

[11] J. P. Blaizot and E. Iancu, Phys. Rept. 359 (2002) 355 arXiv:hep-ph/0101103.

[12] J. Berges and J. Serreau, arXiv:hep-ph/0302210.

[13] T. Ikeda, Phys. Rev. D69, (2004) 105018 arXiv:hep-ph/0401045. 
[14] P. C. Hohenberg and B. I. Halperin, Rev. Mod. Phys. 49 (1977) 435.

[15] K. Kawasaki, Prog. Theor. Phys. 39 (1968) 1133; ibid. 40 (1968) 11; ibid. 40 (1968) 706.

[16] K. Kawasaki, Ann. Phys. 61 (1970) 1.

[17] K. Kawasaki, in Phase Transitions and Critical Phenomena, edited by C. Domb and M.S. Green, Vol. 5a, (Academic, New York) (1976).

[18] K. Kawasaki, Non-Equilibrium and Phase Transition, in Japanese, Asakurashoten (2000).

[19] P. C. Hohenberg and B. I. Halperin, Phys. Rev. 177 (1969) 952.

[20] P. C. Hohenberg, B. I. Halperin and E. D. Siggia, Phys. Rev. B13 (1976) 1299.

[21] H. Mori, Prog. Theor. Phys. 33 (1965) 423.

[22] R. Zwanzig, J. Chem. Phys. 33 (1960) 1338.

[23] M. Fixman, J. Chem. Phys. 36 (1962) 310.

[24] L. van Hove, Phys. Rev. 93 (1954) 1374.

[25] L. P. Kadanoff and P. C. Martin, Ann. Phys. 24 (1963) 419.

[26] D. Forster, Hydrodynamic Fluctuations, Broken Symmetry, and Correlation Functions, W. A. Benjamin (1975).

[27] P. C. Hohenberg and P. C. Martin, Ann. Phys. 34 (1965) 291.

[28] G. Baker, B. Nickel and D. Meiron, Phys. Rev. B17 (1978) 1365.

[29] M. Asakawa and K. Yazaki, Nucl. Phys. A504 (1989) 668.

[30] J. Berges and K. Rajagopal, Nucl. Phys. B538 (1999) 215 arXiv:hep-ph/9804233.

[31] M. A. Halasz, A. D. Jackson, R. E. Shrock, M. A. Stephanov and J. J. M. Verbaarschot, Phys. Rev. D58 (1998) 096007 arXiv:hep-ph/9804290.

[32] M. A. Stephanov, K. Rajagopal and E. V. Shuryak, Phys. Rev. Lett. 81 (1998) 4816 arXiv:hep-ph/9806219; Phys. Rev. D60 (1999) 114028 arXiv:hep-ph/9903292.

[33] K. Fukushima, Phys. Rev. C67 (2003) 025203 arXiv:hep-ph/0209270.

[34] H. Fujii, Phys. Rev. D67 (2003) 094018 arXiv:hep-ph/0302167; H. Fujii and M. Ohtani, arXiv:hep-ph/0402263.

[35] D. T. Son and M. A. Stephanov, arXiv:hep-ph/0401052.

[36] S. Weinberg, Gravitation and Cosmology, John Wiley \& Sons (1972). 